\title{
Cq-ROFRS: covering q-rung orthopair fuzzy rough sets and its application to multi-attribute decision-making process
}

\author{
Harish Garg $^{1}$ (D) $\cdot$ Mohammed Atef ${ }^{2}$
}

Received: 13 July 2021 / Accepted: 10 December 2021 / Published online: 2 February 2022

(C) The Author(s) 2022

\begin{abstract}
Pythagorean fuzzy sets (briefly, PFSs) were created as an upgrade to intuitionistic fuzzy sets (briefly, IFSs) which helped to address some problems that IFSs couldn't solve. The definition of q-rung orthopair fuzzy sets (briefly, q-ROFS) is then declared to generalize and solve PFS and IFS failures. Using the concept of PF $\beta$-neighborhood, Zhan et al. defined the description of the covering through the Pythagorean fuzzy rough set (briefly, CPFRS). Hussain et al. also developed the concept of q-ROF $\beta$-neighborhood to build the concept of covering through q-rung orthopair fuzzy rough sets (Cq-ROFRS). To enhance the results in Zhan et al.'s and Hussain et al.'s method and in a related context, the concept of PF complementary $\beta$-neighborhood is constructed. Hence, using PF $\beta$-neighborhood and PF complementary $\beta$-neighborhood, three novel kinds of CPFRS are investigated and the related characteristics are analyzed. The interrelationships between Zhan et al.'s approach and our approaches are also discussed. Besides, the concept of q-ROF complementary $\beta$-neighborhood is examined. Three new Cq-ROFRS models are differentiated using the principles of q-ROF $\beta$-neighborhood and q-ROF complementary $\beta$ neighborhood. As a result, the related properties and relationships between these various models and Hussain et al.'s model are established. Because of these correlations, we may consider our approach to be a generalization of Zhan et al.'s and Hussain et al's approaches. Finally, we developed applications to solve MADM problems using CPFRS and Cq-ROFRS, as well as variances of the two methods using numerical examples are presented.
\end{abstract}

Keywords PF $\beta$-neighborhoods $\cdot$ CPFRS $\cdot$ q-ROF $\beta$-neighborhoods $\cdot$ Cq-ROFRS $\cdot$ MAPFDM $\cdot$ MAq-ROFDM

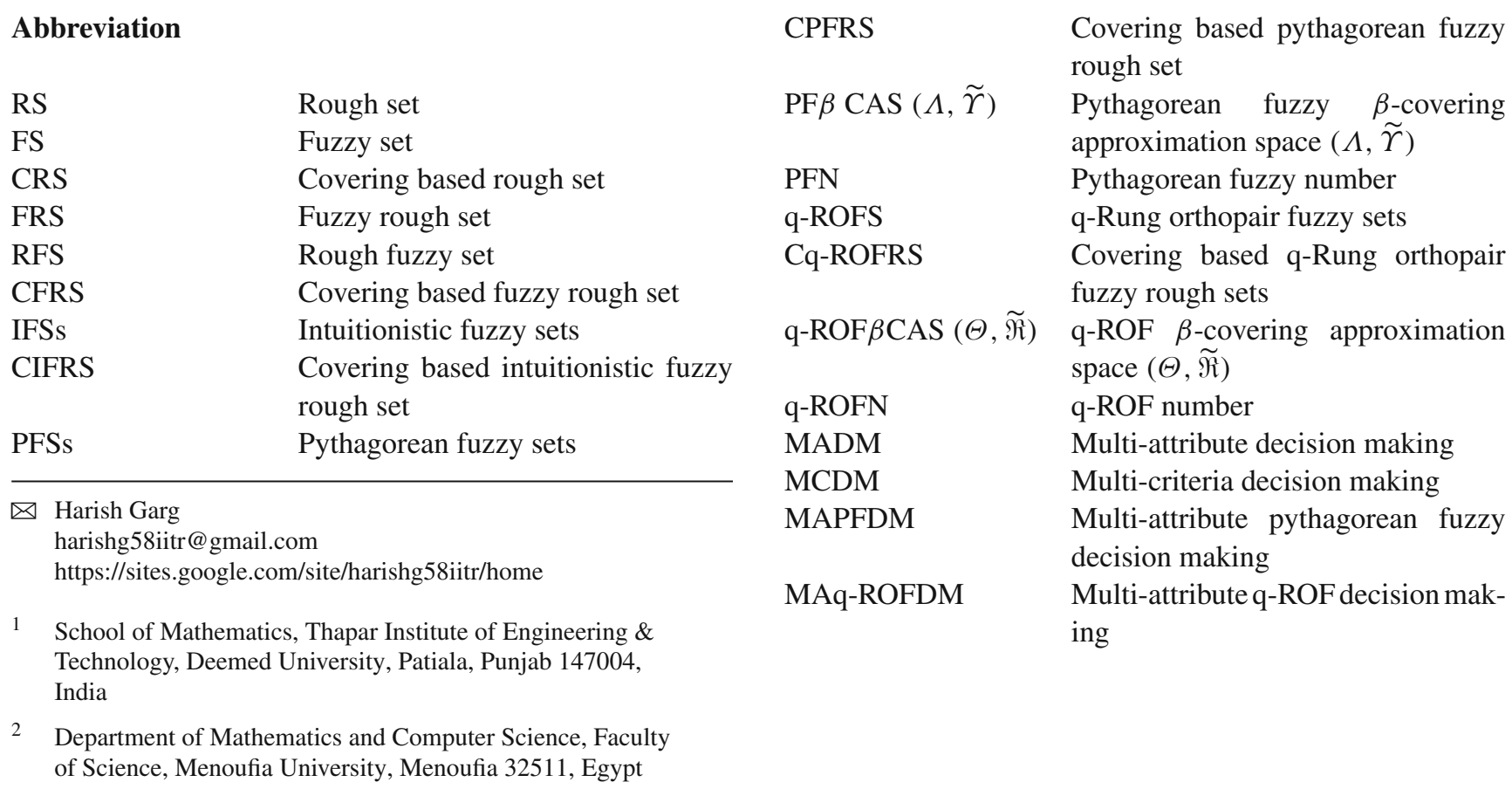




\section{Introduction}

Rough set (RS) theory was established for adapting the ambiguity and granularity in data via Pawlak $[1,2]$. It is also utilized in various areas such as neighborhood systems, graphs, kernel, reduction, granulation, probabilistic rough set, variable precision and so on [3-13]. The most important generalization of this theory is covering via rough set (CRS). Many researchers worked on CRS as Pomykala $[14,15]$ studied the two pairs of dual operator, Yao $[16,17]$ extent these operators via the neighborhood and granularity, Couso et al. [18] construct CRS with incomplete data, Bonikowski et al. [19] established CRS through minimal description, Zhu [20] discussed topological properties to CRS, Zhu et al. [21] presented the reduction on CRS, Zhu et al. [22,23], Tsang et al. [24] and Xu et al. [25] introduced other kinds of CRS, Liu et al. [26] built the differences between CRS Zhu's and Xu's models, Ma [27] investigated some kinds of neighborhoods via CRS

The concept of fuzzy rough set (FRS) and rough fuzzy set (RFS) was constructed by Dubois et al. [28]. Deng et al. [29] proposed new model of fuzzy covering according to FRS. Atef et al. and $\mathrm{Li}$ et al. studied additional kinds of fuzzy rough covering (FRC) [30-34]. Also, Ma [35] discovered kinds of fuzzy covering rough set (FCRS) using the fuzzy $\beta$-neighborhood. Moreover, the notions of a fuzzy complementary $\beta$-neighborhood and fuzzy $\beta$ minimal and maximal description were found by Yang et al. [36,37].

Fuzzy set theory (FS) was initiated by Zadeh [38]. There are some problems in FS for dealing with uncertain data, so the definition of IFSs was appeared by Atanassov [39] which contain two parts membership degree and non-membership degree. In IFSs, the sum of membership and non-membership classes is between [0, 1]. Atanassov et al. [40] used IFS to make a decision multi-person problem. Huang et al. $[41,42]$ introduced the notion of intuitionistic fuzzy multigranulation rough sets and intuitionistic fuzzy via CRS. Alcantud et al. [43] discussed the decomposition theorems.

In realistic problems, much difficult application can not be solved via IFSs. Therefore, Yager [44] introduced the concept of PFSs. The main adding in PFSs is the sum of squares of membership class and non-membership class is in $[0,1]$. Yager $[45,46]$ suggested the usage of PFSs to solve real problem and make a decision. Garg $[47,48]$ studied the generalized Pythagorean fuzzy information aggregation using Einstein operations and Pythagorean geometric aggregation operations using Einstein t-norm with their applications. Zhang et al. [49] extend the PFSs to solve MCDM issues. Hussain et al. [50] defined the concept of Pythagorean fuzzy soft rough sets. Wang and Garg [51] introduced an algorithm for MADM by Pythagorean fuzzy archimedean norm operations. Recently, Zhan et al. [52] established the definition of CPFRS according to a PF $\beta$-neighborhood. They used these notions to solve problems in multi-attribute Pythagorean fuzzy decision making (MAPFDM).

From profounding in the real application, there were some problems not solved by IFSs and PFSs. So, In 2017, Yager [53] demonstrate a new notion to solve these issues in IFSs and PFSs. This notion called the q-rung orthopair fuzzy sets (q-ROFSs) are considered a generalization of PFSs and IFSs. The sum of qth power of membership class and $q$ th power of nonmembership class is in the interval $[0,1]$ in q-ROFSs. In recent years, q-ROFSs studied and applied more widely in many distinct areas. Yager and Alajlan [54] discussed the relevant characteristics of q-ROFSs. In 2019, there was a new think of q-ROFSs via orbits by Ali [55]. The notions of connection number based q-ROFS is developed by Garg [56]. Especially, Hussain et al. [57] introduced the definition of CqROFRS through the notion of $\mathrm{q}-\mathrm{ROF} \beta$-neighborhood and applied it in multi-attribute q-ROF decision making (MAqROFDM). These differences illustrate that $3-\mathrm{PF} \beta C R S$ is the best approximations among 1-PF $\beta$ CRS (Zhan et al.'s model), 2-PF $\beta$ CRS and 4-PF $\beta$ CRS.

The main aim of this study is to improve Zhan et al.'s [52] and Hussain et al.'s [57] studies, by overgrowing the lower approximation and diminish the upper approximation of the proposed methods. Thus, we set the meaning of PF complementary $\beta$-neighborhood and hence we present a new type of CPFRS model (2-PF $\beta C R S$ ). To generalize this study, we obtain two new PF $\beta$-neighborhoods by joining $\mathrm{PF} \beta$-neighborhood and PF complementary $\beta$-neighborhood and then two new CPFRS models are built (3-PF $\beta$ CRS and 4-PF $\beta C R S$ ). The properties of these models are also discussed. Further, the relationships through the Zhan et al.'s model and our models (i.e., 1-PF $\beta$ CRS, 2-PF $\beta$ CRS, 3 -PF $\beta$ CRS and 4-PF $\beta C R S$ ) are investigated. Moreover, we put forward the definition of q-ROF complementary $\beta$ neighborhood and using it to introduce a novel model of Cq-ROFRS (2-q-ROF $\beta$ CRS). Hence, we merge the definitions of $\mathrm{q}-\mathrm{ROF} \beta$-neighborhood and $\mathrm{q}-\mathrm{ROF}$ complementary $\beta$-neighborhood to generate two new kinds of q-ROF $\beta$ neighborhood (3-q-ROF $\beta$ CRS and 4-q-ROF $\beta$ CRS). We use these kinds to give two other paradigms of Cq-ROFRS and also study relevant properties. Relationships between Hussain et al.'s model and our's (i.e., 1-q-ROF $\beta$ CRS, 2$\mathrm{q}-\mathrm{ROF} \beta \mathrm{CRS}, 3-\mathrm{q}-\mathrm{ROF} \beta \mathrm{CRS}$ and 4-q-ROF $\beta$ CRS) are also given. We put forward some examples to explain the differences between these two approaches which conclude that 3-PF $\beta$ CRS is the best among others (i.e., 1-PF $\beta$ CRS, $2-\mathrm{PF} \beta \mathrm{CRS}$ and $4-\mathrm{PF} \beta \mathrm{CRS}$ ) and 3 -q-ROF $\beta$ CRS is more accurate than others (i.e., 1-q-ROF $\beta$ CRS, 2-q-ROF $\beta$ CRS and $4-\mathrm{q}-\mathrm{ROF} \beta \mathrm{CRS}$ ). Finally, we apply the presented work to solve MAPFDM and MAq-ROFDM problems.

The rest of the article is as follows. The basic notions about PFSs and q-ROFSs are set in "Preliminaries". "PF complementary $\beta$-neighborhood and three novel kinds of 
CPFRS" constructs three new models of CPFRS by means of PF $\beta$-neighborhood and PF complementary $\beta$-neighborhood. We determine the definition of q-ROF complementary $\beta$ neighborhood and use it to build three models of Cq-ROFRS with the help of $\mathrm{q}$-ROF $\beta$-neighborhood in " $\mathrm{q}-\mathrm{ROF}$ complementary $\beta$-neighborhood and three novel kinds of CqROFRS". In "Decision-making approach using PF $\beta$ CAS", we give numerical examples via our methods to explain the theoretical studies. We put forward the main goals of this study in "Conclusion".

\section{Preliminaries}

In the following, we supply a short scanning of some concepts consumed over the paper.

Definition 1 [49] Let $\Lambda$ be a universe. For every $u \in \Lambda$, if we have a membership function $\vartheta_{\mathcal{P}}: \Lambda \rightarrow[0,1]$ and a nonmembership function $\zeta_{\mathcal{P}}: \Lambda \rightarrow[0,1]$. Define the PFS $\mathcal{P}$ as indicated below.

$$
\mathcal{P}=\left\{\left(u, \vartheta_{\mathcal{P}}(u), \zeta_{\mathcal{P}}(u)\right)\right\},
$$

where $0 \leq \vartheta_{\mathcal{P}}^{2}(u)+\zeta_{\mathcal{P}}^{2}(u) \leq 1$.

Definition 2 [49] Consider a PFS $\mathcal{P} \in \Lambda$, define the grade of indeterminacy of $u \in \Lambda$ to $\mathcal{P}$ as follows.

$\xi_{\mathcal{P}}(u)=\sqrt{1-\vartheta_{\mathcal{P}}^{2}(u)-\zeta_{\mathcal{P}}^{2}(u)}$.

Definition 3 [49] Let $\mathcal{P}_{1}=\left(\vartheta_{\mathcal{P}_{1}}, \zeta_{\mathcal{P}_{1}}\right)$ and $\mathcal{P}_{2}=\left(\vartheta_{\mathcal{P}_{2}}, \zeta_{\mathcal{P}_{2}}\right)$, for $\mathcal{P}_{1}, \mathcal{P}_{2} \in \operatorname{PF}(\Lambda)$. Then $\forall u \in \Lambda$, we have the following properties

(1) $\mathcal{P}_{1}^{c}=\left\{\left(u, \zeta \mathcal{P}_{1}(u), \vartheta_{\mathcal{P}_{1}}(u)\right)\right\}$.

(2) $\mathcal{P}_{1}=\mathcal{P}_{2} \Longleftrightarrow \vartheta_{\mathcal{P}_{1}}(u)=\vartheta_{\mathcal{P}_{2}}(u)$ and $\zeta \mathcal{P}_{1}(u)=$ $\zeta \mathcal{P}_{2}(u)$.

(3) $\mathcal{P}_{1} \subseteq \mathcal{P}_{2}$ if $\vartheta_{\mathcal{P}_{1}}(u) \leq \vartheta_{\mathcal{P}_{2}}(u)$ and $\zeta_{\mathcal{P}_{1}}(u) \leq \zeta \mathcal{P}_{2}(u)$.

(4) $\mathcal{P}_{1} \cap \mathcal{P}_{2}=\left\{\left(u, \vartheta_{\mathcal{P}_{1}}(u) \wedge \vartheta_{\mathcal{P}_{2}}(u), \zeta_{\mathcal{P}_{1}}(u) \vee \zeta_{\mathcal{P}_{2}}(u)\right)\right\}$.

(5) $\mathcal{P}_{1} \cup \mathcal{P}_{2}=\left\{\left(u, \vartheta_{\mathcal{P}_{1}}(u) \vee \vartheta_{\mathcal{P}_{2}}(u), \zeta \mathcal{P}_{1}(u) \wedge \zeta \mathcal{P}_{2}(u)\right)\right\}$.

(6) $\mathcal{P}_{1}-\mathcal{P}_{2}=\mathcal{P}_{1} \cap \mathcal{P}_{2}^{c}$.

Definition 4 [49] Let $\mathcal{P}_{1}=\left(\vartheta_{\mathcal{P}_{1}}, \zeta_{\mathcal{P}_{1}}\right)$ and $\mathcal{P}_{2}=\left(\vartheta_{\mathcal{P}_{2}}, \zeta_{\mathcal{P}_{2}}\right)$ be two PFNs. Then the distances among them is defined as follows.

$\mathcal{E}\left(\mathcal{P}_{1}, \mathcal{P}_{2}\right)=\frac{1}{2}\left[\left|\vartheta_{\mathcal{P}_{1}}^{2}-\vartheta_{\mathcal{P}_{2}}^{2}\right|+\left|\zeta_{\mathcal{P}_{1}}^{2}-\zeta_{\mathcal{P}_{2}}^{2}\right|+\left|\xi_{\mathcal{P}_{1}}^{2}-\xi_{\mathcal{P}_{2}}^{2}\right|\right]$.

Zhan et al. [52] introduced the notion of CPFRS and put forward the definition of PF $\beta$-neighborhood as indicated below.
Definition 5 [52] Consider $\Lambda$ be a universe and $\tilde{\Upsilon}=$ $\left\{\widetilde{\Delta}_{1}, \widetilde{\Delta}_{2}, \ldots, \widetilde{\Delta}_{m}\right\}$ where $\widetilde{\Delta}_{i} \in \operatorname{PF}(\Lambda)$ and $i=1,2, \ldots, m$. Then for each PFN $\beta=\left(\vartheta_{\beta}, \zeta_{\beta}\right)$, a Pythagorean fuzzy $\beta$ covering of $\Lambda$, if $\widetilde{\Delta}_{i}(u) \geq \beta$ for each $u \in \Omega$. Now $(\Lambda, \widetilde{\Upsilon})$ is called the Pythagorean fuzzy $\beta$-covering approximation space (briefly, PF $\beta$ CAS).

Definition 6 [52] Consider a $\operatorname{PF} \beta \operatorname{CAS}(\Lambda, \tilde{\Upsilon})$, for some PFN $\beta=\left(\vartheta_{\beta}, \zeta_{\beta}\right)$. For every $u \in \Lambda$, define the PF $\beta$ neighborhood of $u$ as follows.

$\widetilde{\mathcal{P}}_{u}^{\beta}=\cap\left\{\widetilde{\Delta}_{i} \in \tilde{\Upsilon}: \widetilde{\Delta}_{i}(u) \geq \beta\right\}$.

Definition 7 [52] Consider a $\operatorname{PF} \beta \operatorname{CAS}(\Lambda, \tilde{\Upsilon})$, for some PFN $\beta=\left(\vartheta_{\beta}, \zeta_{\beta}\right)$. For every $u \in \Lambda$ and $\mathcal{X} \in P F(\Lambda)$, that is, $\mathcal{X}=\left\{\left(\vartheta \mathcal{X}\left(u_{i}\right), \zeta \mathcal{X}\left(u_{i}\right)\right): i=1,2, \ldots, m\right\}$. Define the first type of PF lower approximation (1-PFLA) $\mathcal{L}_{1}^{P F}(\mathcal{X})$ and the first type of PF upper approximation (1-PFUA) $\mathcal{U}_{1}^{P F}(\mathcal{X})$ as follows.

$\mathcal{L}_{1}^{P F}(\mathcal{X})=\left\{\begin{array}{r}\left(u_{i}, \wedge_{t=1}^{n}\left(\zeta_{\widetilde{\mathcal{P}}_{u}^{\beta}}\left(u_{i}, u_{j}\right) \vee \vartheta \mathcal{X}\left(u_{j}\right)\right),\right. \\ \left.\vee_{i=1}^{n}\left(\vartheta_{\widetilde{\mathcal{P}}_{u}^{\beta}}\left(u_{i}, u_{j}\right) \wedge \zeta \mathcal{X}\left(u_{j}\right)\right)\right)\end{array}\right\}$,
$\mathcal{U}_{1}^{P F}(\mathcal{X})=\left\{\begin{array}{r}\left(u_{i}, \vee_{i=1}^{n}\left(\vartheta_{\widetilde{\mathcal{P}}_{u}^{\beta}}\left(u_{i}, u_{j}\right) \wedge \vartheta \mathcal{X}\left(u_{j}\right)\right),\right. \\ \left.\wedge_{i=1}^{n}\left(\zeta_{\widetilde{\mathcal{P}}_{u}^{\beta}}\left(u_{i}, u_{j}\right) \vee \zeta \mathcal{X}\left(u_{j}\right)\right)\right)\end{array}\right\}$

Then the pair $\left(\mathcal{L}_{1}^{P F}(\mathcal{X}), \mathcal{U}_{1}^{P F}(\mathcal{X})\right)$ is called the $1-\mathrm{PF} \beta \mathrm{CRS}$.

Definition 8 Consider $\Theta$ be a universe. For every $u \in \Theta$, if we have a membership function $\mu_{\mathcal{X}}: \Theta \rightarrow[0,1]$ and a non-membership function $v_{\mathcal{X}}: \Theta \rightarrow[0,1]$. Define the q-ROFS $\mathcal{X}$ as indicated below.

$\mathcal{X}=\left\{\left(u, \mu_{\mathcal{X}}(u), v_{\mathcal{X}}(u)\right)\right\}$,

where $0 \leq \mu_{\mathcal{X}}^{q}(u)+v_{\mathcal{X}}^{q}(u) \leq 1$

Definition 9 [53] Consider a q-ROFS $\mathcal{X} \in \Theta$, define the grade of indeterminacy of $u \in \Theta$ to $\mathcal{X}$ as follows.

$\Xi_{\mathcal{X}}(u)=\sqrt[q]{1-\vartheta_{\mathcal{P}}^{q}(u)-\zeta_{\mathcal{P}}^{q}(u)}$

Definition 10 [53] Let $\mathcal{X}_{1}=\left(\mu_{\mathcal{X}_{1}}, v_{\mathcal{X}_{1}}\right)$ and $\mathcal{X}_{2}=\left(\mu_{\mathcal{X}_{2}}\right.$, $v_{\mathcal{X}_{2}}$ ), for $\mathcal{X}_{1}, \mathcal{X}_{2} \in \mathrm{q}-\operatorname{ROF}(\Theta)$. Then $\forall u \in \Theta$, we have the following properties

(1) $\mathcal{X}_{1}^{c}=\left\{\left(u, v_{\mathcal{X}_{1}}(u), \mu_{\mathcal{X}_{1}}(u)\right)\right\}$.

(2) $\mathcal{X}_{1}=\mathcal{X}_{2} \Longleftrightarrow \mu_{\mathcal{X}_{1}}(u)=\mu_{\mathcal{X}_{2}}(u)$ and $v_{\mathcal{X}_{1}}(u)=$ $v_{\mathcal{X}_{2}}(u)$.

(3) $\mathcal{X}_{1} \subseteq \mathcal{X}_{2}$ if $\mu \mathcal{X}_{1}(u) \leq \mu \mathcal{X}_{2}(u)$ and $\nu_{\mathcal{X}_{1}}(u) \leq v_{\mathcal{X}_{2}}(u)$.

(4) $\mathcal{X}_{1} \cap \mathcal{X}_{2}=\left\{\left(u, \mu \mathcal{X}_{1}(u) \wedge \mu \mathcal{X}_{2}(u), v_{\mathcal{X}_{1}}(u) \vee v_{\mathcal{X}_{2}}(u)\right)\right\}$.

(5) $\mathcal{X}_{1} \cup \mathcal{X}_{2}=\left\{\left(u, \mu_{\mathcal{X}_{1}}(u) \vee \mu \mathcal{X}_{2}(u), v_{\mathcal{X}_{1}}(u) \wedge v_{\mathcal{X}_{2}}(u)\right)\right\}$. 
(6) $\mathcal{X}_{1}-\mathcal{X}_{2}=\mathcal{X}_{1} \cap \mathcal{X}_{2}^{c}$.

Hussian et al. [57] presented the concept of CPFRS with the aid of the definition of $\mathrm{q}-\mathrm{ROF} \beta$-neighborhood as indicated below.

Definition 11 [57] Consider $\Theta$ be a universe and $\widetilde{\Re}=$ $\left\{\widetilde{\mathcal{R}}_{1}, \widetilde{\mathcal{R}}_{2}, \ldots, \widetilde{\mathcal{R}}_{m}\right\}$ where $\widetilde{\mathcal{R}}_{i} \in \mathrm{q}-\operatorname{ROF}(\Theta)$ and $i=$ $1,2, \ldots, m$. Then for each q-ROFN $\beta=\left(\mu_{\beta}, v_{\beta}\right)$, a q-ROF $\beta$ covering of $\Theta$, if $\widetilde{\mathcal{R}}_{i}(u) \geq \beta$ for each $u \in \Theta$. Now $(\Theta, \widetilde{\mathcal{R}})$ is called the $\mathrm{q}-\mathrm{ROF} \beta$-covering approximation space (briefly, $\mathrm{q}-\mathrm{ROF} \beta \mathrm{CAS})$.

Definition 12 [57] Consider a q-ROF $\beta \operatorname{CAS}(\Theta, \widetilde{\Re})$, for some q-ROFN $\beta=\left(\mu_{\beta}, v_{\beta}\right)$. For every $u \in \Theta$, define the q-ROF $\beta$-neighborhood of $u$ as follows.

$\widetilde{\mathcal{Q}}_{u}^{\beta}=\cap\left\{\widetilde{\mathcal{R}}_{i} \in \widetilde{\mathfrak{R}}: \widetilde{\mathcal{R}}_{i}(u) \geq \beta\right\}$.

Definition 13 [57] Consider a q-ROF $\beta \operatorname{CAS}(\Theta, \widetilde{\Re})$, for some q-ROFN $\beta=\left(\mu_{\beta}, v_{\beta}\right)$. For every $u \in \Theta$ and $\mathcal{X} \in \mathrm{q}-\operatorname{ROF}(\Lambda)$, that is, $\mathcal{X}=\left\{\left(\mu_{\mathcal{X}}\left(u_{i}\right), \nu_{\mathcal{X}}\left(u_{i}\right)\right): i=\right.$ $1,2, \ldots, m\}$. Define the first type of q-ROF lower approximation (1-q-ROFLA) $\mathcal{L}_{1}^{\mathrm{q}-\mathrm{ROF}}(\mathcal{X})$ and the first type of q-ROF upper approximation (1-q-ROFUA) $\mathcal{U}_{1}^{\mathrm{q}-\mathrm{ROF}}(\mathcal{X})$ as follows.

$$
\begin{array}{r}
\mathcal{L}_{1}^{\mathrm{q}-\mathrm{ROF}}(\mathcal{X})=\left\{\begin{array}{r}
\left(u_{k}, \wedge_{k=1}^{n}\left(\mu_{\widetilde{\mathcal{Q}}_{u_{k}}^{\beta}}\left(u_{k}, u_{r}\right) \wedge \mu \mathcal{X}\left(u_{r}\right)\right),\right. \\
\left.\vee_{k=1}^{n}\left(v_{\mathcal{Q}_{u_{k}}^{\beta}}\left(u_{k}, u_{r}\right) \vee v \mathcal{X}\left(u_{r}\right)\right)\right)_{q}
\end{array}\right\}, \\
\mathcal{U}_{1}^{\mathrm{q}-\mathrm{ROF}}(\mathcal{X})=\left\{\begin{array}{c}
\left(u_{k}, \vee_{k=1}^{n}\left(\mu_{\widetilde{\mathcal{Q}}_{u_{k}}^{\beta}}\left(u_{k}, u_{r}\right) \vee \mu \mathcal{X}\left(u_{r}\right)\right),\right. \\
\left.\wedge_{k=1}^{n}\left(v_{\mathcal{Q}_{u_{k}}^{\beta}}\left(u_{k}, u_{r}\right) \wedge v \mathcal{X}\left(u_{r}\right)\right)\right)_{q}
\end{array}\right\}
\end{array}
$$

Then the pair $\left(\mathcal{L}_{1}^{\mathrm{q}-\mathrm{ROF}}(\mathcal{X}), \mathcal{U}_{1}^{\mathrm{q}-\mathrm{ROF}}(\mathcal{X})\right)$ is called the 1 -qROF $\beta$ CRS.

Definition 14 [57] Let $\mathcal{X}_{1}=\left(\mu \mathcal{X}_{1}, \nu_{\mathcal{X}_{1}}\right)$ and $\mathcal{X}_{2}=\left(\mu \mathcal{X}_{2}\right.$, $\left.v_{\mathcal{X}_{2}}\right)$ be two q-ROFNs. Then the distances among them is defined as follows $\forall q \geq 1$.

$$
\begin{aligned}
\mathcal{E}\left(\mathcal{X}_{1}, \mathcal{X}_{2}\right)= & {\left[\frac{1}{2 n} \sum_{u \in \Theta}\left|\mu \mathcal{X}_{1}(u)-\mu \mathcal{X}_{2}(u)\right|^{q}\right.} \\
& \left.+\frac{1}{2 n} \sum_{u \in \Theta}\left|\nu_{\mathcal{X}_{1}}(u)-v_{\mathcal{X}_{2}}(u)\right|^{q}\right]^{\frac{1}{q}} .
\end{aligned}
$$

\section{PF complementary $\beta$-neighborhood and three novel kinds of CPFRS}

This section's objective is to investigate the definition of PF complementary $\beta$-neighborhood and then construct three new types of a CPFRS model. Further, we discuss the relationships via these models.
Table 1 Rating for $(\Lambda, \tilde{\Upsilon})$

\begin{tabular}{llllll}
\hline & $\widetilde{\Delta}_{1}$ & $\widetilde{\Delta}_{2}$ & $\widetilde{\Delta}_{3}$ & $\widetilde{\Delta}_{4}$ & $\widetilde{\Delta}_{5}$ \\
\hline$u_{1}$ & $(0.8,0.2)$ & $(0.7,0.6)$ & $(0.5,0.8)$ & $(0.9,0.3)$ & $(0.4,0.6)$ \\
$u_{2}$ & $(0.7,0.3)$ & $(0.4,0.5)$ & $(0.8,0.1)$ & $(0.6,0.7)$ & $(0.7,0.2)$ \\
$u_{3}$ & $(0.6,0.4)$ & $(0.8,0.6)$ & $(0.8,0.3)$ & $(0.3,0.9)$ & $(0.7,0.1)$ \\
$u_{4}$ & $(0.8,0.3)$ & $(0.3,0.9)$ & $(0.4,0.3)$ & $(0.7,0.6)$ & $(0.8,0.4)$ \\
$u_{5}$ & $(0.2,0.7)$ & $(0.7,0.3)$ & $(0.9,0.2)$ & $(0.7,0.5)$ & $(0.8,0.3)$ \\
$u_{6}$ & $(0.6,0.6)$ & $(0.7,0.2)$ & $(0.6,0.5)$ & $(0.8,0.1)$ & $(0.4,0.2)$ \\
\hline
\end{tabular}

\section{PF complementary $\beta$-neighborhood}

Definition 15 Consider a $\operatorname{PF} \beta \operatorname{CAS}(\Lambda, \widetilde{\Upsilon})$ and PFN $\beta=$ $\left(\vartheta_{\beta}, \zeta_{\beta}\right)$. Then for each $u \in \Lambda$, define the PF complementary $\beta$-neighborhood of $u$ as follows.

$\hat{\widetilde{\mathcal{P}}}_{u}^{\beta}(v)=\widetilde{\mathcal{P}}_{v}^{\beta}(u), \quad \forall v \in \Lambda$.

Example 1 ConsideraPF $\beta \operatorname{CAS}(\Lambda, \tilde{\Upsilon}), \Lambda=\left\{u_{1}, u_{2}, u_{3}, u_{4}\right.$, $\left.u_{5}, u_{6}\right\}$ and $\widetilde{\Upsilon}=\left\{\widetilde{\Delta}_{1}, \widetilde{\Delta}_{2}, \widetilde{\Delta}_{3}, \widetilde{\Delta}_{4}, \widetilde{\Delta}_{5}\right\}$, where $\beta=$ $(0.7,0.4)$ are summarized in Table 1.

It is computed that

$\widetilde{\mathcal{P}}_{u_{1}}^{(0.7,0.4)}=\widetilde{\Delta}_{1} \cap \widetilde{\Delta}_{4}$

$\widetilde{\mathcal{P}}_{u_{2}}^{(0.7,0.4)}=\widetilde{\Delta}_{1} \cap \widetilde{\Delta}_{3} \cap \widetilde{\Delta}_{5}$,

$\widetilde{\mathcal{P}}_{u_{3}}^{(0.7,0.4)}=\widetilde{\Delta}_{3} \cap \widetilde{\Delta}_{5}$,

$\widetilde{\mathcal{P}}_{u_{4}}^{(0.7,0.4)}=\widetilde{\Delta}_{1} \cap \widetilde{\Delta}_{5}$,

$\widetilde{\mathcal{P}}_{u_{5}}^{(0.7,0.4)}=\widetilde{\Delta}_{2} \cap \widetilde{\Delta}_{3} \cap \widetilde{\Delta}_{5}$,

$\widetilde{\mathcal{P}}_{u_{6}}^{(0.7,0.4)}=\widetilde{\Delta}_{2} \cap \widetilde{\Delta}_{4}$

The rating corresponding to them are computed and listed in Table 2. However, the values of their complement $\widetilde{\mathcal{P}}_{u}^{(0 . \hat{7}, 0.4)}$ is given in Table 3.

\section{The first type of a CPFRS}

Definition 16 Consider a $\operatorname{PF} \beta \operatorname{CAS}(\Lambda, \tilde{\Upsilon})$ and PFN $\beta=$ $\left(\vartheta_{\beta}, \zeta_{\beta}\right)$. For all $u \in \Lambda$ and $\mathcal{X} \in \operatorname{PF}(\Lambda)$. Define the 2-PFLA $\mathcal{L}_{2}^{\mathrm{PF}}(\mathcal{X})$ and 2-PFUA $\mathcal{U}_{2}^{\mathrm{PF}}(\mathcal{X})$ as follows.

$$
\begin{aligned}
& \mathcal{L}_{2}^{\mathrm{PF}}(\mathcal{X})=\left\{\begin{array}{r}
\left(u_{i}, \wedge_{i=1}^{n}\left(\zeta_{\hat{\mathcal{P}}_{u}^{\beta}}\left(u_{i}, u_{j}\right) \vee \vartheta \mathcal{X}\left(u_{j}\right)\right),\right. \\
\left.\vee_{i=1}^{n}\left(\vartheta_{\hat{\mathcal{P}}_{u}^{\beta}}\left(u_{i}, u_{j}\right) \wedge \zeta_{\mathcal{X}}\left(u_{j}\right)\right)\right)
\end{array}\right\},
\end{aligned}
$$

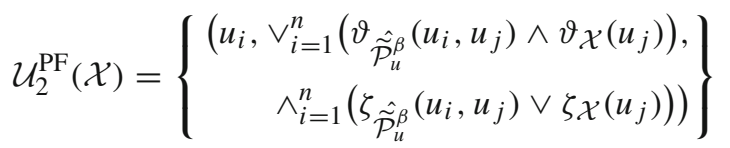

Then the pair $\left(\mathcal{L}_{2}^{\mathrm{PF}}(\mathcal{X}), \mathcal{U}_{2}^{\mathrm{PF}}(\mathcal{X})\right)$ is called the 2-PF $\beta$ CRS. 
Table 2 Result for

$\widetilde{\mathcal{P}}_{u_{s}}^{(0.7,0.4)}, s=1,2, \ldots, 6$

\begin{tabular}{lllllll}
\hline & $u_{1}$ & $u_{2}$ & $u_{3}$ & $u_{4}$ & $u_{5}$ & $u_{6}$ \\
\hline$\widetilde{\mathcal{P}}_{u_{1}}^{\beta}$ & $(0.8,0.3)$ & $(0.6,0.7)$ & $(0.3,0.9)$ & $(0.7,0.6)$ & $(0.2,0.7)$ & $(0.6,0.6)$ \\
$\widetilde{\mathcal{P}}_{u_{2}}^{\beta}$ & $(0.4,0.8)$ & $(0.7,0.3)$ & $(0.6,0.4)$ & $(0.4,0.4)$ & $(0.2,0.7)$ & $(0.4,0.6)$ \\
$\widetilde{\mathcal{P}}_{u_{3}}^{\beta}$ & $(0.4,0.8)$ & $(0.7,0.2)$ & $(0.7,0.3)$ & $(0.4,0.4)$ & $(0.8,0.3)$ & $(0.4,0.5)$ \\
$\widetilde{\mathcal{P}}_{u_{4}}^{\beta}$ & $(0.4,0.6)$ & $(0.7,0.3)$ & $(0.6,0.4)$ & $(0.8,0.4)$ & $(0.2,0.7)$ & $(0.4,0.6)$ \\
$\widetilde{\mathcal{P}}_{u_{5}}^{\beta}$ & $(0.4,0.8)$ & $(0.4,0.5)$ & $(0.7,0.6)$ & $(0.3,0.9)$ & $(0.7,0.3)$ & $(0.4,0.5)$ \\
$\widetilde{\mathcal{P}}_{u_{6}}^{\beta}$ & $(0.7,0.6)$ & $(0.4,0.7)$ & $(0.3,0.9)$ & $(0.3,0.9)$ & $(0.7,0.5)$ & $(0.7,0.2)$ \\
\hline
\end{tabular}

Table 3 Result for $\widetilde{\mathcal{P}}_{u_{s}}^{(0.7,0.4)}, s=1,2, \ldots, 6$

\begin{tabular}{lllllll}
\hline & $u_{1}$ & $u_{2}$ & $u_{3}$ & $u_{4}$ & $u_{5}$ & $u_{6}$ \\
\hline$\hat{\widetilde{P}}_{u_{1}}^{\beta}$ & $(0.3,0.8)$ & $(0.7,0.6)$ & $(0.9,0.3)$ & $(0.6,0.7)$ & $(0.7,0.2)$ & $(0.6,0.6)$ \\
$\hat{\widetilde{\mathcal{P}}_{u_{2}}^{\beta}}$ & $(0.8,0.4)$ & $(0.3,0.7)$ & $(0.4,0.6)$ & $(0.4,0.4)$ & $(0.7,0.2)$ & $(0.6,0.4)$ \\
$\hat{\widetilde{\mathcal{P}}_{u_{3}}^{\beta}}$ & $(0.8,0.4)$ & $(0.2,0.7)$ & $(0.3,0.7)$ & $(0.4,0.4)$ & $(0.3,0.8)$ & $(0.5,0.4)$ \\
$\hat{\widetilde{\mathcal{P}}}_{u_{4}}^{\beta}$ & $(0.6,0.4)$ & $(0.3,0.7)$ & $(0.4,0.6)$ & $(0.4,0.8)$ & $(0.7,0.2)$ & $(0.6,0.4)$ \\
$\hat{\widetilde{\mathcal{P}}}_{u_{5}}^{\beta}$ & $(0.8,0.4)$ & $(0.5,0.4)$ & $(0.6,0.7)$ & $(0.9,0.3)$ & $(0.3,0.7)$ & $(0.5,0.4)$ \\
$\tilde{\widetilde{\mathcal{P}}}_{u_{6}}^{\beta}$ & $(0.6,0.7)$ & $(0.7,0.4)$ & $(0.9,0.3)$ & $(0.9,0.3)$ & $(0.5,0.7)$ & $(0.2,0.7)$ \\
\hline
\end{tabular}

Example 2 Consider Example 1 and $\mathcal{X}=\left(u_{1}, 0.7,0.4\right)+$ $\left(u_{2}, 0.4,0.7\right)+\left(u_{3}, 0.8,0.6\right)+\left(u_{4}, 0.8,0.1\right)+\left(u_{5}, 0.6,0.5\right)$ $+\left(u_{6}, 0.9,0.1\right)$. Then the following results hold.

(1) $\mathcal{L}_{1}^{\mathrm{PF}}(\mathcal{X})=\left(u_{1}, 0.7,0.6\right)+\left(u_{2}, 0.4,0.7\right)+\left(u_{3}, 0.4,0.7\right)$ $+\left(u_{4}, 0.4,0.7\right)+\left(u_{5}, 0.5,0.6\right)+\left(u_{6}, 0.6,0.5\right)$, $\mathcal{U}_{1}^{\mathrm{PF}}(\mathcal{X})=\left(u_{1}, 0.7,0.4\right)+\left(u_{2}, 0.6,0.4\right)+\left(u_{3}, 0.7,0.4\right)$ $+\left(u_{4}, 0.8,0.4\right)+\left(u_{5}, 0.7,0.5\right)+\left(u_{6}, 0.7,0.2\right)$.

(2) $\mathcal{L}_{2}^{\mathrm{PF}}(\mathcal{X})=\left(u_{1}, 0.6,0.7\right)+\left(u_{2}, 0.6,0.5\right)+\left(u_{3}, 0.7,0.4\right)$ $+\left(u_{4}, 0.6,0.5\right)+\left(u_{5}, 0.4,0.6\right)+\left(u_{6}, 0.4,0.7\right)$, $\mathcal{U}_{2}^{\mathrm{PF}}(\mathcal{X})=\left(u_{1}, 0.8,0.5\right)+\left(u_{2}, 0.7,0.4\right)+\left(u_{3}, 0.7,0.4\right)$ $+\left(u_{4}, 0.6,0.4\right)+\left(u_{5}, 0.8,0.3\right)+\left(u_{6}, 0.8,0.3\right)$.

Theorem 1 Consider a PF $\beta C A S(\Lambda, \widetilde{\Upsilon})$. Then, we have the following properties

(1) $\mathcal{L}_{2}^{P F}(\mathcal{X})=\left(\mathcal{U}_{2}^{P F}\left(\mathcal{X}^{c}\right)\right)^{c}$.

(2) $\mathcal{U}_{2}^{P F}(\mathcal{X})=\left(\mathcal{L}_{2}^{P F}\left(\mathcal{X}^{c}\right)\right)^{c}$.

(3) If $\mathcal{X} \subseteq \mathcal{Y}$, then $\mathcal{L}_{2}^{P F}(\mathcal{X}) \subseteq \mathcal{L}_{2}^{P F}(\mathcal{Y})$.

(4) If $\mathcal{X} \subseteq \mathcal{Y}$, then $\mathcal{U}_{2}^{P F}(\mathcal{X}) \subseteq \mathcal{U}_{2}^{P F}(\mathcal{Y})$.

(5) $\mathcal{L}_{2}^{P F}(\mathcal{X} \cap \mathcal{Y})=\mathcal{L}_{2}^{P F}(\mathcal{X}) \cap \mathcal{L}_{2}^{P F}(\mathcal{Y})$.

(6) $\mathcal{U}_{2}^{P F}(\mathcal{X} \cap \mathcal{Y}) \subseteq \mathcal{U}_{2}^{P F}(\mathcal{X}) \cap \mathcal{U}_{2}^{P F}(\mathcal{Y})$.

(7) $\mathcal{L}_{2}^{P F}(\mathcal{X} \cup \mathcal{Y}) \supseteq \mathcal{L}_{2}^{P F}(\mathcal{X}) \cup \mathcal{L}_{2}^{P F}(\mathcal{Y})$.

(8) $\mathcal{U}_{2}^{P F}(\mathcal{X} \cup \mathcal{Y})=\mathcal{U}_{2}^{P F}(\mathcal{X}) \cup \mathcal{U}_{2}^{P F}(\mathcal{Y})$.

Proof of Theorem 1 We just prove (1), (3), (5) and (7).
(1) $\mathcal{L}_{2}^{P F}\left(\mathcal{X}^{c}\right)$

$$
\begin{aligned}
& =\left\{\begin{array}{r}
\left(u_{s}, \wedge_{s=1}^{n}\left(\zeta_{\hat{\mathcal{P}}_{u}^{\beta}}\left(u_{s}, u_{t}\right) \vee \vartheta \mathcal{X}^{c}\left(u_{t}\right)\right),\right. \\
\left.\vee_{s=1}^{n}\left(\vartheta_{\hat{\mathcal{P}}_{u}^{\beta}}\left(u_{s}, u_{t}\right) \wedge \zeta \mathcal{X}^{c}\left(u_{t}\right)\right)\right)
\end{array}\right\} \\
& =\left\{\begin{array}{r}
\left(u_{s}, \wedge_{s=1}^{n}\left(\zeta_{\hat{\mathcal{P}}_{u}^{\beta}}\left(u_{s}, u_{t}\right) \vee\left(1-\vartheta \mathcal{X}\left(u_{t}\right)\right)\right),\right. \\
\left.\vee_{s=1}^{n}\left(\vartheta_{\hat{\mathcal{P}}_{u}^{\beta}}\left(u_{s}, u_{t}\right) \wedge\left(1-\zeta \mathcal{X}\left(u_{t}\right)\right)\right)\right)
\end{array}\right\} \\
& =\left(\mathcal{U}_{2}^{P F}(\mathcal{X})\right)^{c}
\end{aligned}
$$

(3) Let $\mathcal{X}, \mathcal{Y} \in \operatorname{PF}(\Lambda)$ such that $\mathcal{X} \subseteq \mathcal{Y}$ (i.e., $\vartheta \mathcal{X} \leq \vartheta \mathcal{Y}$ and $\zeta \mathcal{X} \geq \zeta \mathcal{Y})$ and $u \in \Lambda$. Then we get the following result.

$$
\mathcal{L}_{2}^{P F}(\mathcal{X})(u)=\left\{\begin{array}{r}
\left(u_{s}, \wedge_{s=1}^{n}\left(\zeta_{\hat{\mathcal{P}}_{u}^{\beta}}\left(u_{s}, u_{t}\right) \vee \vartheta_{\mathcal{X}}\left(u_{t}\right)\right),\right. \\
\left.\vee_{s=1}^{n}\left(\vartheta_{\hat{\mathcal{P}}_{u}^{\beta}}\left(u_{s}, u_{t}\right) \wedge \zeta_{\mathcal{X}}\left(u_{t}\right)\right)\right)
\end{array}\right\}
$$

Now for $\mathcal{X} \subseteq \mathcal{Y}$, we have

$$
\begin{aligned}
\vartheta_{\mathcal{L}_{2}^{P F}(\mathcal{X})} & =\wedge_{s=1}^{n}\left(\vartheta_{\hat{\mathcal{P}}_{u}^{\beta}}\left(u_{s}, u_{t}\right) \vee \vartheta \mathcal{X}\left(u_{t}\right)\right) \\
& \leq \wedge_{s=1}^{n}\left(\vartheta_{\tilde{\mathcal{P}}_{u}^{\beta}}\left(u_{s}, u_{t}\right) \vee \vartheta_{\mathcal{Y}}\left(u_{t}\right)\right) \\
& =\vartheta_{\mathcal{L}_{2}^{P F}(\mathcal{Y})}
\end{aligned}
$$

and

$$
\begin{aligned}
\zeta_{\mathcal{L}_{2}^{P F}(\mathcal{X})} & =\vee_{s=1}^{n}\left(\vartheta_{\hat{\mathcal{P}}_{u}^{\beta}}\left(u_{s}, u_{t}\right) \wedge \zeta \mathcal{X}\left(u_{t}\right)\right) \\
& \geq \vee_{s=1}^{n}\left(\vartheta_{\hat{\mathcal{P}}_{u}^{\beta}}\left(u_{s}, u_{t}\right) \wedge \zeta \mathcal{Y}\left(u_{t}\right)\right) \\
& =\zeta_{\mathcal{L}_{2}^{P F}(\mathcal{Y})}
\end{aligned}
$$


Therefore, $\mathcal{L}_{2}^{P F}(\mathcal{X}) \subseteq \mathcal{L}_{2}^{P F}(\mathcal{Y})$.

(5)

$$
\begin{aligned}
& \mathcal{L}_{2}^{P F}(\mathcal{X} \cap \mathcal{Y}) \\
& =\left\{\begin{array}{r}
\left(u_{s}, \wedge_{s=1}^{n}\left(\zeta_{\hat{\mathcal{P}}_{u}^{\beta}}\left(u_{s}, u_{t}\right) \vee \vartheta \mathcal{X} \cap \mathcal{Y}\left(u_{t}\right)\right),\right. \\
\left.\vee_{s=1}^{n}\left(\vartheta_{\hat{\mathcal{P}}_{u}^{\beta}}\left(u_{s}, u_{t}\right) \wedge \zeta \mathcal{X} \cap \mathcal{Y}\left(u_{t}\right)\right)\right)
\end{array}\right\} \\
& =\left\{\begin{array}{r}
\left(u_{s}, \wedge_{s=1}^{n}\left(\zeta_{\tilde{\mathcal{P}}_{u_{s}}^{\beta}}\left(u_{s}, u_{t}\right) \vee\left(\vartheta \mathcal{X}\left(u_{t}\right) \cap \vartheta \mathcal{Y}\left(u_{t}\right)\right)\right),\right. \\
\left.\vee_{t=1}^{m}\left(\vartheta_{\tilde{\mathcal{P}}_{u_{s}}^{\beta}}\left(u_{s}, u_{t}\right) \wedge\left(\zeta \mathcal{X}\left(u_{t}\right) \cap \zeta \mathcal{Y}\left(u_{t}\right)\right)\right)\right)
\end{array}\right\} \\
& =\left\{\begin{array}{r}
\left(u_{s}, \wedge_{s=1}^{n}\left(\zeta_{\tilde{\mathcal{P}}_{u_{s}^{\beta}}^{\beta}}\left(u_{s}, u_{t}\right) \vee \vartheta \mathcal{X}\left(u_{t}\right)\right)\right. \\
\wedge \wedge_{s=1}^{n}\left(\zeta_{\tilde{\mathcal{P}}_{u_{s}}^{\beta}}\left(u_{s}, u_{t}\right) \vee \vartheta \mathcal{Y}\left(u_{t}\right)\right), \\
\vee_{t=1}^{m}\left(\vartheta_{\hat{\mathcal{P}}_{u_{s}}^{\beta}}\left(u_{s}, u_{t}\right) \wedge \zeta \mathcal{X}\left(u_{t}\right)\right) \\
\left.\wedge \vee_{t=1}^{m}\left(\vartheta_{\tilde{\mathcal{P}}_{u_{s}}^{\beta}}\left(u_{s}, u_{t}\right) \wedge \zeta \mathcal{Y}\left(u_{t}\right)\right)\right)
\end{array}\right\} \\
& =\mathcal{L}_{2}^{P F}(\mathcal{X}) \cap \mathcal{L}_{2}^{P F}(\mathcal{Y})
\end{aligned}
$$

$$
\begin{aligned}
& \mathcal{L}_{2}^{P F}(\mathcal{X} \cup \mathcal{Y}) \\
& =\left\{\begin{array}{r}
\left(u_{s}, \wedge_{s=1}^{n}\left(\zeta_{\hat{\mathcal{P}}_{u_{s}}^{\beta}}\left(u_{s}, u_{t}\right) \vee \vartheta_{(\mathcal{X} \cup \mathcal{Y})}\left(u_{t}\right)\right),\right. \\
\left.\vee_{s=1}^{n}\left(\vartheta_{\hat{\mathcal{P}}_{u_{s}}^{\beta}}\left(u_{s}, u_{t}\right) \wedge \zeta_{(\mathcal{X} \cup \mathcal{Y})}\left(u_{t}\right)\right)\right)
\end{array}\right\} \\
& =\left\{\begin{array}{r}
\left(u_{s}, \wedge_{s=1}^{n}\left(\zeta_{\tilde{\mathcal{P}}_{u_{s}}^{\beta}}\left(u_{s}, u_{t}\right)\right.\right. \\
\left.\vee\left(\vartheta \mathcal{X}\left(u_{t}\right) \cup \vartheta_{\mathcal{Y}}\left(u_{t}\right)\right)\right), \\
\vee_{s=1}^{m}\left(\vartheta_{\tilde{\mathcal{P}}_{u_{s}}^{\beta}}\left(u_{s}, u_{t}\right)\right. \\
\left.\left.\wedge\left(\zeta \mathcal{X}\left(u_{t}\right) \cup \zeta_{\mathcal{Y}}\left(u_{t}\right)\right)\right)\right)
\end{array}\right\} \\
& \geq\left\{\begin{array}{r}
\left(u_{s}, \wedge_{s=1}^{n}\left(\zeta_{\tilde{\mathcal{P}}_{u_{s}}^{\beta}}\left(u_{s}, u_{t}\right) \vee \vartheta \mathcal{X}\left(u_{t}\right)\right)\right. \\
\vee \wedge_{s=1}^{n}\left(\zeta_{\tilde{\mathcal{P}}_{u_{s}}^{\beta}}\left(u_{s}, u_{t}\right) \vee \vartheta \mathcal{Y}\left(u_{t}\right)\right), \\
\vee_{t=1}^{m}\left(\vartheta_{\tilde{\mathcal{P}}_{u_{s}}^{\beta}}\left(u_{s}, u_{t}\right) \wedge \zeta \mathcal{X}\left(u_{t}\right)\right) \\
\left.\wedge \vee_{t=1}^{m}\left(\vartheta_{\hat{\mathcal{P}_{u s}^{\beta}}}^{\hat{u}_{u_{s}}}\left(u_{s}, u_{t}\right) \wedge \zeta \mathcal{Y}\left(u_{t}\right)\right)\right)
\end{array}\right\} \\
& =\mathcal{L}_{2}^{P F}(\mathcal{X}) \cup \mathcal{L}_{2}^{P F}(\mathcal{Y})
\end{aligned}
$$

Definition 17 Consider a $\operatorname{PF} \beta \operatorname{CAS}(\Lambda, \tilde{\Upsilon})$ and $\operatorname{PFN} \beta=$ $\left(\vartheta_{\beta}, \zeta_{\beta}\right)$. Thus the rough and precision degrees of $\mathcal{X} \in$ $\operatorname{PF}(\Lambda)$ are respectively seen as follows.

$$
\begin{aligned}
{ }_{2} \Re_{\tilde{\mathcal{P}}^{\beta}(\mathcal{X})} & =\mathcal{D}\left(\mathcal{L}_{2}^{\mathrm{PF}}(\mathcal{X}), \mathcal{U}_{2}^{\mathrm{PF}}(\mathcal{X})\right), \\
{ }_{2} \mathscr{P}_{\tilde{\mathcal{P}}^{\beta}(\mathcal{X})} & =1-{ }_{2} \Re_{\hat{\mathcal{P}} \beta}(\mathcal{X})
\end{aligned}
$$

Example 3 Consider Examples 1 and 2. Then the following results hold.

${ }_{2} \Re_{\tilde{\mathcal{P}}^{\beta}(\mathcal{X})}=0.248, \quad 2 \mathscr{P}_{\hat{\mathcal{P}}^{\beta}(\mathcal{X})}=0.752$

\section{The second type of a CPFRS}

Definition 18 Consider a $\operatorname{PF} \beta \operatorname{CAS}(\Lambda, \tilde{\Upsilon})$ and $\operatorname{PFN} \beta=$ $\left(\vartheta_{\beta}, \zeta_{\beta}\right)$. Then for each $u \in \Lambda$, define a new kind of PF $\beta$-neighborhood as follows.

${ }_{1} \widetilde{\mathbb{I}}_{u}^{\beta}=\widetilde{\mathcal{P}}_{u}^{\beta} \wedge \hat{\widetilde{\mathcal{P}}}_{u}^{\beta}$

Example 4 Consider Example 1. We compute ${ }_{1} \widetilde{\mathbb{d}}_{u}^{\beta}$ as set in Table 4.

Definition 19 Consider a $\operatorname{PF} \beta \operatorname{CAS}(\Lambda, \tilde{\Upsilon})$ and $\operatorname{PFN} \beta=$ $\left(\vartheta_{\beta}, \zeta_{\beta}\right)$. For all $u \in \Lambda$ and $\mathcal{X} \in \operatorname{PF}(\Lambda)$. Define the 3-PFLA $\mathcal{L}_{3}^{\mathrm{PF}}(\mathcal{X})$ and 3-PFUA $\mathcal{U}_{3}^{\mathrm{PF}}(\mathcal{X})$ as follows.

$$
\begin{aligned}
& \mathcal{L}_{3}^{\mathrm{PF}}(\mathcal{X})=\left\{\begin{array}{r}
\left(u_{i}, \wedge_{i=1}^{n}\left(\zeta_{1} \widetilde{\mathbb{\Psi}}_{u}^{\beta}\left(u_{i}, u_{j}\right) \vee \vartheta_{\mathcal{X}}\left(u_{j}\right)\right),\right. \\
\left.\vee_{i=1}^{n}\left(\vartheta_{1{ }_{1}^{\beta}}^{\beta}\left(u_{i}, u_{j}\right) \wedge \zeta_{\mathcal{X}}\left(u_{j}\right)\right)\right)
\end{array}\right\}, \\
& \mathcal{U}_{3}^{\mathrm{PF}}(\mathcal{X})=\left\{\begin{array}{r}
\left(u_{i}, \vee_{i=1}^{n}\left(\vartheta_{1 \widetilde{\mathbb{T}}_{u}^{\beta}}\left(u_{i}, u_{j}\right) \wedge \vartheta_{\mathcal{X}}\left(u_{j}\right)\right),\right. \\
\left.\wedge_{i=1}^{n}\left(\zeta_{1} \widetilde{\mathbb{\Psi}}_{u}^{\beta}\left(u_{i}, u_{j}\right) \vee \zeta \mathcal{X}\left(u_{j}\right)\right)\right)
\end{array}\right\} .
\end{aligned}
$$

Then the pair $\left(\mathcal{L}_{3}^{\mathrm{PF}}(\mathcal{X}), \mathcal{U}_{3}^{\mathrm{PF}}(\mathcal{X})\right)$ is called the 3-PF $\beta$ CRS.

Example 5 Consider Examples 1 and 2. Then we have the following outcomes.

$$
\begin{gathered}
\mathcal{L}_{3}^{\mathrm{PF}}(\mathcal{X})=\left(u_{1}, 0.7,0.6\right)+\left(u_{2}, 0.7,0.4\right) \\
+\left(u_{3}, 0.7,0.4\right)+\left(u_{4}, 0.7,0.4\right) \\
+\left(u_{5}, 0.5,0.6\right)+\left(u_{6}, 0.7,0.5\right) \\
\mathcal{U}_{3}^{\mathrm{PF}}(\mathcal{X})=\left(u_{1}, 0.6,0.6\right)+\left(u_{2}, 0.4,0.4\right) \\
+\left(u_{3}, 0.4,0.4\right)+\left(u_{4}, 0.4,0.6\right) \\
+\left(u_{5}, 0.6,0.5\right)+\left(u_{6}, 0.6,0.7\right)
\end{gathered}
$$

Now, we obtain the following theorem which has the properties of the $3-\mathrm{PF} \beta \mathrm{CRS}$ model. The proof of this theorem is straightforward from Definition 19 and Theorem 1, so, we omit this proof.

Theorem 2 Consider a PF $\beta C A S(\Lambda, \widetilde{\Upsilon})$. Then, we have the following properties

(1) $\mathcal{L}_{3}^{P F}(\mathcal{X})=\left(\mathcal{U}_{3}^{P F}\left(\mathcal{X}^{c}\right)\right)^{c}$.

(2) $\mathcal{U}_{3}^{P F}(\mathcal{X})=\left(\mathcal{L}_{3}^{P F}\left(\mathcal{X}^{c}\right)\right)^{c}$.

(3) If $\mathcal{X} \subseteq \mathcal{Y}$, then $\mathcal{L}_{3}^{P F}(\mathcal{X}) \subseteq \mathcal{L}_{3}^{P F}(\mathcal{Y})$.

(4) If $\mathcal{X} \subseteq \mathcal{Y}$, then $\mathcal{U}_{3}^{P F}(\mathcal{X}) \subseteq \mathcal{U}_{3}^{P F}(\mathcal{Y})$.

(5) $\mathcal{L}_{3}^{P F}(\mathcal{X} \cap \mathcal{Y})=\mathcal{L}_{3}^{P F}(\mathcal{X}) \cap \mathcal{L}_{3}^{P F}(\mathcal{Y})$.

(6) $\mathcal{U}_{3}^{P F}(\mathcal{X} \cap \mathcal{Y}) \subseteq \mathcal{U}_{3}^{P F}(\mathcal{X}) \cap \mathcal{U}_{3}^{P F}(\mathcal{Y})$.

(7) $\mathcal{L}_{3}^{P F}(\mathcal{X} \cup \mathcal{Y}) \supseteq \mathcal{L}_{3}^{P F}(\mathcal{X}) \cup \mathcal{L}_{3}^{P F}(\mathcal{Y})$.

(8) $\mathcal{U}_{3}^{P F}(\mathcal{X} \cup \mathcal{Y})=\mathcal{U}_{3}^{P F}(\mathcal{X}) \cup \mathcal{U}_{3}^{P F}(\mathcal{Y})$. 
Table 4 Result for ${ }_{1} \widetilde{\mathbb{U}}_{u_{s}}^{\beta}, s=1,2, \ldots, 6$

\begin{tabular}{lllllll}
\hline & $u_{1}$ & $u_{2}$ & $u_{3}$ & $u_{4}$ & $u_{5}$ & $u_{6}$ \\
\hline$\widetilde{\mathbb{\Psi}}_{u_{1}}^{\beta}$ & $(0.3,0.8)$ & $(0.6,0.7)$ & $(0.3,0.9)$ & $(0.6,0.7)$ & $(0.2,0.7)$ & $(0.6,0.6)$ \\
$1 \widetilde{\Psi}_{u_{2}}^{\beta}$ & $(0.4,0.8)$ & $(0.3,0.7)$ & $(0.4,0.6)$ & $(0.4,0.4)$ & $(0.2,0.7)$ & $(0.4,0.6)$ \\
$1 \widetilde{\mathbb{\Psi}}_{u_{3}}^{\beta}$ & $(0.4,0.8)$ & $(0.2,0.7)$ & $(0.3,0.7)$ & $(0.4,0.4)$ & $(0.3,0.8)$ & $(0.4,0.5)$ \\
$1 \widetilde{\mathbb{q}}_{u_{4}}^{\beta}$ & $(0.4,0.6)$ & $(0.3,0.7)$ & $(0.4,0.6)$ & $(0.4,0.8)$ & $(0.2,0.7)$ & $(0.4,0.6)$ \\
$1 \widetilde{\Psi}_{u_{5}}^{\beta}$ & $(0.4,0.8)$ & $(0.4,0.5)$ & $(0.6,0.7)$ & $(0.3,0.9)$ & $(0.3,0.7)$ & $(0.4,0.5)$ \\
$1 \widetilde{\Psi}_{u_{6}}^{\beta}$ & $(0.6,0.7)$ & $(0.4,0.7)$ & $(0.3,0.9)$ & $(0.3,0.9)$ & $(0.5,0.7)$ & $(0.2,0.7)$ \\
\hline
\end{tabular}

Definition 20 Consider a $\operatorname{PF} \beta \operatorname{CAS}(\Lambda, \widetilde{\Upsilon})$ and PFN $\beta=$ $\left(\vartheta_{\beta}, \zeta_{\beta}\right)$. Thus the rough and precision degrees of $\mathcal{X} \in$ $\mathrm{PF}(\Lambda)$ are respectively seen as follows.

$$
\begin{gathered}
{ }_{3} \Re_{\tilde{\mathcal{P}}^{\beta}(\mathcal{X})}=\mathcal{D}\left(\mathcal{L}_{3}^{\mathrm{PF}}(\mathcal{X}), \mathcal{U}_{3}^{\mathrm{PF}}(\mathcal{X})\right), \\
{ }_{3} \mathscr{P}_{\hat{\mathcal{P}} \beta(\mathcal{X})}=1-{ }_{3} \Re_{\tilde{\mathcal{P}}^{\beta}(\mathcal{X})} .
\end{gathered}
$$

Example 6 Consider Examples 1 and 2. Then the following results hold.

${ }_{3} \Re_{\tilde{\mathcal{P}}^{\beta}(\mathcal{X})}=0.228,{ }_{3} \mathscr{P}_{\tilde{\mathcal{P}}^{\beta}(\mathcal{X})}=0.772$

\section{The third type of a CPFRS}

Definition 21 Consider a $\operatorname{PF} \beta \operatorname{CAS}(\Lambda, \tilde{\Upsilon})$ and $\operatorname{PFN} \beta=$ $\left(\vartheta_{\beta}, \zeta_{\beta}\right)$. Then for each $u \in \Lambda$, define a new kind of PF $\beta$-neighborhood as follows.

${ }_{2} \widetilde{\mathbb{I}}_{u}^{\beta}=\widetilde{\mathcal{P}}_{u}^{\beta} \vee \hat{\widetilde{\mathcal{P}}}_{u}^{\beta}$.

Example 7 Consider Example 1. We compute ${ }_{2} \widetilde{\mathbb{I}}_{u}^{\beta}$ as follows in Table 5.

Definition 22 Consider a $\operatorname{PF} \beta \operatorname{CAS}(\Lambda, \tilde{\Upsilon})$ and $\operatorname{PFN} \beta=$ $\left(\vartheta_{\beta}, \zeta_{\beta}\right)$. For all $u \in \Lambda$ and $\mathcal{X} \in \operatorname{PF}(\Lambda)$. Define the 4-PFLA $\mathcal{L}_{4}^{\mathrm{PF}}(\mathcal{X})$ and 4-PFUA $\mathcal{U}_{4}^{\mathrm{PF}}(\mathcal{X})$ as follows.

$$
\begin{aligned}
& \mathcal{L}_{4}^{\mathrm{PF}}(\mathcal{X})=\left\{\begin{array}{r}
\left(u_{i}, \wedge_{i=1}^{n}\left(\zeta_{2 \widetilde{\mathbb{N}}_{u}^{\beta}}\left(u_{i}, u_{j}\right) \vee \vartheta_{\mathcal{X}}\left(u_{j}\right)\right),\right. \\
\left.\vee_{i=1}^{n}\left(\vartheta_{2 \widetilde{\mathbb{N}}_{u}^{\beta}}\left(u_{i}, u_{j}\right) \wedge \zeta_{\mathcal{X}}\left(u_{j}\right)\right)\right)
\end{array}\right\}, \\
& \mathcal{U}_{4}^{\mathrm{PF}}(\mathcal{X})=\left\{\begin{array}{r}
\left(u_{i}, \vee_{i=1}^{n}\left(\vartheta_{2 \widetilde{\Psi}_{u}^{\beta}}\left(u_{i}, u_{j}\right) \wedge \vartheta_{\mathcal{X}}\left(u_{j}\right)\right),\right. \\
\left.\wedge_{i=1}^{n}\left(\zeta_{2} \widetilde{\mathbb{H}}_{u}^{\beta}\left(u_{i}, u_{j}\right) \vee \zeta_{\mathcal{X}}\left(u_{j}\right)\right)\right)
\end{array}\right\}
\end{aligned}
$$

Then the pair $\left(\mathcal{L}_{4}^{\mathrm{PF}}(\mathcal{X}), \mathcal{U}_{4}^{\mathrm{PF}}(\mathcal{X})\right)$ is called the 4-PF $\beta$ CRS.

Example 8 Consider Examples 1 and 2. Then the following results hold.

$$
\begin{aligned}
& \mathcal{L}_{4}^{\mathrm{PF}}(\mathcal{X})=\left(u_{1}, 0.6,0.7\right)+\left(u_{2}, 0.4,0.7\right) \\
&+\left(u_{3}, 0.4,0.7\right)+\left(u_{4}, 0.4,0.7\right) \\
&+\left(u_{5}, 0.4,0.6\right)+\left(u_{6}, 0.4,0.7\right)
\end{aligned}
$$

$$
\begin{aligned}
& \mathcal{U}_{4}^{\mathrm{PF}}(\mathcal{X})=\left(u_{1}, 0.8,0.4\right)+\left(u_{2}, 0.7,0.4\right) \\
&+\left(u_{3}, 0.7,0.4\right)+\left(u_{4}, 0.8,0.4\right) \\
&+\left(u_{5}, 0.8,0.3\right)+\left(u_{6}, 0.8,0.2\right)
\end{aligned}
$$

Theorem 3 Consider a PF $\beta C A S(\Lambda, \widetilde{\Upsilon})$. Then, we have the following properties

(1) $\mathcal{L}_{4}^{P F}(\mathcal{X})=\left(\mathcal{U}_{4}^{P F}\left(\mathcal{X}^{c}\right)\right)^{c}$.

(2) $\mathcal{U}_{4}^{P F}(\mathcal{X})=\left(\mathcal{L}_{4}^{P F}\left(\mathcal{X}^{c}\right)\right)^{c}$.

(3) If $\mathcal{X} \subseteq \mathcal{Y}$, then $\mathcal{L}_{4}^{P F}(\mathcal{X}) \subseteq \mathcal{L}_{4}^{P F}(\mathcal{Y})$.

(4) If $\mathcal{X} \subseteq \mathcal{Y}$, then $\mathcal{U}_{4}^{P F}(\mathcal{X}) \subseteq \mathcal{U}_{4}^{P F}(\mathcal{Y})$.

(5) $\mathcal{L}_{4}^{P F}(\mathcal{X} \cap \mathcal{Y})=\mathcal{L}_{4}^{P F}(\mathcal{X}) \cap \mathcal{L}_{4}^{P F}(\mathcal{Y})$.

(6) $\mathcal{U}_{4}^{P F}(\mathcal{X} \cap \mathcal{Y}) \subseteq \mathcal{U}_{4}^{P F}(\mathcal{X}) \cap \mathcal{U}_{4}^{P F}(\mathcal{Y})$.

(7) $\mathcal{L}_{4}^{P F}(\mathcal{X} \cup \mathcal{Y}) \supseteq \mathcal{L}_{4}^{P F}(\mathcal{X}) \cup \mathcal{L}_{4}^{P F}(\mathcal{Y})$.

(8) $\mathcal{U}_{4}^{P F}(\mathcal{X} \cup \mathcal{Y})=\mathcal{U}_{4}^{P F}(\mathcal{X}) \cup \mathcal{U}_{4}^{P F}(\mathcal{Y})$.

Proof The proof is similar to Theorem 1 using Definition 22.

Definition 23 Consider a $\operatorname{PF} \beta \operatorname{CAS}(\Lambda, \tilde{\Upsilon})$ and $\operatorname{PFN} \beta=$ $\left(\vartheta_{\beta}, \zeta_{\beta}\right)$. Thus the rough and precision degrees of $\mathcal{X} \in$ $\operatorname{PF}(\Lambda)$ are respectively seen as follows.

$$
\begin{gathered}
{ }_{4} \Re_{\tilde{\mathcal{P}}^{\beta}(\mathcal{X})}=\mathcal{D}\left(\mathcal{L}_{4}^{\mathrm{PF}}(\mathcal{X}), \mathcal{U}_{4}^{\mathrm{PF}}(\mathcal{X})\right), \\
{ }_{4} \mathscr{P}_{\hat{\mathcal{P}}^{\beta}(\mathcal{X})}=1-{ }_{4} \Re_{\tilde{\mathcal{P}}^{\beta}(\mathcal{X})} .
\end{gathered}
$$

Example 9 Consider Examples 1 and 2. Then the following results hold.

${ }_{4} \Re_{\tilde{\mathcal{P}}^{\beta}(\mathcal{X})}=0.40195,{ }_{4} \mathscr{P}_{\tilde{\mathcal{P}}^{\beta}(\mathcal{X})}=0.59805$

\section{Relationships between the proposed methods}

Below, we proceed to explain some relationships among these kinds.

Proposition 1 Consider a PF $\beta C A S(\Lambda, \widetilde{\Upsilon})$ and $\mathcal{X} \in \operatorname{PF}(\Lambda)$. Then the following properties holds. 
Table 5 Result for ${ }_{2} \widetilde{\mathbb{U}}_{u_{s}}^{\beta}, s=1,2, \ldots, 6$

\begin{tabular}{lllllll}
\hline & $u_{1}$ & $u_{2}$ & $u_{3}$ & $u_{4}$ & $u_{5}$ & $u_{6}$ \\
\hline${ }_{2} \widetilde{\mathbb{I}}_{u_{1}}^{\beta}$ & $(0.8,0.3)$ & $(0.7,0.6)$ & $(0.9,0.3)$ & $(0.7,0.6)$ & $(0.7,0.2)$ & $(0.6,0.6)$ \\
$2 \widetilde{\mathbb{I}}_{u_{2}}^{\beta}$ & $(0.8,0.4)$ & $(0.7,0.3)$ & $(0.6,0.4)$ & $(0.4,0.4)$ & $(0.7,0.2)$ & $(0.6,0.4)$ \\
$2 \widetilde{\mathbb{T}}_{u_{3}}^{\beta}$ & $(0.8,0.4)$ & $(0.7,0.2)$ & $(0.7,0.3)$ & $(0.4,0.4)$ & $(0.8,0.3)$ & $(0.5,0.4)$ \\
${ }_{2} \widetilde{\mathbb{I}}_{u_{4}}^{\beta}$ & $(0.6,0.4)$ & $(0.7,0.3)$ & $(0.6,0.4)$ & $(0.8,0.4)$ & $(0.7,0.2)$ & $(0.6,0.4)$ \\
${ }_{2} \widetilde{\mathbb{I}}_{u_{5}}^{\beta}$ & $(0.8,0.4)$ & $(0.5,0.4)$ & $(0.7,0.6)$ & $(0.9,0.3)$ & $(0.7,0.3)$ & $(0.5,0.4)$ \\
$2 \widetilde{\mathbb{T}}_{u_{6}}^{\beta}$ & $(0.7,0.6)$ & $(0.7,0.4)$ & $(0.9,0.3)$ & $(0.9,0.3)$ & $(0.7,0.5)$ & $(0.7,0.2)$ \\
\hline
\end{tabular}

(1) $\mathcal{L}_{4}^{P F}(\mathcal{X}) \leq \mathcal{L}_{2}^{P F}(\mathcal{X}) \leq \mathcal{L}_{3}^{P F}(\mathcal{X})$.

(2) $\mathcal{L}_{4}^{P F}(\mathcal{X}) \leq \mathcal{L}_{1}^{P F}(\mathcal{X}) \leq \mathcal{L}_{3}^{P F}(\mathcal{X})$.

(3) $\mathcal{U}_{3}^{P F}(\mathcal{X}) \leq \mathcal{U}_{2}^{P F}(\mathcal{X}) \leq \mathcal{U}_{4}^{P F}(\mathcal{X})$.

(4) $\mathcal{U}_{3}^{P F}(\mathcal{X}) \leq \mathcal{U}_{1}^{P F}(\mathcal{X}) \leq \mathcal{U}_{4}^{P F}(\mathcal{X})$

Proof It is clear from Definitions 7, 16, 19 and 22.

Proposition 2 Consider a PF $\beta C A S(\Lambda, \widetilde{\Upsilon})$ and $\mathcal{X} \in P F(\Lambda)$. Then the following properties holds.

(1) $\mathcal{L}_{3}^{P F}(\mathcal{X}) \geq \mathcal{L}_{1}^{P F}(\mathcal{X}) \cup \mathcal{L}_{2}^{P F}(\mathcal{X})$.

(2) $\mathcal{U}_{3}^{P F}(\mathcal{X}) \leq \mathcal{U}_{1}^{P F}(\mathcal{X}) \cap \mathcal{U}_{2}^{P F}(\mathcal{X})$.

(3) $\mathcal{L}_{4}^{P F}(\mathcal{X}) \leq \mathcal{L}_{1}^{P F}(\mathcal{X}) \cap \mathcal{L}_{2}^{P F}(\mathcal{X})$.

(4) $\mathcal{U}_{4}^{P F}(\mathcal{X}) \geq \mathcal{U}_{1}^{P F}(\mathcal{X}) \cup \mathcal{U}_{2}^{P F}(\mathcal{X})$

Proof It is obvious.

Remark 1 Consider a $\operatorname{PF} \beta \operatorname{CAS}(\Lambda, \tilde{\Upsilon})$ and $\mathcal{X} \in \operatorname{PF}(\Lambda)$. According to Example 2, you can see the following relations.

(1) $\mathcal{L}_{1}^{\mathrm{PF}}(\mathcal{X}) \not \leq \mathcal{L}_{2}^{\mathrm{PF}}(\mathcal{X})$ and $\mathcal{L}_{2}^{\mathrm{PF}}(\mathcal{X}) \not \leq \mathcal{L}_{1}^{\mathrm{PF}}(\mathcal{X})$.

(2) $\mathcal{U}_{1}^{\mathrm{PF}}(\mathcal{X}) \not \mathcal{U}_{2}^{\mathrm{PF}}(\mathcal{X})$ and $\mathcal{U}_{2}^{\mathrm{PF}}(\mathcal{X}) \not \mathcal{U}_{1}^{\mathrm{PF}}(\mathcal{X})$.

Based on the above remark and Example 2, the two model 1-PF $\beta$ CRS and 2-PF $\beta$ CRS are distinct from some of them.

\section{q-ROF complementary $\beta$-neighborhood and three novel kinds of Cq-ROFRS}

To treat the insufficiency in PF, Yager's set the notion of qROF. In this section, we define the q-ROF complementary $\beta$-neighborhood and then we present three models of CqROFRS. In addition, we study the relationships between us and the last method by Hussian et al. [56].

\section{q-ROF complementary $\beta$-neighborhood}

Definition 24 Consider a q-ROF $\beta \operatorname{CAS}(\Theta, \widetilde{\Re})$ and q-ROF $\beta=\left(\mu_{\beta}, v_{\beta}\right)$. Then for each $u \in \Theta$, define the q-ROF complementary $\beta$-neighborhood of $u$ as follows.

$\hat{\widetilde{\mathcal{Q}}}_{u}^{\beta}(v)=\widetilde{\mathcal{Q}}_{v}^{\beta}(u), \quad \forall v \in \Theta$.

Example 10 Consider a q-ROF $\beta \operatorname{CAS}(\Theta, \widetilde{\Re}), \Theta=\left\{u_{1}, u_{2}\right.$, $\left.u_{3}, u_{4}, u_{5}, u_{6}\right\}$ and $\widetilde{\mathcal{R}}=\left\{\widetilde{\mathcal{R}}_{1}, \widetilde{\mathcal{R}}_{2}, \widetilde{\mathcal{R}}_{3}, \widetilde{\mathcal{R}}_{4}, \widetilde{\mathcal{R}}_{5}\right\}$, where $\beta=$ $\langle 0.5,0.3,0.8\rangle$ are summarized in Table 6.

It is computed that

$\widetilde{\mathcal{Q}}_{u_{1}}^{(0.8,0.7)}=\widetilde{\mathcal{R}}_{1} \cap \widetilde{\mathcal{R}}_{2} \cap \widetilde{\mathcal{R}}_{5}$

$\widetilde{\mathcal{Q}}_{u_{2}}^{(0.8,0.7)}=\widetilde{\mathcal{R}}_{1} \cap \widetilde{\mathcal{R}}_{2}$,

$\widetilde{\mathcal{Q}}_{u_{3}}^{(0.8,0.7)}=\widetilde{\mathcal{R}}_{1} \cap \widetilde{\mathcal{R}}_{3}$,

$\widetilde{\mathcal{Q}}_{u_{4}}^{(0.8,0.7)}=\widetilde{\mathcal{R}}_{1} \cap \widetilde{\mathcal{R}}_{4} \cap \widetilde{\mathcal{R}}_{5}$,

$\widetilde{\mathcal{Q}}_{u_{5}}^{(0.8,0.7)}=\widetilde{\mathcal{R}}_{2} \cap \widetilde{\mathcal{R}}_{4}$,

$\widetilde{\mathcal{Q}}_{u_{6}}^{(0.8,0.7)}=\widetilde{\mathcal{R}}_{1} \cap \widetilde{\mathcal{R}}_{3}$

and the complete values of $\widetilde{\mathcal{Q}}_{u}^{(0.8,0.7)}$ are listed in Table 7.

Thus, we can obtain their complement values $\widetilde{\mathcal{Q}}_{u}^{(0 . \hat{8}, 0.7)}$ as in Table 8.

\section{The first type of a Cq-ROFRS}

Definition 25 Consider a q-ROF $\beta$ CAS $(\Theta, \widetilde{\Re})$ and $\beta=\left(\mu_{\beta}, v_{\beta}\right)$. For all $u \in \Theta$ and $\mathcal{X} \in q-R O F(\Theta)$. Define the 2-q-ROFLA $\mathcal{L}_{2}^{q-R O F}(\mathcal{X})$ and 2-q-ROFUA $\mathcal{U}_{2}^{q-R O F}(\mathcal{X})$ as follows.

$$
\mathcal{L}_{2}^{q-R O F}(\mathcal{X})=\left\{\begin{array}{c}
\left(u_{k}, \wedge_{k=1}^{n}\left(\mu{ }_{\hat{\mathcal{Q}}_{u_{k}}^{\beta}}\left(u_{k}, u_{r}\right) \vee \mu \mathcal{X}\left(u_{r}\right)\right),\right. \\
\left.\vee_{k=1}^{n}\left(v_{\tilde{\mathcal{Q}}_{u_{k}}^{\beta}}\left(u_{k}, u_{r}\right) \wedge v_{\mathcal{X}}\left(u_{r}\right)\right)\right)_{q}
\end{array}\right\}
$$

$\mathcal{U}_{2}^{q-R O F}(\mathcal{X})=\left\{\begin{array}{c}\left(u_{k}, \vee_{k=1}^{n}\left(\mu_{\tilde{\mathcal{Q}}_{u_{k}}^{\beta}}\left(u_{k}, u_{r}\right) \wedge \mu \mathcal{X}\left(u_{r}\right)\right),\right. \\ \left.\wedge_{k=1}^{n}\left(v_{\tilde{\mathcal{Q}}_{u_{k}}^{\beta}}\left(u_{k}, u_{r}\right) \vee v_{\mathcal{X}}\left(u_{r}\right)\right)\right)_{q}\end{array}\right\}$

Then the pair $\left(\mathcal{L}_{2}^{q-R O F}(\mathcal{X}), \mathcal{U}_{2}^{q-R O F}(\mathcal{X})\right)$ is called the 2-q-ROF $\beta$ CRS. 
Table 6 Result for $(\Theta, \widetilde{\Re})$

\begin{tabular}{llllll}
\hline & $\widetilde{\mathcal{R}}_{1}$ & $\widetilde{\mathcal{R}}_{2}$ & $\widetilde{\mathcal{R}}_{3}$ & $\widetilde{\mathcal{R}}_{4}$ & $\widetilde{\mathcal{R}}_{5}$ \\
\hline$u_{1}$ & $(0.9,0.5)$ & $(0.85,0.65)$ & $(0.7,0.8)$ & $(0.5,0.9)$ & $(0.9,0.3)$ \\
$u_{2}$ & $(0.89,0.7)$ & $(0.93,0.45)$ & $(0.79,0.65)$ & $(0.69,0.8)$ & $(0.65,0.95)$ \\
$u_{3}$ & $(0.95,0.6)$ & $(0.69,0.85)$ & $(0.98,0.63)$ & $(0.7,0.4)$ & $(0.5,0.9)$ \\
$u_{4}$ & $(0.85,0.7)$ & $(0.6,0.9)$ & $(0.55,0.85)$ & $(0.97,0.3)$ & $(0.89,0.4)$ \\
$u_{5}$ & $(0.6,0.87)$ & $(0.9,0.45)$ & $(0.69,0.85)$ & $(0.92,0.6)$ & $(0.3,0.75)$ \\
$u_{6}$ & $(0.88,0.55)$ & $(0.6,0.9)$ & $(0.9,0.63)$ & $(0.8,0.7)$ & $(0.5,0.89)$ \\
\hline
\end{tabular}

Table 7 Result for $\widetilde{\mathcal{Q}}_{u_{k}}^{(0.8,0.7)}, k=1,2, \ldots, 6$

\begin{tabular}{lllllll}
\hline & $u_{1}$ & $u_{2}$ & $u_{3}$ & $u_{4}$ & $u_{5}$ & $u_{6}$ \\
\hline$\widetilde{\mathcal{Q}}_{u_{1}}^{\beta}$ & $(0.35,0.65)$ & $(0.35,0.65)$ & $(0.7,0.8)$ & $(0.5,0.9)$ & $(0.5,0.9)$ & $(0.7,0.8)$ \\
$\widetilde{\mathcal{Q}}_{u_{2}}^{\beta}$ & $(0.65,0.95)$ & $(0.39,0.7)$ & $(0.79,0.7)$ & $(0.65,0.93)$ & $(0.69,0.3)$ & $(0.79,0.7)$ \\
$\widetilde{\mathcal{Q}}_{u_{3}}^{\beta}$ & $(0.5,0.9)$ & $(0.69,0.35)$ & $(0.95,0.6)$ & $(0.5,0.9)$ & $(0.69,0.85)$ & $(0.95,0.6)$ \\
$\widetilde{\mathcal{Q}}_{u_{4}}^{\beta}$ & $(0.6,0.9)$ & $(0.6,0.9)$ & $(0.55,0.85)$ & $(0.85,0.7)$ & $(0.92,0.6)$ & $(0.55,0.85)$ \\
$\widetilde{\mathcal{Q}}_{u_{5}}^{\beta}$ & $(0.6,0.87)$ & $(0.6,0.87)$ & $(0.6,0.87)$ & $(0.6,0.87)$ & $(0.6,0.9)$ & $(0.6,0.8)$ \\
$\widetilde{\mathcal{Q}}_{u_{6}}^{\beta}$ & $(0.5,0.9)$ & $(0.6,0.9)$ & $(0.88,0.63)$ & $(0.5,0.89)$ & $(0.6,0.9)$ & $(0.88,0.63)$ \\
\hline
\end{tabular}

Table 8 Result for $\widetilde{\mathcal{Q}}_{u_{k}}^{(0 . \hat{8}, 0.7)}, k=1,2, \ldots, 6$

\begin{tabular}{|c|c|c|c|c|c|c|}
\hline & $u_{1}$ & $u_{2}$ & $u_{3}$ & $u_{4}$ & $u_{5}$ & $u_{6}$ \\
\hline$\tilde{\widetilde{\mathcal{Q}}}_{u_{1}}^{\beta}$ & $(0.65,0.35)$ & $(0.65,0.35)$ & $(0.8,0.7)$ & $(0.9,0.5)$ & $(0.9,0.5)$ & $(0.8,0.7)$ \\
\hline$\tilde{\mathcal{Q}}_{u_{2}}^{\beta}$ & $(0.95,0.65)$ & $(0.7,0.39)$ & $(0.7,0.79)$ & $(0.93,0.65)$ & $(0.3,0.69)$ & $(0.7,0.79)$ \\
\hline$\hat{\widetilde{\mathcal{Q}}_{u_{3}}^{\beta}}$ & $(0.9,0.5)$ & $(0.35,0.69)$ & $(0.6,0.95)$ & $(0.9,0.5)$ & $(0.85,0.69)$ & $(0.6,0.95)$ \\
\hline$\hat{\mathcal{Q}}_{u_{4}}^{\beta}$ & $(0.9,0.6)$ & $(0.9,0.6)$ & $(0.85,0.55)$ & $(0.7,0.85)$ & $(0.6,0.92)$ & $(0.85,0.55)$ \\
\hline$\tilde{\widetilde{\mathcal{Q}}}_{u_{5}}^{\beta}$ & $(0.87,0.6)$ & $(0.87,0.6)$ & $(0.87,0.6)$ & $(0.87,0.6)$ & $(0.9,0.6)$ & $(0.8,0.6)$ \\
\hline$\tilde{\widetilde{\mathcal{Q}}}_{u_{6}}^{\beta}$ & $(0.9,0.5)$ & $(0.9,0.6)$ & $(0.63,0.88)$ & $(0.89,0.5)$ & $(0.9,0.6)$ & $(0.63,0.88)$ \\
\hline
\end{tabular}

Example 11 Consider Example 10 and

$$
\mathcal{X}=\left\{\begin{array}{l}
\left(u_{1}, 0.91,0.62\right),\left(u_{2}, 0.58,0.83\right),\left(u_{3}, 0.8,0.75\right), \\
\left(u_{4}, 0.95,0.35\right),\left(u_{5}, 0.8,0.7\right),\left(u_{6}, 0.98,0.37\right)
\end{array}\right\}
$$

Then we get the following results.

$\begin{aligned} \text { (1) } \mathcal{L}_{1}^{\mathrm{q}-\mathrm{ROF}}(\mathcal{X}) & =\left\{\begin{array}{l}\left(u_{1}, 0.35,0.9\right),\left(u_{2}, 0.39,0.95\right), \\ \left(u_{3}, 0.5,0.9\right),\left(u_{4}, 0.55,0.9\right), \\ \left(u_{5}, 0.58,0.9\right),\left(u_{6}, 0.5,0.9\right)\end{array}\right\}, \\ \mathcal{U}_{1}^{\mathrm{q}-\mathrm{ROF}}(\mathcal{X}) & =\left\{\begin{array}{l}\left(u_{1}, 0.98,0.35\right),\left(u_{2}, 0.98,0.3\right), \\ \left(u_{3}, 0.98,0.35\right),\left(u_{4}, 0.98,0.35\right), \\ \left(u_{5}, 0.98,0.35\right),\left(u_{6}, 0.98,0.35\right)\end{array}\right\} .\end{aligned}$

$$
\begin{aligned}
\text { (2) } \mathcal{L}_{2}^{\mathrm{q}-\mathrm{ROF}}(\mathcal{X}) & =\left\{\begin{array}{l}
\left(u_{1}, 0.58,0.83\right),\left(u_{2}, 0.3,0.83\right), \\
\left(u_{3}, 0.35,0.95\right),\left(u_{4}, 0.58,0.92\right), \\
\left(u_{5}, 0.58,0.83\right),\left(u_{6}, 0.58,0.88\right)
\end{array}\right\}, \\
\mathcal{U}_{2}^{\mathrm{q}-\mathrm{ROF}}(\mathcal{X}) & =\left\{\begin{array}{c}
\left(u_{1}, 0.98,0.35\right),\left(u_{2}, 0.98,0.35\right), \\
\left(u_{3}, 0.98,0.35\right),\left(u_{4}, 0.98,0.35\right), \\
\left(u_{5}, 0.98,0.35\right),\left(u_{6}, 0.98,0.35\right)
\end{array}\right\} .
\end{aligned}
$$

Theorem 4 Consider a $q$-ROF $\beta C A S(\Theta, \widetilde{\Re})$. Then, we have the following properties

(1) $\mathcal{L}_{2}^{q-R O F}(\mathcal{X})=\left(\mathcal{U}_{2}^{q-R O F}\left(\mathcal{X}^{c}\right)\right)^{c}$.

(2) $\mathcal{U}_{2}^{q-R O F}(\mathcal{X})=\left(\mathcal{L}_{2}^{q-R O F}\left(\mathcal{X}^{c}\right)\right)^{c}$.

(3) If $\mathcal{X} \subseteq \mathcal{Y}$, then $\mathcal{L}_{2}^{q-R O F}(\mathcal{X}) \subseteq \mathcal{L}_{2}^{q-R O F}(\mathcal{Y})$.

(4) If $\mathcal{X} \subseteq \mathcal{Y}$, then $\mathcal{U}_{2}^{q-R O F}(\mathcal{X}) \subseteq \mathcal{U}_{2}^{q-R O F}(\mathcal{Y})$.

(5) $\mathcal{L}_{2}^{q-R O F}(\mathcal{X} \cap \mathcal{Y})=\mathcal{L}_{2}^{q-R O F}(\mathcal{X}) \cap \mathcal{L}_{2}^{q-R O F}(\mathcal{Y})$ 
(6) $\mathcal{U}_{2}^{q-R O F}(\mathcal{X} \cap \mathcal{Y}) \subseteq \mathcal{U}_{2}^{q-R O F}(\mathcal{X}) \cap \mathcal{U}_{2}^{q-R O F}(\mathcal{Y})$

(7) $\mathcal{L}_{2}^{q-R O F}(\mathcal{X} \cup \mathcal{Y}) \supseteq \mathcal{L}_{2}^{q-R O F}(\mathcal{X}) \cup \mathcal{L}_{2}^{q-R O F}(\mathcal{Y})$.

(8) $\mathcal{U}_{2}^{q-R O F}(\mathcal{X} \cup \mathcal{Y})=\mathcal{U}_{2}^{q-R O F}(\mathcal{X}) \cup \mathcal{U}_{2}^{q-R O F}(\mathcal{Y})$.

(9) $\mathcal{L}_{2}^{q-R O F}(\mathcal{X}) \subseteq \mathcal{X} \subseteq \mathcal{U}_{2}^{q-R O F}(\mathcal{X})$.

Proof of Theorem 4: We prove (1), (3), (5), (7) and (9) only.

(1)

$$
\begin{aligned}
& \mathcal{L}_{2}^{q-R O F}\left(\mathcal{X}^{c}\right) \\
& =\left\{\begin{array}{c}
\left(u_{k}, \wedge_{k=1}^{n}\left(\mu_{\tilde{\mathcal{Q}}_{u_{k}}^{\beta}}\left(u_{k}, u_{r}\right) \vee \mu \mathcal{X}^{c}\left(u_{r}\right)\right),\right. \\
\left.\vee_{k=1}^{n}\left(v_{\hat{\mathcal{Q}}_{u_{k}}^{\beta}}\left(u_{k}, u_{r}\right) \wedge v_{\mathcal{X}^{c}}\left(u_{r}\right)\right)\right)_{q}
\end{array}\right\} \\
& =\left\{\begin{array}{c}
\left(u_{k}, \wedge_{k=1}^{n}\left(\mu_{\hat{\mathcal{Q}}_{u_{k}}^{\beta}}\left(u_{k}, u_{r}\right) \vee\left(1-\mu_{\mathcal{X}}\left(u_{j}\right)\right)\right),\right. \\
\left.\vee_{k=1}^{n}\left(v_{\tilde{\mathcal{Q}}_{u_{k}}^{\beta}}\left(u_{k}, u_{r}\right) \wedge\left(1-v_{\mathcal{X}}\left(u_{r}\right)\right)\right)\right)_{q}
\end{array}\right\} \\
& =\left(\mathcal{U}_{2}^{q-R O F}(\mathcal{X})\right)^{c}
\end{aligned}
$$

(3) Let $\mathcal{X}, \mathcal{Y} \in \mathrm{q}-\operatorname{ROF}(\Theta)$ such that $\mathcal{X} \subseteq \mathcal{Y}$ (i.e., $\mu_{\mathcal{X}} \leq \preceq \mathcal{Y}$ and $\left.v_{\mathcal{X}} \geq v_{\mathcal{Y}}\right)$ and $u \in \Theta$. Then we get the following result.

$$
\begin{aligned}
& \mathcal{L}_{2}^{q-R O F}(\mathcal{X})(u) \\
& \quad=\left\{\begin{array}{r}
\left(u_{k}, \wedge_{k=1}^{n}\left(\mu_{\tilde{\mathcal{Q}}_{u_{k}}^{\beta}}\left(u_{k}, u_{r}\right) \vee \mu_{\mathcal{X}}\left(u_{r}\right)\right),\right. \\
\left.\vee_{k=1}^{n}\left(v_{\hat{\mathcal{Q}}_{u_{k}}^{\beta}}\left(u_{k}, u_{r}\right) \wedge v_{\mathcal{X}}\left(u_{r}\right)\right)\right)_{q}
\end{array}\right\}
\end{aligned}
$$

Now for $\mathcal{X} \subseteq \mathcal{Y}$, we have

$$
\begin{aligned}
\mu_{\mathcal{L}_{2}^{q-R O F}(\mathcal{X})} & =\wedge_{k=1}^{n}\left(\mu_{\tilde{\mathcal{Q}}_{u_{k}}^{\beta}}\left(u_{k}, u_{r}\right) \vee \mu \mathcal{X}\left(u_{r}\right)\right) \\
& \leq \wedge_{k=1}^{n}\left(\mu_{\hat{\mathcal{Q}}_{u_{k}}^{\beta}}\left(u_{k}, u_{r}\right) \vee \mu \mathcal{Y}\left(u_{r}\right)\right) \\
& =\mu_{\mathcal{L}_{2}^{q-R O F}(\mathcal{Y})}
\end{aligned}
$$

and

$$
\begin{aligned}
{ }^{\nu_{\mathcal{L}_{2}}^{q-R O F}(\mathcal{X})} & =\vee_{k=1}^{n}\left(\mu_{\tilde{\mathcal{Q}}_{u_{k}}^{\beta}}\left(u_{k}, u_{r}\right) \wedge v_{\mathcal{X}}\left(u_{r}\right)\right) \\
& \geq \vee_{k=1}^{n}\left(\mu_{\hat{\mathcal{Q}}_{u_{k}}^{\beta}}\left(u_{k}, u_{r}\right) \wedge v_{\mathcal{Y}}\left(u_{r}\right)\right) \\
& =v_{\mathcal{L}_{2}^{q-R O F}(\mathcal{Y})}
\end{aligned}
$$

Therefore, $\mathcal{L}_{2}^{q-R O F}(\mathcal{X}) \subseteq \mathcal{L}_{2}^{q-R O F}(\mathcal{Y})$.

(5)

$$
\begin{aligned}
& \mathcal{L}_{2}^{q-R O F}(\mathcal{X} \cap \mathcal{Y}) \\
& =\left\{\begin{array}{c}
\left(u_{k}, \wedge_{k=1}^{n}\left(\mu_{\hat{\mathcal{Q}}_{u_{k}}^{\beta}}\left(u_{k}, u_{r}\right) \vee \mu_{(\mathcal{X} \cap \mathcal{Y})}\left(u_{r}\right)\right),\right. \\
\left.\vee_{k=1}^{n}\left(v_{\tilde{\mathcal{Q}}_{u_{k}}^{\beta}}\left(u_{k}, u_{r}\right) \wedge v_{(\mathcal{X} \cap \mathcal{Y})}\left(u_{r}\right)\right)\right)_{q}
\end{array}\right\} \\
& =\left\{\begin{array}{r}
\left(u_{k}, \wedge_{k=1}^{n}\left(\mu_{\tilde{\mathcal{Q}}_{u_{k}}^{\beta}}\left(u_{k}, u_{r}\right) \vee(\mu \mathcal{X}(y) \cap \mu \mathcal{Y}(y))\right),\right. \\
\left.\vee_{r=1}^{m}\left(v_{\hat{\mathcal{Q}}_{u_{k}}^{\beta}}\left(u_{k}, u_{r}\right) \wedge\left(v_{\mathcal{X}}(y) \cap \mathcal{v}_{\mathcal{Y}}(y)\right)\right)\right)
\end{array}\right\}
\end{aligned}
$$

$$
\begin{aligned}
& =\left\{\begin{array}{r}
\left(u_{k}, \wedge_{k=1}^{n}\left(\mu_{\tilde{\mathcal{Q}}_{u_{k}}^{\beta}}\left(u_{k}, u_{r}\right) \vee \mu \mathcal{X}(y)\right)\right. \\
\wedge_{k=1}^{n}\left(\mu_{\tilde{\mathcal{Q}}_{u_{k}}^{\beta}}\left(u_{k}, u_{r}\right) \vee \mu \mathcal{Y}(y)\right), \\
\vee_{r=1}^{m}\left(v_{\hat{\mathcal{Q}}_{u_{k}}^{\beta}}\left(u_{k}, u_{r}\right) \wedge v_{\mathcal{X}}(y)\right) \\
\left.\vee_{r=1}^{m}\left(v_{\tilde{\mathcal{Q}}_{u_{k}}^{\beta}}\left(u_{k}, u_{r}\right) \wedge v_{\mathcal{Y}}(y)\right)\right)
\end{array}\right\} \\
& =\mathcal{L}_{2}^{q-R O F}(\mathcal{X}) \cap \mathcal{L}_{2}^{q-R O F}(\mathcal{Y}) \\
& \mathcal{L}_{2}^{q-R O F}(\mathcal{X} \cup \mathcal{Y}) \\
& =\left\{\begin{array}{c}
\left(u_{k}, \wedge_{k=1}^{n}\left(\mu_{\tilde{\mathcal{Q}}_{u_{k}}^{\beta}}\left(u_{k}, u_{r}\right) \vee \mu_{(\mathcal{X} \cup \mathcal{Y})}\left(u_{r}\right)\right),\right. \\
\left.\vee_{k=1}^{n}\left(v_{\tilde{\mathcal{Q}}_{u_{k}}^{\beta}}\left(u_{k}, u_{r}\right) \wedge v_{(\mathcal{X} \cup \mathcal{Y})}\left(u_{r}\right)\right)\right)_{q}
\end{array}\right\} \\
& =\left\{\begin{array}{r}
\left(u_{k}, \wedge_{k=1}^{n}\left(\mu_{\tilde{\mathcal{Q}}_{u_{k}}^{\beta}}\left(u_{k}, u_{r}\right) \wedge(\mu \mathcal{X}(y) \cup \mu \mathcal{Y}(y))\right),\right. \\
\left.\vee_{r=1}^{m}\left(v_{\hat{\mathcal{Q}}_{u_{k}}^{\beta}}\left(u_{k}, u_{r}\right) \wedge\left(\nu_{\mathcal{X}}(y) \cup v_{\mathcal{Y}}(y)\right)\right)\right)
\end{array}\right\} \\
& \geq\left\{\begin{array}{r}
\left(u_{k}, \wedge_{k=1}^{n}\left(\mu_{\tilde{\mathcal{Q}}_{u_{k}}^{\beta}}\left(u_{k}, u_{r}\right) \vee \mu \mathcal{X}(y)\right)\right. \\
\vee \wedge_{k=1}^{n}\left(\mu_{\tilde{\mathcal{Q}}_{u_{k}}^{\beta}}\left(u_{k}, u_{r}\right) \vee \mu \mathcal{Y}(y)\right), \\
\vee_{r=1}^{m}\left(v_{\hat{\mathcal{Q}}_{u_{k}}^{\beta}}\left(u_{k}, u_{r}\right) \wedge v_{\mathcal{X}}(y)\right) \\
\left.\wedge \vee_{r=1}^{m}\left(v_{\tilde{\mathcal{Q}}_{u_{k}}^{\beta}}\left(u_{k}, u_{r}\right) \wedge v_{\mathcal{Y}}(y)\right)\right)
\end{array}\right\} \\
& =\mathcal{L}_{2}^{q-R O F}(\mathcal{X}) \cup \mathcal{L}_{2}^{q-R O F}(\mathcal{Y})
\end{aligned}
$$

(9) Straightforward.

Definition 26 Consider a q-ROF $\beta \operatorname{CAS}(\Theta, \widetilde{\Re})$ and $\beta=$ $\left(\mu_{\beta}, v_{\beta}\right)$. Thus the rough and precision degrees of $\mathcal{X} \in$ $\mathrm{q}-\operatorname{ROF}(\Theta)$ are respectively seen as follows.

$$
\begin{aligned}
& { }_{2} \Re_{\widetilde{\mathcal{Q}}^{\beta}(\mathcal{X})}=D\left(\mathcal{L}_{2}^{\mathrm{q}-\mathrm{ROF}}(\mathcal{X}), \mathcal{U}_{2}^{\mathrm{q}-\mathrm{ROF}}(\mathcal{X})\right), \\
& { }_{2} \mathscr{P}_{\hat{\mathcal{Q}}^{\beta}(\mathcal{X})}=1-{ }_{2} \Re_{\hat{\mathcal{Q}}^{\beta}(\mathcal{X})} .
\end{aligned}
$$

Example 12 Consider Example 11, then we have the following outcomes.

$$
\begin{aligned}
& { }_{1} \Re_{\widetilde{\mathcal{Q}}^{\beta}(\mathcal{X})}=0.218, \quad{ }_{1} \mathscr{P}_{\hat{\mathcal{Q}}^{\beta}(\mathcal{X})}=0.782 \\
& { }_{2} \Re_{\widetilde{\mathcal{Q}}^{\beta}(\mathcal{X})}=0.201, \quad 2 \mathscr{P}_{\hat{\mathcal{Q}}^{\beta}(\mathcal{X})}=0.799
\end{aligned}
$$

\section{The second type of a Cq-ROFRS}

Definition 27 Consider a $\mathrm{q}-\operatorname{ROF} \beta \operatorname{CAS}(\Theta, \widetilde{\Re})$ and $\beta=$ $\left(\mu_{\beta}, v_{\beta}\right)$. Then for each $u \in \Theta$, define a new kind of q-ROF $\beta$-neighborhood as follows.

${ }_{1} \widetilde{\mathbb{Q}}_{u}^{\beta}=\widetilde{\mathcal{Q}}_{u}^{\beta} \wedge \hat{\widetilde{\mathcal{Q}}}_{u}^{\beta}$ 
Example 13 Consider Example 10 and we can obtain the following results for $\widetilde{\mathbb{Q}}_{u}^{\beta}$ as listed in Table 9.

Definition 28 Consider a q-ROF $\beta \operatorname{CAS}(\Theta, \widetilde{\Re})$ and $\beta=\left(\mu_{\beta}, v_{\beta}\right)$. For all $u \in \Theta$ and $\mathcal{X} \in q-\operatorname{ROF}(\Theta)$. Define the 3-q-ROFLA $\mathcal{L}_{3}^{q-R O F}(\mathcal{X})$ and 3-q-ROFUA $\mathcal{U}_{3}^{q-R O F}(\mathcal{X})$ as follows.

$\mathcal{L}_{3}^{q-R O F}(\mathcal{X})=\left\{\begin{array}{c}\left(u_{k}, \wedge_{k=1}^{n}\left(\mu_{1} \widetilde{\mathbb{Q}}_{u_{k}}^{\beta}\left(u_{k}, u_{r}\right) \vee \mu_{\mathcal{X}}\left(u_{r}\right)\right),\right. \\ \left.\vee_{k=1}^{n}\left(v_{1} \widetilde{\mathbb{Q}}_{u_{k}}^{\beta}\left(u_{k}, u_{r}\right) \vee v_{\mathcal{X}}\left(u_{r}\right)\right)\right)_{q}\end{array}\right\}$,

$\mathcal{U}_{3}^{q-R O F}(\mathcal{X})=\left\{\begin{array}{r}\left(u_{k}, \vee_{k=1}^{n}\left(\mu_{1} \widetilde{\mathbb{Q}}_{u_{k}}^{\beta}\left(u_{k}, u_{r}\right) \wedge \mu \mathcal{X}\left(u_{r}\right)\right),\right. \\ \left.\wedge_{k=1}^{n}\left(v_{1} \widetilde{\mathbb{Q}}_{u_{k}}^{\beta}\left(u_{k}, u_{r}\right) \vee v_{\mathcal{X}}\left(u_{r}\right)\right)\right)_{q}\end{array}\right\}$

Then, the pair $\left(\mathcal{L}_{3}^{q-R O F}(\mathcal{X}), \mathcal{U}_{3}^{q-R O F}(\mathcal{X})\right)$ is called the $3-$ $\mathrm{q}-\mathrm{ROF} \beta \mathrm{CRS}$.

Example 14 Consider Example 10 and 11. Then we have the following results.

$$
\begin{gathered}
\mathcal{L}_{3}^{\mathrm{q}-\mathrm{ROF}}(\mathcal{X})=\left\{\begin{array}{r}
\left(u_{1}, 0.35,0.9\right),\left(u_{2}, 0.3,0.95\right), \\
\left(u_{3}, 0.35,0.95\right),\left(u_{4}, 0.55,0.92\right), \\
\left(u_{5}, 0.58,0.9\right),\left(u_{6}, 0.5,0.9\right)
\end{array}\right\}, \\
\mathcal{U}_{3}^{\mathrm{q}-\mathrm{ROF}}(\mathcal{X})=\left\{\begin{array}{c}
\left(u_{1}, 0.98,0.35\right),\left(u_{2}, 0.98,0.35\right), \\
\left(u_{3}, 0.98,0.35\right),\left(u_{4}, 0.98,0.35\right), \\
\left(u_{5}, 0.98,0.35\right),\left(u_{6}, 0.98,0.35\right)
\end{array}\right\} .
\end{gathered}
$$

Theorem 5 Consider a $q$-ROF $\beta C A S(\Theta, \widetilde{\Re})$. Then, we have the following properties

(1) $\mathcal{L}_{3}^{q-R O F}(\mathcal{X})=\left(\mathcal{U}_{3}^{q-R O F}\left(\mathcal{X}^{c}\right)\right)^{c}$.

(2) $\mathcal{U}_{3}^{q-R O F}(\mathcal{X})=\left(\mathcal{L}_{3}^{q-R O F}\left(\mathcal{X}^{c}\right)\right)^{c}$.

(3) If $\mathcal{X} \subseteq \mathcal{Y}$, then $\mathcal{L}_{3}^{q-R O F}(\mathcal{X}) \subseteq \mathcal{L}_{3}^{q-R O F}(\mathcal{Y})$.

(4) If $\mathcal{X} \subseteq \mathcal{Y}$, then $\mathcal{U}_{3}^{q-R O F}(\mathcal{X}) \subseteq \mathcal{U}_{3}^{q-R O F}(\mathcal{Y})$.

(5) $\mathcal{L}_{3}^{q-R O F}(\mathcal{X} \cap \mathcal{Y})=\mathcal{L}_{3}^{q-R O F}(\mathcal{X}) \cap \mathcal{L}_{3}^{q-R O F}(\mathcal{Y})$.

(6) $\mathcal{U}_{3}^{q-R O F}(\mathcal{X} \cap \mathcal{Y}) \subseteq \mathcal{U}_{3}^{q-R O F}(\mathcal{X}) \cap \mathcal{U}_{3}^{q-R O F}(\mathcal{Y})$

(7) $\mathcal{L}_{3}^{q-R O F}(\mathcal{X} \cup \mathcal{Y}) \supseteq \mathcal{L}_{3}^{q-R O F}(\mathcal{X}) \cup \mathcal{L}_{3}^{q-R O F}(\mathcal{Y})$.

(8) $\mathcal{U}_{3}^{q-R O F}(\mathcal{X} \cup \mathcal{Y})=\mathcal{U}_{3}^{q-R O F}(\mathcal{X}) \cup \mathcal{U}_{3}^{q-R O F}(\mathcal{Y})$

(9) $\mathcal{L}_{3}^{q-R O F}(\mathcal{X}) \subseteq \mathcal{X} \subseteq \mathcal{U}_{3}^{q-R O F}(\mathcal{X})$.

Proof It is obvious.

Definition 29 Consider a $\mathrm{q}-\operatorname{ROF} \beta \operatorname{CAS}(\Theta, \widetilde{\Re})$ and $\beta=$ $\left(\mu_{\beta}, v_{\beta}\right)$. Thus the rough and precision degrees of $\mathcal{X} \in$ $\mathrm{q}-\mathrm{ROF}(\Theta)$ are respectively seen as follows.

$$
\begin{aligned}
& { }_{3 \Re^{\Re}} \hat{\mathcal{Q}}^{\beta}(\mathcal{X}) \\
& { }_{3} \mathscr{P}_{\hat{\mathcal{Q}}^{\beta}(\mathcal{X})}=D\left(\mathcal{L}_{3}^{\mathrm{q}-\mathrm{ROF}}(\mathcal{X}), \mathcal{U}_{3}^{\mathrm{q}-\mathrm{ROF}}(\mathcal{X})\right),
\end{aligned}
$$

Example 15 Consider Example 14, then we have the following outcomes.

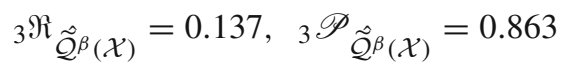

\section{The third type of a Cq-ROFRS}

Definition 30 Consider a $\mathrm{q}-\operatorname{ROF} \beta \operatorname{CAS}(\Theta, \widetilde{\Re})$ and $\beta=$ $\left(\mu_{\beta}, v_{\beta}\right)$. Then for each $u \in \Theta$, define a new kind of q-ROF $\beta$-neighborhood as follows.

${ }_{2} \widetilde{\mathbb{Q}}_{u}^{\beta}=\widetilde{\mathcal{Q}}_{u}^{\beta} \vee \hat{\widetilde{Q}}_{u}^{\beta}$

Example 16 Consider Example 10 and we can obtain the following results for ${ }_{2} \widetilde{\mathbb{Q}}_{u}^{\beta}$ as listed in Table 10 .

Definition 31 Consider a q-ROF $\beta \operatorname{CAS}(\Theta, \widetilde{\Re})$ and $\beta=\left(\mu_{\beta}, v_{\beta}\right)$. For all $u \in \Theta$ and $\mathcal{X} \in q-\operatorname{ROF}(\Theta)$. Define the 4-q-ROFLA $\mathcal{L}_{4}^{q-R O F}(\mathcal{X})$ and 4-q-ROFUA $\mathcal{U}_{4}^{q-R O F}(\mathcal{X})$ as follows.

$$
\begin{gathered}
\mathcal{L}_{4}^{q-R O F}(\mathcal{X})=\left\{\begin{array}{c}
\left(u_{k}, \wedge_{k=1}^{n}\left(\mu_{2} \widetilde{\mathbb{Q}}_{u_{k}}^{\beta}\left(u_{k}, u_{r}\right) \vee \mu \mathcal{X}\left(u_{r}\right)\right),\right. \\
\left.\vee_{k=1}^{n}\left(v_{2} \widetilde{\mathbb{Q}}_{u_{k}}^{\beta}\left(u_{k}, u_{r}\right) \wedge v_{\mathcal{X}}\left(u_{r}\right)\right)\right)_{q}
\end{array}\right\}, \\
\mathcal{U}_{4}^{q-R O F}(\mathcal{X})=\left\{\begin{array}{c}
\left(u_{k}, \vee_{k=1}^{n}\left(\mu_{2} \widetilde{\mathbb{Q}}_{u_{k}}^{\beta}\left(u_{k}, u_{r}\right) \wedge \mu \mathcal{X}\left(u_{r}\right)\right),\right. \\
\left.\wedge_{k=1}^{n}\left(v_{2} \widetilde{\mathbb{Q}}_{u_{k}}^{\beta}\left(u_{k}, u_{r}\right) \vee v_{\mathcal{X}}\left(u_{r}\right)\right)\right)_{q}
\end{array}\right\}
\end{gathered}
$$

Then the pair $\left(\mathcal{L}_{4}^{q-R O F}(\mathcal{X}), \mathcal{U}_{4}^{q-R O F}(\mathcal{X})\right)$ is called the 4-q-ROF $\beta$ CRS.

Example 17 Consider Examples 10 and 11. Then we get the following results.

$$
\begin{gathered}
\mathcal{L}_{4}^{\mathrm{q}-\mathrm{ROF}}(\mathcal{X})=\left\{\begin{array}{c}
\left(u_{1}, 0.58,0.83\right),\left(u_{2}, 0.58,0.83\right), \\
\left(u_{3}, 0.58,0.83\right),\left(u_{4}, 0.58,0.83\right), \\
\left(u_{5}, 0.58,0.83\right),\left(u_{6}, 0.58,0.83\right)
\end{array}\right\}, \\
\mathcal{U}_{4}^{\mathrm{q}-\mathrm{ROF}}(\mathcal{X})=\left\{\begin{array}{c}
\left(u_{1}, 0.98,0.35\right),\left(u_{2}, 0.98,0.3\right), \\
\left(u_{3}, 0.98,0.35\right),\left(u_{4}, 0.98,0.35\right), \\
\left(u_{5}, 0.98,0.35\right),\left(u_{6}, 0.98,0.35\right)
\end{array}\right\} .
\end{gathered}
$$

Theorem 6 Consider a q-ROF $\beta C A S(\Theta, \widetilde{\Re})$. Then, we have the following properties

(1) $\mathcal{L}_{4}^{q-R O F}(\mathcal{X})=\left(\mathcal{U}_{4}^{q-R O F}\left(\mathcal{X}^{c}\right)\right)^{c}$.

(2) $\mathcal{U}_{4}^{q-R O F}(\mathcal{X})=\left(\mathcal{L}_{4}^{q-R O F}\left(\mathcal{X}^{c}\right)\right)^{c}$.

(3) If $\mathcal{X} \subseteq \mathcal{Y}$, then $\mathcal{L}_{4}^{q-R O F}(\mathcal{X}) \subseteq \mathcal{L}_{4}^{q-R O F}(\mathcal{Y})$.

(4) If $\mathcal{X} \subseteq \mathcal{Y}$, then $\mathcal{U}_{4}^{q-R O F}(\mathcal{X}) \subseteq \mathcal{U}_{4}^{q-R O F}(\mathcal{Y})$.

(5) $\mathcal{L}_{4}^{q-R O F}(\mathcal{X} \cap \mathcal{Y})=\mathcal{L}_{4}^{q-R O F}(\mathcal{X}) \cap \mathcal{L}_{4}^{q-R O F}(\mathcal{Y})$ 
Table 9 Result for ${ }_{1} \widetilde{\mathbb{Q}}_{u_{k}}^{\beta}, k=1,2, \ldots, 6$

\begin{tabular}{lllllll}
\hline & $u_{1}$ & $u_{2}$ & $u_{3}$ & $u_{4}$ & $u_{5}$ & $u_{6}$ \\
\hline$\widetilde{\mathbb{Q}}_{u_{1}}^{\beta}$ & $(0.35,0.65)$ & $(0.35,0.65)$ & $(0.7,0.8)$ & $(0.5,0.9)$ & $(0.5,0.9)$ & $(0.7,0.8)$ \\
$1 \widetilde{\mathbb{Q}}_{u_{2}}^{\beta}$ & $(0.65,0.95)$ & $(0.39,0.7)$ & $(0.7,0.79)$ & $(0.65,0.93)$ & $(0.3,0.69)$ & $(0.7,0.79)$ \\
$1 \widetilde{\mathbb{Q}}_{u_{3}}^{\beta}$ & $(0.5,0.9)$ & $(0.35,0.69)$ & $(0.6,0.95)$ & $(0.5,0.9)$ & $(0.69,0.85)$ & $(0.6,0.95)$ \\
$1 \widetilde{\mathbb{Q}}_{u_{4}}^{\beta}$ & $(0.6,0.9)$ & $(0.6,0.9)$ & $(0.55,0.85)$ & $(0.7,0.85)$ & $(0.6,0.92)$ & $(0.55,0.85)$ \\
$1 \widetilde{\mathbb{Q}}_{u_{5}}^{\beta}$ & $(0.6,0.87)$ & $(0.6,0.87)$ & $(0.6,0.87)$ & $(0.6,0.87)$ & $(0.6,0.9)$ & $(0.6,0.8)$ \\
$1 \widetilde{\mathbb{Q}}_{u_{6}}^{\beta}$ & $(0.5,0.9)$ & $(0.6,0.9)$ & $(0.63,0.88)$ & $(0.5,0.89)$ & $(0.6,0.9)$ & $(0.63,0.88)$ \\
\hline
\end{tabular}

Table 10 Result for ${ }_{2} \widetilde{\mathbb{Q}}_{u_{k}}^{\beta}, k=1,2, \ldots, 5$

\begin{tabular}{lllllll}
\hline & $u_{1}$ & $u_{2}$ & $u_{3}$ & $u_{4}$ & $u_{5}$ & $u_{6}$ \\
\hline${ }_{2} \widetilde{\mathbb{Q}}_{u_{1}}^{\beta}$ & $(0.65,0.35)$ & $(0.65,0.35)$ & $(0.8,0.7)$ & $(0.9,0.5)$ & $(0.9,0.5)$ & $(0.8,0.7)$ \\
${ }_{2} \widetilde{\mathbb{Q}}_{u_{2}}^{\beta}$ & $(0.95,0.65)$ & $(0.7,0.39)$ & $(0.79,0.7)$ & $(0.93,0.65)$ & $(0.69,0.3)$ & $(0.79,0.7)$ \\
${ }_{2} \widetilde{\mathbb{Q}}_{u_{3}}^{\beta}$ & $(0.9,0.5)$ & $(0.69,0.35)$ & $(0.95,0.6)$ & $(0.9,0.5)$ & $(0.85,0.69)$ & $(0.95,0.6)$ \\
${ }_{2} \widetilde{\mathbb{Q}}_{u_{4}}^{\beta}$ & $(0.9,0.6)$ & $(0.9,0.6)$ & $(0.85,0.55)$ & $(0.85,0.7)$ & $(0.92,0.6)$ & $(0.85,0.55)$ \\
${ }_{2} \widetilde{\mathbb{Q}}_{u_{5}}^{\beta}$ & $(0.87,0.6)$ & $(0.87,0.6)$ & $(0.87,0.6)$ & $(0.87,0.6)$ & $(0.9,0.6)$ & $(0.8,0.6)$ \\
${ }_{2} \widetilde{\mathbb{Q}}_{u_{6}}^{\beta}$ & $(0.9,0.5)$ & $(0.9,0.6)$ & $(0.88,0.63)$ & $(0.89,0.5)$ & $(0.9,0.6)$ & $(0.88,0.63)$ \\
\hline
\end{tabular}

(6) $\mathcal{U}_{4}^{q-R O F}(\mathcal{X} \cap \mathcal{Y}) \subseteq \mathcal{U}_{4}^{q-R O F}(\mathcal{X}) \cap \mathcal{U}_{4}^{q-R O F}(\mathcal{Y})$.

(7) $\mathcal{L}_{4}^{q-R O F}(\mathcal{X} \cup \mathcal{Y}) \supseteq \mathcal{L}_{4}^{q-R O F}(\mathcal{X}) \cup \mathcal{L}_{4}^{q-R O F}(\mathcal{Y})$.

(8) $\mathcal{U}_{4}^{q-R O F}(\mathcal{X} \cup \mathcal{Y})=\mathcal{U}_{4}^{q-R O F}(\mathcal{X}) \cup \mathcal{U}_{4}^{q-R O F}(\mathcal{Y})$.

(9) $\mathcal{L}_{4}^{q-R O F}(\mathcal{X}) \subseteq \mathcal{X} \subseteq \mathcal{U}_{4}^{q-R O F}(\mathcal{X})$.

\section{Proof}

Definition 32 Consider a $\mathrm{q}-\operatorname{ROF} \beta \operatorname{CAS}(\Theta, \widetilde{\Re})$ and $\beta=$ $\left(\mu_{\beta}, v_{\beta}\right)$. Thus the rough and precision degrees of $\mathcal{X} \in$ $\mathrm{q}-\operatorname{ROF}(\Theta)$ are respectively seen as follows.

$$
\begin{aligned}
& { }_{4} \Re_{\widetilde{\mathcal{Q}}^{\beta}(\mathcal{X})}=D\left(\mathcal{L}_{4}^{\mathrm{q}-\mathrm{ROF}}(\mathcal{X}), \mathcal{U}_{4}^{\mathrm{q}-\mathrm{ROF}}(\mathcal{X})\right), \\
& { }_{4} \mathscr{P}_{\hat{\mathcal{Q}}^{\beta}(\mathcal{X})}=1-{ }_{4} \Re_{\tilde{\mathcal{Q}}^{\beta}(\mathcal{X})} .
\end{aligned}
$$

Example 18 Consider Example 17, then we have the following outcomes.

${ }_{4} \Re_{\hat{\mathcal{Q}}^{\beta}(\mathcal{X})}=0.159,{ }_{4} \mathscr{P}_{\hat{\mathcal{Q}}^{\beta}(\mathcal{X})}=0.841$

\section{Relationships between the proposed methods}

Next, we explain some relationships among these kinds.

Proposition 3 Consider a $q$-ROF $\beta C A S(\Theta, \widetilde{\Re})$ and $\mathcal{X} \in$ $q-R O F(\Theta)$. Then the following properties holds.

(1) $\mathcal{L}_{3}^{q-R O F}(\mathcal{X}) \leq \mathcal{L}_{2}^{q-R O F}(\mathcal{X}) \leq \mathcal{L}_{4}^{q-R O F}(\mathcal{X})$.

(2) $\mathcal{L}_{3}^{q-R O F}(\mathcal{X}) \leq \mathcal{L}_{1}^{q-R O F}(\mathcal{X}) \leq \mathcal{L}_{4}^{q-R O F}(\mathcal{X})$.

(3) $\mathcal{U}_{4}^{q-R O F}(\mathcal{X}) \leq \mathcal{U}_{2}^{q-R O F}(\mathcal{X}) \leq \mathcal{U}_{3}^{q-R O F}(\mathcal{X})$.

(4) $\mathcal{U}_{4}^{q-R O F}(\mathcal{X}) \leq \mathcal{U}_{1}^{q-R O F}(\mathcal{X}) \leq \mathcal{U}_{3}^{q-R O F}(\mathcal{X})$.
Proof The proof is clear from Definitions 28 and 31.

Proposition 4 Consider a q-ROFBCAS $(\Theta, \widetilde{\Re})$ and $\mathcal{X} \in$ $q-\operatorname{ROF}(\Theta)$. Then the following properties holds.

(1) $\mathcal{L}_{4}^{q-R O F}(\mathcal{X}) \geq \mathcal{L}_{1}^{q-R O F}(\mathcal{X}) \cup \mathcal{L}_{2}^{q-R O F}(\mathcal{X})$.
(2) $\mathcal{U}_{4}^{q-R O F}(\mathcal{X}) \leq \mathcal{U}_{1}^{q-R O F}(\mathcal{X}) \cap \mathcal{U}_{2}^{q-R O F}(\mathcal{X})$.
(3) $\mathcal{L}_{3}^{q-R O F}(\mathcal{X}) \leq \mathcal{L}_{1}^{q-R O F}(\mathcal{X}) \cap \mathcal{L}_{2}^{q-R O F}(\mathcal{X})$.
(4) $\mathcal{U}_{3}^{q-R O F}(\mathcal{X}) \geq \mathcal{U}_{1}^{q-R O F}(\mathcal{X}) \cup \mathcal{U}_{2}^{q-R O F}(\mathcal{X})$.

Proof Straightforward.

Remark 2 Considera q-ROF $\beta \operatorname{CAS}(\Theta, \widetilde{\Re})$ and $\mathcal{X} \in \mathrm{q}-\operatorname{ROF}(\Theta)$. From Example 11, you can see the following relations.

(1) $\mathcal{L}_{1}^{\mathrm{q}-\mathrm{ROF}}(\mathcal{X}) \not \leq \mathcal{L}_{2}^{\mathrm{q}-\mathrm{ROF}}(\mathcal{X})$ and $\mathcal{L}_{2}^{\mathrm{q}-\mathrm{ROF}}(\mathcal{X}) \not \leq \mathcal{L}_{1}^{\mathrm{q}-\mathrm{ROF}}(\mathcal{X})$.

(2) $\mathcal{U}_{1}^{\mathrm{q}-\mathrm{ROF}}(\mathcal{X}) \not \mathcal{U}_{2}^{\mathrm{q}-\mathrm{ROF}}(\mathcal{X})$ and $\mathcal{U}_{2}^{\mathrm{q}-\mathrm{ROF}}(\mathcal{X}) \leq \mathcal{U}_{1}^{\mathrm{q}-\mathrm{ROF}}(\mathcal{X})$.

Based on the above remark and Example 11, the two model 1-q-ROF $\beta$ CAS and 2-q-ROF $\beta$ CAS are distinct from some of them.

\section{Decision-making approach using PF $\beta$ CAS}

Now, we illustrate the proposed theoretical study with a real example to clarify how this study is beneficial for the real problems. 


\section{Method on a CPFRS}

\section{Description and process}

Assume that $\Lambda=\left\{u_{r}: r=1, \ldots, k\right\}$ is the set of alternatives, the $m$ main attributes $\tilde{\Upsilon}=\left\{\widetilde{\Delta}_{i}: i=1,2, \ldots, m\right\}$. Then $\tilde{\Upsilon}\left(u_{r}\right)=\left(x_{r j}, y_{r j}\right)$ indicates the experts assessment outcome relevant to the alternatives $u_{r}$ and the attribute $\widetilde{\Delta}_{i}$. Also, we suppose that $\operatorname{PFN} \beta=\left(\vartheta_{\beta}, \zeta_{\beta}\right)$. Thus $(\Lambda, \tilde{\Upsilon})$ is a $\operatorname{PF} \beta C A S$. Therefore, by using the proposed covering method, we set up the following steps to solve problems in MAPFDM.

Step 1: Construct MAPFDM with information system $(\Lambda, \widetilde{\Upsilon}, \mathscr{S}, \mathscr{E})$. So, we have the following formula.

$$
\begin{aligned}
\mathscr{S}_{\uparrow} & =\left\{\widetilde{\Delta}_{j}, \bigvee_{1 \leq i \leq n}\left(\mathcal{R}\left(\tilde{\Delta}_{j}\left(u_{r}\right)\right)\right):(j=1, \ldots, m)\right\} \\
& =\left\{\left(\widetilde{\Delta}_{1}, x_{1}^{\uparrow}, y_{1}^{\uparrow}\right),\left(\tilde{\Delta}_{2}, x_{2}^{\uparrow}, y_{2}^{\uparrow}\right), \ldots,\left(\tilde{\Delta}_{m}, x_{m}^{\uparrow}, y_{m}^{\uparrow}\right)\right\}
\end{aligned}
$$

and

$$
\begin{aligned}
\mathscr{S}_{\downarrow} & =\left\{\widetilde{\Delta}_{j}, \bigwedge_{1 \leq i \leq n}\left(\mathcal{R}\left(\widetilde{\Delta}_{j}\left(u_{r}\right)\right)\right):(j=1, \ldots, m)\right\} \\
& =\left\{\left(\widetilde{\Delta}_{1}, x_{1}^{\downarrow}, y_{1}^{\downarrow}\right),\left(\widetilde{\Delta}_{2}, x_{2}^{\downarrow}, y_{2}^{\downarrow}\right), \ldots,\left(\widetilde{\Delta}_{m}, x_{m}^{\downarrow}, y_{m}^{\downarrow}\right)\right\},
\end{aligned}
$$

where $\bigvee$ and $\bigwedge$ denotes to "max" and "min", respectively, and $\mathcal{R}$ is the score function. If we have $\mathcal{P}=\left(\vartheta_{\mathcal{P}}, \zeta_{\mathcal{P}}\right)$, then $\mathcal{R}(\mathcal{P})=\vartheta_{\mathcal{P}}^{2}-\zeta_{\mathcal{P}}^{2}$ and $-1 \leq \mathcal{R}(\mathcal{P}) \leq 1$

Step 2: Counting the adequate distances $\mathscr{E} \uparrow$ and $\mathscr{E}_{\downarrow}$ as follows:

$$
\begin{aligned}
\mathscr{E} \uparrow= & \sum_{j=1}^{m} \mathcal{T}_{j} \times \mathcal{E}\left[\widetilde{\Delta}_{j}\left(u_{r}\right), \tilde{\Upsilon}_{j}\left(\mathscr{S}_{\uparrow}\right)\right] \\
= & \frac{1}{2} \sum_{j=1}^{m} \mathcal{T}_{j} \times\left[\left|x_{r j}^{2}-x_{j}^{\uparrow^{2}}\right|\right. \\
& \left.+\left|y_{r j}^{2}-y_{j}^{\uparrow}\right|+\left|\xi_{r j}^{2}-\xi_{j}^{\uparrow}\right|\right],
\end{aligned}
$$

and

$$
\begin{aligned}
\mathscr{E}_{\downarrow}= & \sum_{j=1}^{m} \mathcal{T}_{j} \times \mathcal{E}\left[\widetilde{\Delta}_{j}\left(u_{r}\right), \tilde{\Upsilon}_{j}\left(\mathscr{S}_{\downarrow}\right)\right] \\
= & \frac{1}{2} \sum_{j=1}^{m} \mathcal{T}_{j} \times\left[\left|x_{r j}^{2}-x_{j}^{\downarrow^{2}}\right|\right. \\
& \left.+\left|y_{r j}^{2}-y_{j}^{\downarrow^{2}}\right|+\left|\xi_{r j}^{2}-\xi_{j}^{\downarrow^{2}}\right|\right],
\end{aligned}
$$

where $\mathcal{T}_{j}=\left(\mathcal{T}_{1}, \mathcal{T}_{2}, \ldots, \mathcal{T}_{m}\right)$ is the weight vector such that $\sum_{j=1}^{m} \mathcal{T}_{j}=1$. And if we have two PFNs $\mathcal{P}_{1}=\left(\vartheta_{\mathcal{P}_{1}}, \zeta_{\mathcal{P}_{1}}\right)$ and $\mathcal{P}_{2}=\left(\vartheta_{\mathcal{P}_{2}}, \zeta \mathcal{P}_{2}\right)$, then $\mathcal{E}\left(\mathcal{P}_{1}, \mathcal{P}_{2}\right)=\frac{1}{2}\left[\left|\vartheta_{\mathcal{P}_{1}}^{2}-\vartheta_{\mathcal{P}_{2}}^{2}\right|+\right.$
$\left.\left|\zeta_{\mathcal{P}_{1}}^{2}-\zeta_{\mathcal{P}_{2}}^{2}\right|+\left|\xi_{\mathcal{P}_{1}}-\xi_{\mathcal{P}_{2}}\right|\right]$ and $\xi_{\mathcal{P}}=\sqrt{1-\vartheta_{\mathcal{P}}^{2}-\zeta_{\mathcal{P}}^{2}} \cdot \mathscr{E}=$ $\left(\mathscr{E} \uparrow, \mathscr{E}_{\downarrow}\right)$

Step 3: Based on the presented knowledge, calculate the lower and upper approximation of $\mathcal{X}$ using 3-PF $\beta$ CRSs as the following equations.

$\mathcal{L}_{3}^{P F}(\mathscr{E})=\left\{\begin{array}{r}\left(u_{i}, \wedge_{i=1}^{n}\left(\zeta_{\tilde{\mathcal{P}}_{u}^{\beta} \wedge \hat{\mathcal{P}}_{u}^{\beta}}\left(u_{i}, u_{j}\right) \vee \vartheta_{\mathscr{E}}\left(u_{j}\right)\right),\right. \\ \left.\vee_{i=1}^{n}\left(\vartheta_{\widetilde{\mathcal{P}}_{u}^{\beta} \wedge \hat{\mathcal{P}}_{u}^{\beta}}\left(u_{i}, u_{j}\right) \wedge \zeta_{\mathscr{E}}\left(u_{j}\right)\right)\right)\end{array}\right\}$,

$\mathcal{U}_{3}^{P F}(\mathscr{E})=\left\{\begin{aligned}\left(u_{i}, \vee_{i=1}^{n}\left(\zeta_{\widetilde{\mathcal{P}}_{u}^{\beta} \wedge \hat{\mathcal{P}}_{u}^{\beta}}\left(u_{i}, u_{j}\right) \wedge \vartheta_{\mathscr{E}}\left(u_{j}\right)\right),\right. \\ \left.\wedge_{i=1}^{n}\left(\vartheta_{\widetilde{\mathcal{P}}_{u}^{\beta} \wedge \hat{\mathcal{P}}_{u}^{\beta}}\left(u_{i}, u_{j}\right) \vee \zeta_{\mathscr{E}}\left(u_{j}\right)\right)\right)\end{aligned}\right\}$

Step 4: If $0<\gamma \leq 1$ is a threshold and $\mathscr{T}(u, v)=$ $\frac{u v}{\sqrt{1+\left(1-u^{2}\right)\left(1-v^{2}\right)}}$. Calculate the sorting function of the MAPFDM problem as follows.

$\varkappa\left(u_{i}\right)=\gamma \times \mathscr{T}\left(\mathcal{L}_{3}^{P F}(\mathscr{E})\right)+(1-\gamma) \times \mathscr{T}\left(\mathcal{U}_{3}^{P F}(\mathscr{E})\right)$

and hence sorting the alternatives.

The following algorithm is established from the above data and it put forward in Algorithm 1.

Algorithm 1. Algorithm for a PF $\beta$ CRSs to make a decision.

Input: MAPFDM with information system $(\Lambda, \tilde{\Upsilon}, \mathscr{S}, \mathscr{E})$.

Output: Decision Making.

1: Compute $\mathscr{S}_{\uparrow}$ and $\mathscr{S}_{\downarrow}$.

2: Compute $\mathscr{E} \uparrow$ and $\mathscr{E}_{\downarrow}$ 3: From Definition 6, compute $\widetilde{\mathcal{P}}_{u}^{\beta}$.

4: From Step 2 and by Definition 15, compute $\hat{\widetilde{\mathcal{P}}}_{u}^{\beta}$.

5: From Steps 2 and 3, compute 3-PF $\beta$ CRSs $\mathcal{L}_{3}^{P F}(\mathscr{E})$ and $\mathcal{U}_{3}^{P F}(\mathscr{E})$

6: Compute the sorting function $\varkappa\left(u_{i}\right)$.

7: Obtain the decision.

\section{Test example}

Presume that $\Lambda=\left\{u_{1}, u_{2}, \ldots, u_{8}\right\}$ is a set of eight emergency plans and $\widetilde{\Upsilon}=\left\{\right.$ Comprehensiveness and completeness $\left(\widetilde{\Delta}_{1}\right)$, Timely Response $\left(\widetilde{\Delta}_{2}\right)$, Feasibility $\left(\widetilde{\Delta}_{3}\right)$, Budgeting the cost $\left(\widetilde{\Delta}_{4}\right)$, Ability to adjust the plan $\left.\left(\widetilde{\Delta}_{5}\right)\right\}$ is the attribute set. The proceedings of the mentioned algorithm 1 are indicated below.

Step 1: In the set of attributes, an expert analyses each alternative and provides its conclusions with relevant values that are concise in Table 11.

Step 2: Expert gives the following results, according to the significance of these five attributes. 
Table 11 Result for $(\Lambda, \tilde{\Upsilon})$

\begin{tabular}{llllll}
\hline & $\widetilde{\Delta}_{1}$ & $\widetilde{\Delta}_{2}$ & $\widetilde{\Delta}_{3}$ & $\widetilde{\Delta}_{4}$ & $\tilde{\Delta}_{5}$ \\
\hline$u_{1}$ & $(0.7,0.1)$ & $(0.8,0.5)$ & $(0.8,0.1)$ & $(0.9,0.4)$ & $(0.6,0.5)$ \\
$u_{2}$ & $(0.4,0.3)$ & $(0.7,0.2)$ & $(0.3,0.9)$ & $(0.6,0.3)$ & $(0.3,0.8)$ \\
$u_{3}$ & $(0.2,0.6)$ & $(0.7,0.4)$ & $(0.7,0.7)$ & $(0.3,0.9)$ & $(0.7,0.1)$ \\
$u_{4}$ & $(0.7,0.5)$ & $(0.4,0.8)$ & $(0.6,0.1)$ & $(0.5,0.4)$ & $(0.7,0.4)$ \\
$u_{5}$ & $(0.6,0.6)$ & $(0.9,0.3)$ & $(0.7,0.1)$ & $(0.6,0.3)$ & $(0.7,0.7)$ \\
$u_{6}$ & $(0.5,0.3)$ & $(0.5,0.7)$ & $(0.5,0.2)$ & $(0.9,0.4)$ & $(0.7,0.3)$ \\
$u_{7}$ & $(0.6,0.5)$ & $(0.6,0.1)$ & $(0.4,0.6)$ & $(0.8,0.5)$ & $(0.6,0.2)$ \\
$u_{8}$ & $(0.8,0.3)$ & $(0.7,0.7)$ & $(0.2,0.6)$ & $(0.6,0.3)$ & $(0.4,0.5)$ \\
\hline
\end{tabular}

$$
\begin{aligned}
\mathscr{S}_{\uparrow}= & \left\{\left(\widetilde{\Delta}_{1}, 0.8,0.3\right),\left(\widetilde{\Delta}_{2}, 0.9,0.3\right),\left(\widetilde{\Delta}_{3}, 0.8,0.1\right),\right. \\
& \left.\left(\widetilde{\Delta}_{4}, 0.9,0.4\right),\left(\widetilde{\Delta}_{5}, 0.7,0.1\right)\right\} . \\
\mathscr{S}_{\downarrow}= & \left\{\left(\widetilde{\Delta}_{1}, 0.2,0.6\right),\left(\widetilde{\Delta}_{2}, 0.4,0.8\right),\left(\widetilde{\Delta}_{3}, 0.3,0.9\right),\right. \\
& \left.\left(\widetilde{\Delta}_{4}, 0.3,0.9\right),\left(\widetilde{\Delta}_{5}, 0.3,0.8\right)\right\} .
\end{aligned}
$$

Step 3: If we have the following weights $\mathcal{T}_{1}=0.18, \mathcal{T}_{2}=$ $0.32, \mathcal{T}_{3}=0.1, \mathcal{T}_{4}=0.25, \mathcal{T}_{5}=0.15$, then we compute the suitable distances as follows.

$$
\begin{array}{r}
\mathscr{E}_{\uparrow}=\left\{\begin{array}{r}
\left(u_{1}, 0.1249\right),\left(u_{2}, 0.4466\right),\left(u_{3}, 0.3945\right), \\
\left(u_{4}, 0.3871\right),\left(u_{5}, 0.2213\right),\left(u_{6}, 0.2822\right), \\
\left(u_{7}, 0.2945\right),\left(u_{8}, 0.3289\right)
\end{array}\right\} \\
\mathscr{E}_{\downarrow}=\left\{\begin{array}{r}
\left(u_{1}, 0.5265\right),\left(u_{2}, 0.3914\right),\left(u_{3}, 0.2698\right), \\
\left(u_{4}, 0.3628\right),\left(u_{5}, 0.5299\right),\left(u_{6}, 0.4154\right), \\
\left(u_{7}, 0.5101\right),\left(u_{8}, 0.4452\right)
\end{array}\right\}
\end{array}
$$

Therefore,

$$
\mathscr{E}=\left\{\begin{array}{c}
\left(u_{1}, 0.1249,0.5265\right),\left(u_{2}, 0.4466,0.3914\right), \\
\left(u_{3}, 0.3945,0.2698\right),\left(u_{4}, 0.3871,0.3628\right), \\
\left(u_{5}, 0.2213,0.5299\right),\left(u_{6}, 0.2822,0.4154\right), \\
\left(u_{7}, 0.2945,0.5101\right),\left(u_{8}, 0.3289,0.4452\right)
\end{array}\right\}
$$

Step 4: Using 3-PF $\beta$ CRSs, compute the lower and upper approximation as the following results.
First, we calculate the PF $\beta$-neighborhoods as follows.

$$
\begin{aligned}
& \widetilde{\mathcal{P}}_{u_{1}}^{(0.7,0.4)}=\widetilde{\Delta}_{1} \cap \widetilde{\Delta}_{2} \cap \widetilde{\Delta}_{3} \cap \widetilde{\Delta}_{4}, \\
& \widetilde{\mathcal{P}}_{u_{2}}^{(0.7,0.4)}=\widetilde{\Delta}_{2}, \\
& \widetilde{\mathcal{P}}_{u_{3}}^{(0.7,0.4)}=\widetilde{\Delta}_{2} \cap \widetilde{\Delta}_{5}, \\
& \widetilde{\mathcal{P}}_{u_{4}}^{(0.7,0.4)}=\widetilde{\Delta}_{3} \cap \widetilde{\Delta}_{5}, \\
& \widetilde{\mathcal{P}}_{u_{5}}^{(0.7,0.4)}=\widetilde{\Delta}_{2} \cap \widetilde{\Delta}_{3}, \\
& \widetilde{\mathcal{P}}_{u_{6}}^{(0.7,0.4)}=\widetilde{\Delta}_{4} \cap \widetilde{\Delta}_{5}, \\
& \widetilde{\mathcal{P}}_{u_{7}}^{(0.7,0.4)}=\widetilde{\Delta}_{2} \cap \widetilde{\Delta}_{4}, \\
& \widetilde{\mathcal{P}}_{u_{8}}^{(0.7,0.4)}=\widetilde{\Delta}_{1}
\end{aligned}
$$

The complete values of $\widetilde{\mathcal{P}}_{u_{s}}^{(0.7,0.4)}, s=1,2, \ldots, 6$ are given in Table 12.

The PF complementary $\beta$-neighborhood of $\widetilde{\mathcal{P}}_{u_{s}}^{(0.7,0.4)}, s=$ $1,2, \ldots, 6$ is given in Table 13 .

Moreover, the $\widetilde{\mathbb{T}}_{u}^{\beta}$ neighborhood as follow in Table 14.

Then using such information, we can obtain the results as follows.

$$
\begin{gathered}
\mathcal{L}_{3}^{\mathrm{PF}}(\mathscr{E})=\left\{\begin{array}{r}
\left(u_{1}, 0.6,0.5299\right),\left(u_{2}, 0.6,0.5\right), \\
\left(u_{3}, 0.6,0.5299\right),\left(u_{4}, 0.5,0.5299\right), \\
\left(u_{5}, 0.6,0.5\right),\left(u_{6}, 0.5,0.5299\right), \\
\left(u_{7}, 0.6,0.5\right),\left(u_{8}, 0.4466,0.5299\right)
\end{array}\right\}, \\
\mathcal{U}_{3}^{\mathrm{PF}}(\mathscr{E})=\left\{\begin{array}{r}
\left(u_{1}, 0.3945,0.6\right),\left(u_{2}, 0.3945,0.6\right), \\
\left(u_{3}, 0.3871,0.6\right),\left(u_{4}, 0.3945,0.5\right), \\
\left(u_{5}, 0.3945,0.6\right),\left(u_{6}, 0.3871,0.5\right), \\
\left(u_{7}, 0.3871,0.6\right),\left(u_{8}, 0.3871,0.4\right)
\end{array}\right\}
\end{gathered}
$$

Step 5: Compute the sorting function $\varkappa(u)$ as follows.

$$
\varkappa\left(u_{i}\right)=\left\{\begin{array}{r}
\left(u_{1}, 0.2486\right),\left(u_{2}, 0.2354\right), \\
\left(u_{3}, 0.2479\right),\left(u_{4}, 0.202\right), \\
\left(u_{5}, 0.2354\right),\left(u_{6}, 0.209\right), \\
\left(u_{7}, 0.2347\right),\left(u_{8}, 0.1745\right)
\end{array}\right\},
$$

Table 12 Result for $\widetilde{\mathcal{P}}_{u_{s}}^{(0.7,0.4)}, s=1,2, \ldots, 6$

\begin{tabular}{lllllllll}
\hline & $u_{1}$ & $u_{2}$ & $u_{3}$ & $u_{4}$ & $u_{5}$ & $u_{6}$ & $u_{7}$ & $u_{8}$ \\
\hline$\widetilde{\mathcal{P}}_{u_{1}}^{\beta}$ & $(0.7,0.5)$ & $(0.3,0.9)$ & $(0.2,0.9)$ & $(0.4,0.8)$ & $(0.6,0.6)$ & $(0.5,0.7)$ & $(0.4,0.6)$ & $(0.2,0.7)$ \\
$\widetilde{\mathcal{P}}_{u_{2}}^{\beta}$ & $(0.8,0.5)$ & $(0.7,0.2)$ & $(0.7,0.4)$ & $(0.4,0.8)$ & $(0.9,0.3)$ & $(0.5,0.7)$ & $(0.6,0.1)$ & $(0.7,0.7)$ \\
$\widetilde{\mathcal{P}}_{u_{3}}^{\beta}$ & $(0.6,0.5)$ & $(0.3,0.8)$ & $(0.7,0.4)$ & $(0.4,0.8)$ & $(0.7,0.7)$ & $(0.5,0.7)$ & $(0.6,0.2)$ & $(0.4,0.7)$ \\
$\widetilde{\mathcal{P}}_{u_{4}}^{\beta}$ & $(0.6,0.5)$ & $(0.3,0.9)$ & $(0.7,0.7)$ & $(0.6,0.4)$ & $(0.7,0.7)$ & $(0.5,0.3)$ & $(0.4,0.6)$ & $(0.2,0.6)$ \\
$\widetilde{\mathcal{P}}_{u_{5}}^{\beta}$ & $(0.8,0.5)$ & $(0.3,0.9)$ & $(0.7,0.7)$ & $(0.4,0.8)$ & $(0.7,0.3)$ & $(0.5,0.7)$ & $(0.4,0.6)$ & $(0.2,0.7)$ \\
$\widetilde{\mathcal{P}}_{u_{6}}^{\beta}$ & $(0.6,0.5)$ & $(0.3,0.8)$ & $(0.3,0.9)$ & $(0.5,0.4)$ & $(0.6,0.7)$ & $(0.7,0.4)$ & $(0.6,0.5)$ & $(0.4,0.5)$ \\
$\widetilde{\mathcal{P}}_{u_{7}}^{\beta}$ & $(0.8,0.5)$ & $(0.6,0.3)$ & $(0.3,0.9)$ & $(0.4,0.8)$ & $(0.6,0.3)$ & $(0.5,0.7)$ & $(0.6,0.5)$ & $(0.6,0.7)$ \\
$\widetilde{\mathcal{P}}_{u_{8}}^{\beta}$ & $(0.7,0.1)$ & $(0.4,0.3)$ & $(0.2,0.6)$ & $(0.7,0.5)$ & $(0.6,0.6)$ & $(0.5,0.3)$ & $(0.6,0.5)$ & $(0.8,0.3)$ \\
\hline & & & & & & & &
\end{tabular}


Table 13 Result for $\widetilde{\mathcal{P}}_{u_{s}}^{(0 . \hat{7}, 0.4)}, s=1,2, \ldots, 6$

\begin{tabular}{|c|c|c|c|c|c|c|c|c|}
\hline & $u_{1}$ & $u_{2}$ & $u_{3}$ & $u_{4}$ & $u_{5}$ & $u_{6}$ & $u_{7}$ & $u_{8}$ \\
\hline$\hat{\widetilde{\mathcal{P}}_{u_{1}}^{\beta}}$ & $(0.5,0.7)$ & $(0.9,0.3)$ & $(0.9,0.2)$ & $(0.8,0.4)$ & $(0.6,0.6)$ & $(0.7,0.5)$ & $(0.6,0.4)$ & $(0.7,0.2$ \\
\hline$\hat{\widetilde{\mathcal{P}}}_{u_{2}}^{\beta}$ & $(0.5,0.8)$ & $(0.2,0.7)$ & $(0.4,0.7)$ & $(0.8,0.4)$ & $(0.3,0.9)$ & $(0.7,0.5)$ & $(0.1,0.6)$ & $(0.7,0.7)$ \\
\hline$\hat{\widetilde{\mathcal{P}}}_{u_{3}}^{\beta}$ & $(0.5,0.6)$ & $(0.8,0.3)$ & $(0.4$, & $(0.8$ & $(0.7$, & $(0.7,0.5)$ & $(0.2,0.6)$ & $0.7,0.4$ \\
\hline$\hat{\widetilde{\mathcal{P}}}_{u_{4}}^{\beta}$ & $(0.5,0.6)$ & $(0.9,0.3)$ & $(0.7,0.7)$ & $(0.4,0.6)$ & $(0.7,0.7)$ & $(0.3,0.5)$ & $(0.6,0.4)$ & $(0.6,0.2)$ \\
\hline$\hat{\widetilde{\mathcal{P}}}_{u_{5}}^{\beta}$ & $(0.5,0.8)$ & $(0.9,0.3)$ & $(0.7,0.7)$ & $(0.8,0.4)$ & $(0.3,0.7)$ & $(0.7,0.5)$ & $(0.6,0.4)$ & $(0.7,0.2)$ \\
\hline$\hat{\widetilde{\mathcal{P}}}_{u_{6}}^{\beta}$ & $(0.5,0.6)$ & $(0.8,0.3)$ & $(0.9,0.3)$ & $(0.4,0.5)$ & $(0.7,0.6)$ & $(0.4,0.7)$ & $(0.5,0.6)$ & $(0.5,0.4)$ \\
\hline$\hat{\widetilde{\mathcal{P}}}_{u_{7}}^{\beta}$ & $(0.5,0.8)$ & $(0.3,0.6)$ & $(0.9,0.3)$ & $(0.8,0.4)$ & $(0.3,0.6)$ & $(0.7,0.5)$ & $(0.5,0.6)$ & $(0.7,0.6)$ \\
\hline$\hat{\widetilde{\mathcal{P}}}_{u_{8}}^{\beta}$ & $(0.1,0.7)$ & $(0.3,0.4)$ & $(0.6,0.2)$ & $(0.5,0.7)$ & $(0.6,0.6)$ & $(0.3,0.5)$ & $(0.5,0.6)$ & $(0.3,0.8)$ \\
\hline
\end{tabular}

Table 14 Result for $\widetilde{\mathcal{P}}_{u_{s}}^{(0.7,0.4)}, s=1,2, \ldots, 6$

\begin{tabular}{|c|c|c|c|c|c|c|c|c|}
\hline & $u_{1}$ & $u_{2}$ & $u_{3}$ & $u_{4}$ & $u_{5}$ & $u_{6}$ & $u_{7}$ & $u_{8}$ \\
\hline${ }_{1} \widetilde{\mathbb{I}}_{u_{1}}^{\beta}$ & $(0.5,0.7)$ & $(0.3,0.9)$ & $(0.2,0.9)$ & $(0.4,0.8)$ & $(0.6,0.6)$ & $(0.5,0.7)$ & $(0.4,0.6)$ & $(0.2,0.7)$ \\
\hline$\widetilde{\mathbb{1}}_{u_{2}}^{\beta}$ & $(0.5,0.8)$ & $(0.2,0.7)$ & $(0.4,0.7)$ & $(0.4,0.8)$ & $(0.3,0.9)$ & $(0.5,0.7)$ & $(0.1,0.6)$ & $(0.7,0.7)$ \\
\hline${ }_{1} \widetilde{\mathbb{U}}_{u_{3}}^{\beta}$ & $(0.5,0.6)$ & $(0.3,0.8)$ & $(0.4,0.7)$ & $(0.4,0.8)$ & $(0.7,0.7)$ & $(0.5,0.7)$ & $(0.2,0.6)$ & $(0.4,0.7)$ \\
\hline${ }_{1} \widetilde{\mathbb{U}}_{u_{4}}^{\beta}$ & $(0.5,0.6)$ & $(0.3,0.9)$ & $(0.7,0.7)$ & $(0.4,0.6)$ & $(0.7,0.7)$ & $(0.3,0.5)$ & $(0.4,0.6)$ & $(0.2,0.6)$ \\
\hline${ }_{1} \widetilde{\mathbb{I}}_{u_{5}}^{\beta}$ & $(0.5,0.8)$ & $(0.3,0.9)$ & $(0.7,0.7)$ & $(0.4,0.8)$ & $(0.3,0.7)$ & $(0.5,0.7)$ & $(0.4,0.6)$ & $(0.2,0.7)$ \\
\hline${ }_{1} \widetilde{\mathbb{I}}_{u_{6}}^{\beta}$ & $(0.5,0.6)$ & $(0.3,0.8)$ & $(0.3,0.9)$ & $(0.4,0.5)$ & $(0.6,0.7)$ & $(0.4,0.7)$ & $(0.5,0.6)$ & $(0.4,0.5)$ \\
\hline${ }_{1} \widetilde{\mathbb{d}}_{u_{7}}^{\beta}$ & $(0.5,0.8)$ & $(0.3,0.6)$ & $(0.3,0.9)$ & $(0.4,0.8)$ & $(0.3,0.6)$ & $(0.5,0.7)$ & $(0.5,0.6)$ & $(0.6,0.7)$ \\
\hline${ }_{1} \widetilde{\Psi}_{u_{8}}^{\beta}$ & $(0.1,0.7)$ & $(0.3,0.4)$ & $(0.2,0.6)$ & $(0.5,0.7)$ & $(0.6,0.6)$ & $(0.3,0.5)$ & $(0.5,0.6)$ & $(0.3,0.8)$ \\
\hline
\end{tabular}

and the sorting values as below

$u_{1} \geq u_{3} \geq u_{2} \approx u_{5} \geq u_{7} \geq u_{6} \geq u_{4} \geq u_{8}$.

\section{Method on a Cq-ROFRS}

\section{Description and process}

Assume that $\Theta=\left\{u_{r}: r=1, \ldots, k\right\}$ is the set of alternatives, the $m$ main attributes $\widetilde{\Re}=\left\{\widetilde{\delta}_{i}: i=1,2, . ., m\right\}$. Then $\hat{\mathcal{E}}_{\text {in }}$ and $\hat{\mathcal{E}}_{\text {out }}$ are the experts assessment outcomes relevant to the alternatives $u_{r}$ and the attribute $\tilde{\delta}_{i}$ by $\mu_{r i}$ and $v_{r i}$. Thus $\tilde{\delta}_{i}\left(u_{r}\right)=\left(\mu_{r i}, v_{r i}\right)$ is q-ROFN and represents by the following matrix, where $\hat{\mathcal{E}}_{\text {in }}$ is the membership grade $\mu_{r i}$ and $\hat{\mathcal{E}}_{\text {out }}$ is the membership grade $v_{r i}$.

$\tilde{\delta}_{i}\left(u_{r}\right)=\left(\begin{array}{cccc}\left(\mu_{11}, v_{11}\right) & \left(\mu_{12}, v_{12}\right) & \cdots & \left(\mu_{1 i}, v_{1 i}\right) \\ \left(\mu_{21}, v_{21}\right) & \left(\mu_{22}, v_{22}\right) & \cdots & \left(\mu_{2 i}, v_{2 i}\right) \\ \vdots & \vdots & \ddots & \vdots \\ \left(\mu_{r 1}, v_{r 1}\right) & \left(\mu_{r 2}, v_{r 2}\right) & \cdots & \left(\mu_{r i}, v_{r i}\right)\end{array}\right)$

So, $(\Theta, \widetilde{\Re})$ is a q-ROF $\beta$ CAS. Therefore, we give the following steps to solve MADM problems through presented method on a Cq-ROFRS.
Step 1: Construct q-ROF plus ideal and q-ROF minus ideal as the following formulas.

$$
\begin{aligned}
\mathscr{L}^{\oplus} & =\left\{\widetilde{\delta}_{j}, \bigvee_{1 \leq i \leq n}\left(\mathcal{S}\left(\widetilde{\delta}_{j}\left(u_{r}\right)\right)\right):(j=1, \ldots, m)\right\} \\
& =\left\{\left(\tilde{\delta}_{1}, x_{1}^{\oplus}, y_{1}^{\oplus}\right),\left(\tilde{\delta}_{2}, x_{2}^{\oplus}, y_{2}^{\oplus}\right), \ldots,\left(\widetilde{\delta}_{m}, x_{m}^{\oplus}, y_{m}^{\oplus}\right)\right\}
\end{aligned}
$$

and

$$
\begin{aligned}
\mathscr{L}^{\ominus} & =\left\{\tilde{\delta}_{j}, \bigwedge_{1 \leq i \leq n}\left(\mathcal{S}\left(\tilde{\delta}_{j}\left(u_{r}\right)\right)\right):(j=1, \ldots, m)\right\} \\
& =\left\{\left(\tilde{\delta}_{1}, x_{1}^{\ominus}, y_{1}^{\ominus}\right),\left(\tilde{\delta}_{2}, x_{2}^{\ominus}, y_{2}^{\ominus}\right), \ldots,\left(\tilde{\delta}_{m}, x_{m}^{\ominus}, y_{m}^{\ominus}\right)\right\},
\end{aligned}
$$

where $\bigvee$ and $\bigwedge$ denotes to "max" and "min", respectively, and $\mathcal{S}$ is the score function. If we have $\mathcal{X}=\left(\mu_{\mathcal{X}}, v_{\mathcal{X}}\right)$, then $\mathcal{S}(\mathcal{X})=\frac{1}{2}\left(1+\mu_{\mathcal{X}}^{q}-v_{\mathcal{X}}^{q}\right)$ and $q \geq 1$.

Step 2: Counting the adequate distances $\mathscr{D}^{\oplus}$ and $\mathscr{D}^{\ominus}$ as follows: 


$$
\begin{aligned}
\mathscr{D}^{\oplus}= & \sum_{j=1}^{m} \mathcal{W}_{j} D\left(\widetilde{\delta}_{j}\left(u_{r}\right), \tilde{\delta}_{j}\left(\mathscr{L}^{\oplus}\right)\right) \\
= & \left(\frac{1}{2 n} \sum_{j=1}^{m} \mathcal{W}_{j}\left|\mu_{r j}-\mu_{j}^{\oplus}\right|^{q}\right. \\
& \left.+\frac{1}{2 n} \sum_{j=1}^{m} \mathcal{W}_{j}\left|v_{r j}-v_{j}^{\oplus}\right|^{q}\right)^{\frac{1}{q}}, \\
\mathscr{D}^{\ominus}= & \sum_{j=1}^{m} \mathcal{W}_{j} D\left(\tilde{\delta}_{j}\left(u_{r}\right), \tilde{\delta}_{j}\left(\mathscr{L}^{\ominus}\right)\right) \\
= & \left(\frac{1}{2 n} \sum_{j=1}^{m} \mathcal{W}_{j}\left|\mu_{r j}-\mu_{j}^{\ominus}\right|^{q}\right. \\
& \left.+\frac{1}{2 n} \sum_{j=1}^{m} \mathcal{W}_{j}\left|v_{r j}-v_{j}^{\ominus}\right|^{q}\right)^{\frac{1}{q}},
\end{aligned}
$$

where $\mathcal{W}_{j}=\left(\mathcal{W}_{1}, \mathcal{W}_{2}, \ldots, \mathcal{W}_{m}\right)$ is the weight vector such that $\sum_{j=1}^{m} \mathcal{W}_{j}=1$. So, we have $\mathscr{D}=\left(\mathscr{D}^{\oplus}, \mathscr{D}^{\ominus}\right)$.

Step 3: Compute the lower and upper approximation of $\mathcal{X}$ using 4-q-ROF $\beta$ CRSs as the following equations.

$$
\mathcal{L}_{4}^{\mathrm{q}-\mathrm{ROF}}(\mathscr{D})=\left\{\begin{array}{c}
\left(u_{i}, \wedge_{j=1}^{m}\left(\mu_{2} \widetilde{\mathbb{Q}}_{u_{i}}^{\beta}\left(u_{i}, u_{j}\right) \wedge \mu_{\mathscr{D}}\left(u_{j}\right)\right)\right), \\
\left(u_{i}, \vee_{j=1}^{m}\left(v_{2} \widetilde{\mathbb{Q}}_{u_{i}}^{\beta}\left(u_{i}, u_{j}\right) \vee v_{\mathscr{D}}\left(u_{j}\right)\right)\right)
\end{array}\right\},
$$

$\mathcal{U}_{4}^{\mathrm{q}-\mathrm{ROF}}(\mathscr{D})=\left\{\begin{array}{c}\left(u_{i}, \vee_{j=1}^{m}\left(\mu_{2} \widetilde{\mathbb{Q}}_{u_{i}}^{\beta}\left(u_{i}, u_{j}\right) \vee \mu_{\mathscr{D}}\left(u_{j}\right)\right)\right), \\ \left(u_{i}, \wedge_{j=1}^{m}\left(v_{2} \widetilde{\mathbb{Q}}_{u_{i}}^{\beta}\left(u_{i}, u_{j}\right) \wedge v_{\mathscr{D}}\left(u_{j}\right)\right)\right)\end{array}\right\}$

Step 4: If $0<\xi \leq 1$ is a threshold and $\mathcal{T}(u, v)=$ $\frac{u v}{\sqrt[q]{1+\left(1-u^{q}\right)\left(1-v^{q}\right)}}$, then compute the sorting function of the MAq-ROFDM problem as follows.

$$
\varphi\left(u_{i}\right)=\xi \times \mathcal{T}\left(\mathcal{L}_{4}^{\mathrm{q}-\mathrm{ROF}}(\mathscr{D})\right)+(1-\xi) \times \mathcal{T}\left(\mathcal{U}_{4}^{\mathrm{q}-\mathrm{ROF}}(\mathscr{D})\right)
$$

and hence sorting the alternatives.

The following algorithm is established from the above data and it put forward in Algorithm 2.
Algorithm 2. Algorithm for a q-ROF $\beta$ CRSs to make a decision.

Input: MAq-ROFDM with information system $(\Theta, \widetilde{\Re}, \mathscr{L}, \mathscr{D})$.

Output: Decision Making.

1: Compute $\mathscr{L}^{\oplus}$ and $\mathscr{L}^{\ominus}$.

2: Compute $\mathscr{D}^{\oplus}$ and $\mathscr{D}^{\ominus}$ 3: From Definition 12, compute $\widetilde{\mathcal{Q}}_{u}^{\beta}$.

4: From Step 2 and by Definition 24, compute $\hat{\widetilde{\mathcal{Q}}}_{u}^{\beta}$.

5: From Steps 2 and 3, compute 4 -q-ROF $\beta \operatorname{CRSs} \mathcal{L}_{4}^{q-R O F}(\mathscr{D})$ and $\mathcal{U}_{4}^{q-R O F}(\mathscr{D})$

6: Compute the sorting function $\varphi\left(u_{i}\right)$.

7: Obtain the decision.

\section{Test example}

Assume that $\Theta=\left\{u_{1}, u_{2}, \ldots, u_{5}\right\}$ is a set of five candidates for the faculty position in $U$ university and $\widetilde{\Re}=\{$ Research productivity $\left(\widetilde{\delta}_{1}\right)$, Managerial skill $\left(\widetilde{\delta}_{2}\right)$, Impact on research community $\left(\widetilde{\delta}_{3}\right)$, Ability to work under pressure $\left(\widetilde{\delta}_{4}\right)$, Academic leadership qualities $\left(\widetilde{\delta}_{5}\right)$, Contribution to $U$ University $\left.\left(\tilde{\delta}_{6}\right)\right\}$ is the attribute set. The proceedings of the mentioned algorithm 2 are indicated below.

Step 1: The decision maker's evaluate $\hat{\mathcal{E}}_{\text {in }}$ and $\hat{\mathcal{E}}_{\text {out }}$ are established in Table 15.

\begin{tabular}{|c|c|c|c|c|c|c|}
\hline & $\tilde{\delta}_{1}$ & $\widetilde{\delta}_{2}$ & $\widetilde{\delta}_{3}$ & $\tilde{\delta}_{4}$ & $\tilde{\delta}_{5}$ & $\tilde{\delta}_{6}$ \\
\hline$u_{1}$ & $(0.93,0.3)$ & $(0.7,0.4)$ & $(0.8,0.2)$ & $(0.9,0.1)$ & $(0.7,0.6)$ & $(0.4,0.3)$ \\
\hline$u_{2}$ & $(0.9,0.4)$ & $(0.7,0.8)$ & $(0.7,0.8)$ & $(0.6,0.3)$ & $(0.65,0.87)$ & $(0.6,0.2)$ \\
\hline$u_{3}$ & $(0.8,0.7)$ & $(0.7,0.2)$ & $(0.95,0.4)$ & $(0.8,0.4)$ & $(0.5,0.2)$ & $(0.8,0.3)$ \\
\hline$u_{4}$ & $(0.8,0.3)$ & $(0.6,0.3)$ & $(0.7,0.4)$ & $(0.9,0.2)$ & $(0.8,0.65)$ & $(0.4,0.2)$ \\
\hline$u_{5}$ & $(0.5,0.2)$ & $(0.95,0.4)$ & $(0.8,0.3)$ & $(0.7,0.1)$ & $(0.6,0.3)$ & $(0.94,0.33)$ \\
\hline
\end{tabular}

Step 2: Compute $\mathscr{L}^{\oplus}$ and $\mathscr{L}^{\ominus}$ as the following.

$$
\begin{aligned}
& \mathscr{L}^{\oplus}=\left\{\begin{array}{c}
\left(\tilde{\delta}_{1}, 0.98,0.3\right),\left(\tilde{\delta}_{2}, 0.95,0.4\right),\left(\tilde{\delta}_{3}, 0.95,0.4\right), \\
\left(\tilde{\delta}_{4}, 0.9,0.1\right),\left(\tilde{\delta}_{5}, 0.8,0.65\right),\left(\tilde{\delta}_{6}, 0.94,0.38\right)
\end{array}\right\}, \\
& \mathscr{L}^{\ominus}=\left\{\begin{array}{c}
\left(\tilde{\delta}_{1}, 0.5,0.2\right),\left(\tilde{\delta}_{2}, 0.7,0.8\right),\left(\tilde{\delta}_{3}, 0.7,0.5\right), \\
\left(\tilde{\delta}_{4}, 0.6,0.3\right),\left(\tilde{\delta}_{5}, 0.65,0.87\right),\left(\tilde{\delta}_{6}, 0.4,0.3\right)
\end{array}\right\}
\end{aligned}
$$

Step 3: If we have the weights for all attribute as follows $\mathcal{W}_{j}=(0.2,0.18,0.22,0.12,0.15,0.13)$, then we compute the distances as indicated below.

$$
\begin{aligned}
\mathscr{D}^{\oplus}= & \left\{\left(u_{1}, 0.12943\right),\left(u_{2}, 0.13642\right),\right. \\
& \left.\left(u_{3}, 0.14903\right),\left(u_{4}, 0.14129\right),\left(u_{5}, 0.13849\right)\right\}, \\
\mathscr{D}^{\ominus}= & \left\{\left(u_{1}, 0.15782\right),\left(u_{2}, 0.10907\right),\right. \\
& \left.\left(u_{3}, 0.2201\right),\left(u_{4}, 0.11109\right),\left(u_{5}, 0.17627\right)\right\} .
\end{aligned}
$$

Table 15 Result for $(\Theta, \widetilde{\Re})$ 
Table 16 Result for $\widetilde{\mathcal{Q}}_{u_{k}}^{(0.8,0.4)}, k=1,2, \ldots, 5$

\begin{tabular}{llllll}
\hline & $u_{1}$ & $u_{2}$ & $u_{3}$ & $u_{4}$ & $u_{5}$ \\
\hline$\widetilde{\mathcal{Q}}_{u_{1}}^{\beta}$ & $(0.8,0.3)$ & $(0.7,0.4)$ & $(0.4,0.3)$ & $(0.9,0.3)$ & $(0.4,0.4)$ \\
$\widetilde{\mathcal{Q}}_{u_{2}}^{\beta}$ & $(0.6,0.5)$ & $(0.7,0.8)$ & $(0.6,0.5)$ & $(0.6,0.4)$ & $(0.6,0.8)$ \\
$\widetilde{\mathcal{Q}}_{u_{3}}^{\beta}$ & $(0.8,0.7)$ & $(0.4,0.2)$ & $(0.8,0.4)$ & $(0.8,0.7)$ & $(0.7,0.4)$ \\
$\widetilde{\mathcal{Q}}_{u_{4}}^{\beta}$ & $(0.7,0.4)$ & $(0.6,0.5)$ & $(0.4,0.4)$ & $(0.8,0.3)$ & $(0.4,0.5)$ \\
$\widetilde{\mathcal{Q}}_{u_{5}}^{\beta}$ & $(0.5,0.3)$ & $(0.59,0.4)$ & $(0.7,0.38)$ & $(0.5,0.2)$ & $(0.58,0.4)$ \\
\hline
\end{tabular}

Table 17 Result for $\widetilde{\mathcal{Q}}_{u_{k}}^{(0 . \hat{8}, 0.4)}, k=1,2, \ldots, 5$

\begin{tabular}{llllll}
\hline & $u_{1}$ & $u_{2}$ & $u_{3}$ & $u_{4}$ & $u_{5}$ \\
\hline$\hat{\widetilde{\mathcal{Q}}}_{u_{1}}^{\beta}$ & $(0.3,0.8)$ & $(0.4,0.7)$ & $(0.3,0.4)$ & $(0.3,0.9)$ & $(0.4,0.4)$ \\
$\tilde{\widetilde{\mathcal{Q}}}_{u_{2}}^{\beta}$ & $(0.5,0.6)$ & $(0.8,0.7)$ & $(0.5,0.6)$ & $(0.4,0.6)$ & $(0.8,0.6)$ \\
$\hat{\widetilde{\mathcal{Q}}}_{u_{3}}^{\beta}$ & $(0.7,0.8)$ & $(0.2,0.4)$ & $(0.4,0.8)$ & $(0.7,0.8)$ & $(0.4,0.7)$ \\
$\hat{\widetilde{\mathcal{Q}}}_{u_{4}}^{\beta}$ & $(0.4,0.7)$ & $(0.5,0.6)$ & $(0.4,0.4)$ & $(0.3,0.8)$ & $(0.5,0.4)$ \\
$\tilde{\widetilde{\mathcal{Q}}}_{u_{5}}^{\beta}$ & $(0.3,0.5)$ & $(0.4,0.59)$ & $(0.38,0.7)$ & $(0.2,0.7)$ & $(0.4,0.58)$ \\
\hline
\end{tabular}

Table 18 Result for ${ }_{2} \widetilde{\mathbb{Q}}_{u_{k}}^{\beta}, k=1,2, \ldots, 5$

\begin{tabular}{llllll}
\hline & $u_{1}$ & $u_{2}$ & $u_{3}$ & $u_{4}$ & $u_{5}$ \\
\hline${ }_{2} \widetilde{\mathbb{Q}}_{u_{1}}^{\beta}$ & $(0.8,0.3)$ & $(0.7,0.4)$ & $(0.4,0.3)$ & $(0.9,0.3)$ & $(0.4,0.4)$ \\
$2 \widetilde{\mathbb{Q}}_{u_{2}}^{\beta}$ & $(0.6,0.5)$ & $(0.8,0.7)$ & $(0.6,0.5)$ & $(0.6,0.4)$ & $(0.8,0.6)$ \\
$2 \widetilde{\mathbb{Q}}_{u_{3}}^{\beta}$ & $(0.8,0.7)$ & $(0.4,0.2)$ & $(0.8,0.4)$ & $(0.8,0.7)$ & $(0.7,0.4)$ \\
$2 \widetilde{\mathbb{Q}}_{u_{4}}^{\beta}$ & $(0.7,0.4)$ & $(0.6,0.5)$ & $(0.4,0.4)$ & $(0.8,0.3)$ & $(0.5,0.4)$ \\
$2 \widetilde{\mathbb{Q}}_{u_{5}}^{\beta}$ & $(0.5,0.3)$ & $(0.59,0.4)$ & $(0.7,0.38)$ & $(0.7,0.2)$ & $(0.58,0.4)$ \\
\hline
\end{tabular}

Step 4: The lower and upper approximation of $\mathscr{D}$ using 3 -q-ROF $\beta$ CRSs are calculated as the following.

First, we investigate the q-ROF $\beta$-neighborhood and qROF complementary $\beta$-neighborhood as established, respectively, in Tables 16 and 17.

Now, we can calculate ${ }_{2} \widetilde{\mathbb{Q}}_{u}^{\beta}$ as the following Table 18 .

$$
\begin{gathered}
\mathcal{L}_{4}^{\mathrm{q}-\mathrm{ROF}}(\mathscr{D})=\left\{\begin{array}{c}
\left(u_{1}, 0.12943,0.4\right),\left(u_{2}, 0.12943,0.7\right), \\
\left(u_{3}, 0.12943,0.7\right),\left(u_{4}, 0.12943,0.5\right), \\
\left(u_{5}, 0.12943,0.4\right)
\end{array}\right\}, \\
\mathcal{U}_{4}^{\mathrm{q}-\mathrm{ROF}}(\mathscr{D})=\left\{\begin{array}{c}
\left(u_{1}, 0.0 .9,0.10907\right),\left(u_{2}, 0.8,0.10907\right), \\
\left(u_{3}, 0.8,0.10907\right),\left(u_{4}, 0.8,0.10907\right), \\
\left(u_{5}, 0.7,0.10907\right)
\end{array}\right\}
\end{gathered}
$$

Step 4: Compute the sorting function of the MAqROFDM problem as follows.

$$
\begin{gathered}
\varphi\left(u_{1}\right)=0.0538, \quad \varphi\left(u_{2}\right)=0.0765, \quad \varphi\left(u_{3}\right)=0.0765 \\
\varphi\left(u_{4}\right)=0.0585, \quad \varphi\left(u_{5}\right)=0.0473
\end{gathered}
$$

Then the order of these candidates is $u_{2} \approx u_{3}>u_{4}>u_{1}>$ $u_{5}$, thus the second candidate is proper for this job.

\section{Comparative analysis}

The goal of this part is to explain the differences between our proposed study and the previous work. We split our vision into two parts, that is, CPFRS and Cq-ROFRS, respectively.

(1) The prime objective of the given method of CPFRS is capable of promoting the lower approximation and minimizing the upper approximation of the former investigation by Zhan's in [52] as apparent in Examples 2, 5 and 8 . To state the rapprochements through different processes, that is Yager's process [45], Zhang's process [49], Zhan's process [52] and our process, the classification score of these decision-making samples are recorded in Table 19 and 20 . Also, we demonstrate Fig. 1, to show the values of ordering variables between Zhan's model and our model. From this figure, you can see that our outcomes are greater than Zhan's outcomes, and also the first candidate is the suitable one among all in the two presented models.

Tables 19 and 20 interpreted that the optimal decision is the same alternative $u_{1}$ for the four processes i.e., 
Table 19 Result for scores by PF $\beta$ CAS

Table 20 Result for scores by PF $\beta$ CAS

\begin{tabular}{lllllllll}
\hline Different processes & \multicolumn{2}{l}{ Obtain a value } \\
\cline { 2 - 8 } & $u_{1}$ & $u_{2}$ & $u_{3}$ & $u_{4}$ & $u_{5}$ & $u_{6}$ & $u_{7}$ & $u_{8}$ \\
\hline Zhan's model [52] & 0.2029 & 0.124 & 0.1211 & 0.123 & 0.1316 & 0.1575 & 0.1259 & 0.0644 \\
Our model & 0.2486 & 0.2354 & 0.2479 & 0.202 & 0.2354 & 0.209 & 0.2347 & 0.1745 \\
\hline
\end{tabular}

Different processes

Yager's process [45]

Zhang's process [49]

Zhan's process [52]

Our process
Obtain a decision

$u_{1} \geq u_{5} \geq u_{6} \geq u_{7} \geq u_{8} \geq u_{4} \geq u_{2} \geq u_{3}$

$u_{1} \geq u_{5} \geq u_{7} \geq u_{6} \geq u_{8} \geq u_{4} \geq u_{2} \geq u_{3}$

$u_{1} \geq u_{6} \geq u_{5} \geq u_{7} \geq u_{2} \geq u_{4} \geq u_{3} \geq u_{8}$

$u_{1} \geq u_{3} \geq u_{2} \approx u_{5} \geq u_{7} \geq u_{6} \geq u_{4} \geq u_{8}$

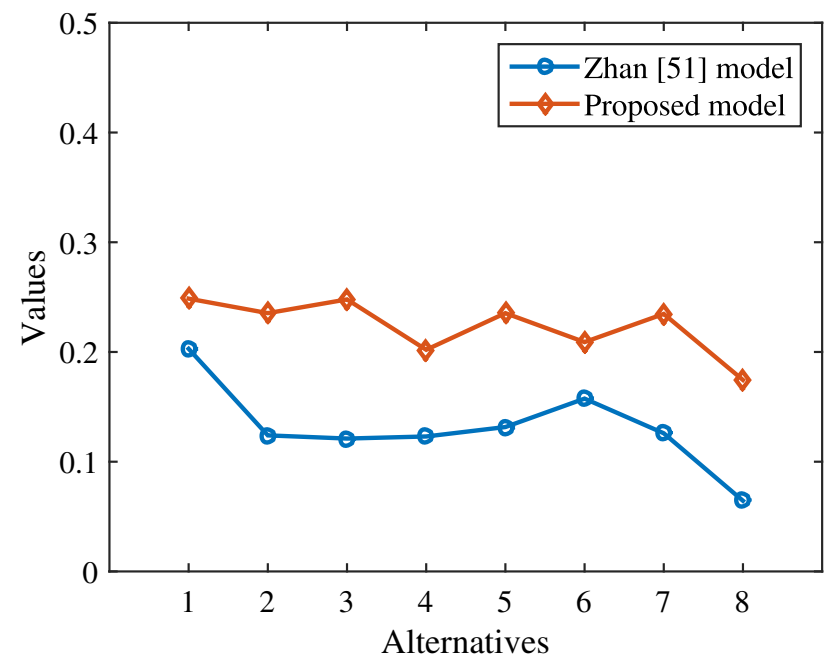

Fig. 1 Representations of the ordering alternatives

(Yager's [45], Zhang's [48], Zhan's [52] and our's), that is make our approach is feasible and effective.

Figures 2 and 3 states the another way to show that the variances through Zhan's method [52] and our method. Figure 2 contains two parts. The left part illustrates that our membership $\vartheta$ of the lower approximation is higher than in Zhan et al. [52]. On the other hand, the right part shows that our non-membership $\zeta$ of the lower approximation is lower than in Zhan et al. [52]. This means that our lower is better than Zhan's lower from the view of raising the lower approximation which makes our approach is suitable than others.

Figure 3 also have two figures. The left one explained that the our membership $\vartheta$ of the upper approximation is lower than in Zhan et al. [52]. In contrast, the other figure clarifies that the our non- membership $\zeta$ of the upper approximation is higher than in Zhan et al. [52]. This shows that our upper is better than Zhan's upper from the view of lowering the upper approximation that makes our model is more appropriate than others.

To sum, these two images mean that our lower approximation is better than Zhan-lower and our upper approximation is lower than Zhan-upper which makes our proposed study is more appropriate than others. Therefore, the presented method is reliable, and effective and is considered as a generalization of the Zhan's method.

(2) $\mathrm{q}-\mathrm{ROF}$ is considered as the generalization of PFS (where $\mathrm{q}=2$ ) and IFS (where $\mathrm{q}=1$ ). The presented model of Cq-ROFRS is the natural extension to Hussain et al. [57] which investigates the novel covering method under the
Fig. 2 Representations of the lower approximations between methods in [52] and Definition 19
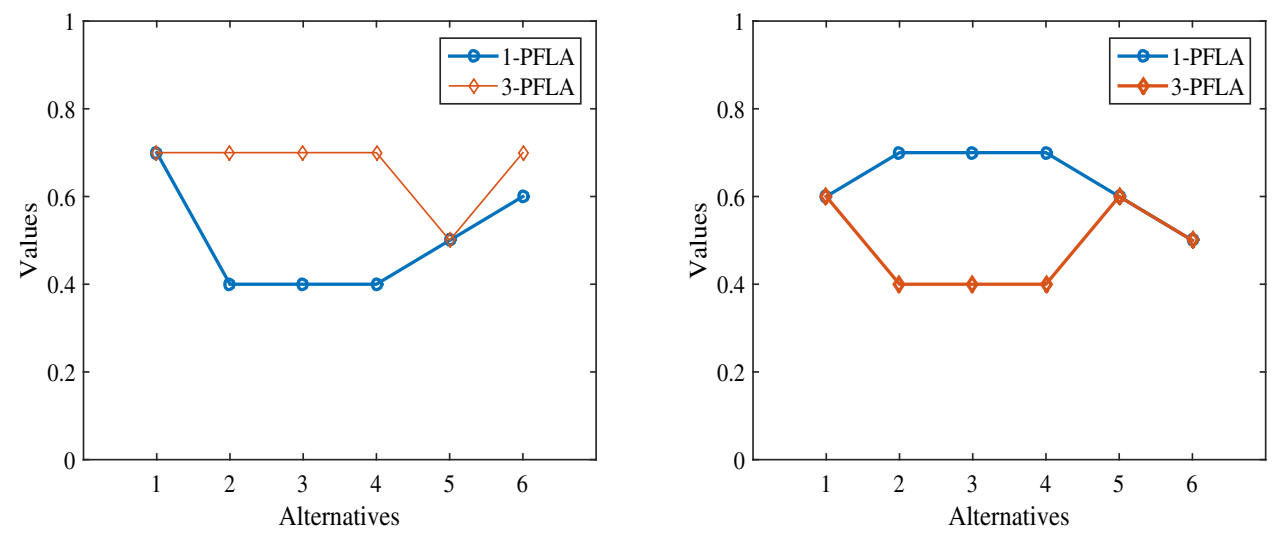
Fig. 3 Representations of the upper approximations between methods in [52] and

Definition 19

Table 21 Result for orders by q-ROF $\beta$ CAS
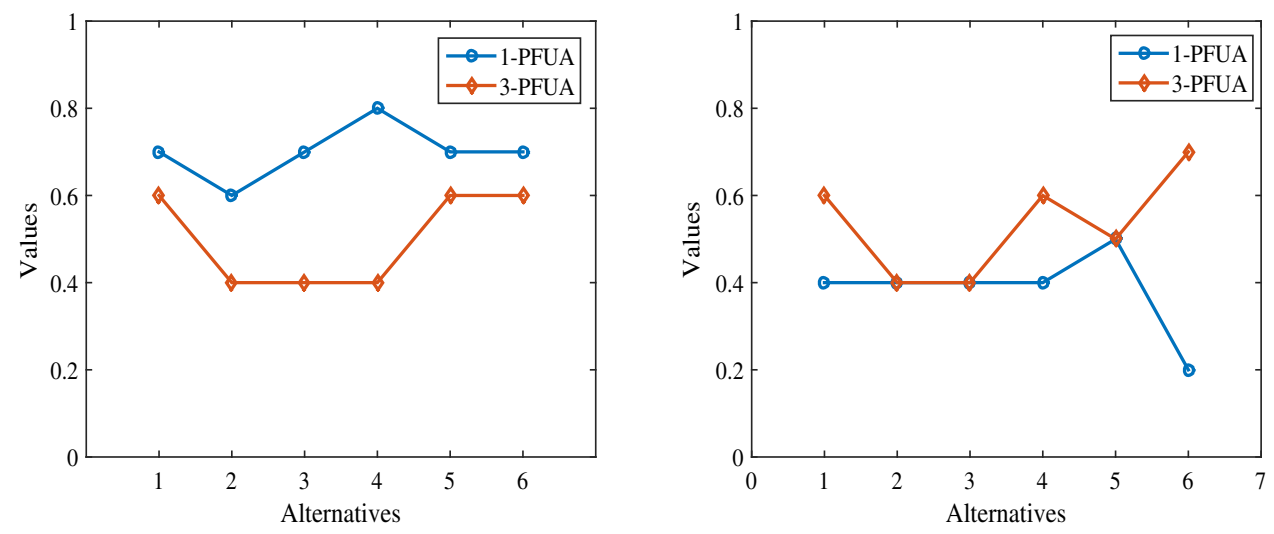

\begin{tabular}{llllll}
\hline Distinct models & \multicolumn{2}{l}{ Obtain a result } & & & \\
\cline { 2 - 5 } & $u_{1}$ & $u_{2}$ & $u_{3}$ & $u_{5}$ & 0.0585 \\
\hline Hussain's model [57] & 0.0538 & 0.0842 & 0.0765 & 0.0573 \\
Our model & 0.0538 & 0.0765 & 0.0765 & 0.0585 & 0.0473 \\
\hline
\end{tabular}

Table 22 Result for orders by q-ROF $\beta$ CAS

\begin{tabular}{ll}
\hline Distinct models & Obtain a decision \\
\hline Hussain model [57] & $u_{2}>u_{3}>u_{4}>u_{1}>u_{5}$ \\
Our model & $u_{2} \approx u_{3}>u_{4}>u_{1}>u_{5}$ \\
\hline
\end{tabular}

notion of $\mathrm{q}$-ROF $\beta$-neighborhoods. Here, we present the definition of $\mathrm{q}-\mathrm{ROF}$ complementary $\beta$-neighborhoods and combine these two types of neighborhoods to investigate two other kinds of q-ROF $\beta$-neighborhoods. Hence, we used these types to construct three novel kinds of CqROFRS model. Now, we build the Tables 21 and 22 to demonstrate the outcomes between Hussain et al. [57] and our's.

From Tables 21 and 22, we can say that the best decree is the second candidate $u_{2}$ among two different approaches (i.e.,
Hussain et al. [57] and our's). This means that the decision is the same alternative. This proofs that the proposed model is effective and reliable.

Figures 4 and 5 states the another way to show that the variances through Hussain's method [52] and our method.

Figure 4 splits into two parts. The left split illustrates that the $\mu$ of the lower approximation is higher than in Hussain et al. [57]. On the other hand, the right split shows that the $v$ of the lower approximation is lower than in Hussain et al. [57].

Figure 5 also have two images. These figures explained the differences between $\mu$ and $v$ in Hussain et al. [57] and ours.

In particular, the above two images mean that our lower approximation is better than Hussain-lower and our upper approximation is lower than Hussain-upper. Therefore, the presented method is reliable, and effective and is considered as a generalization of the Hussain method.
Fig. 4 Representations of the lower approximations between methods in [57] and Definition 31
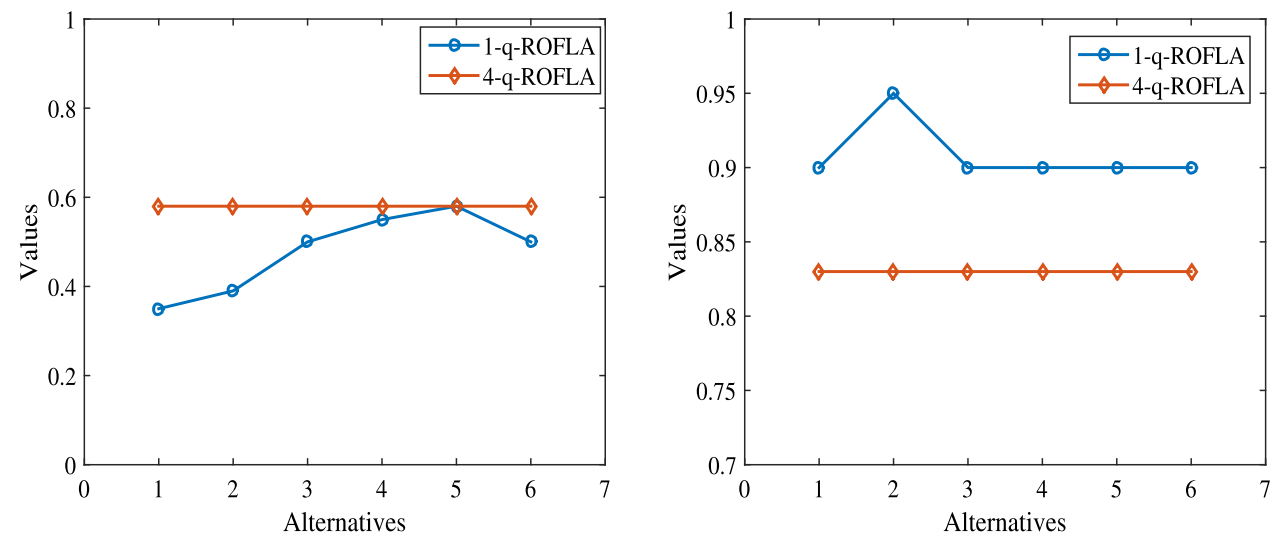
Fig. 5 Representations of the upper approximations between methods in [57] and Definition 31
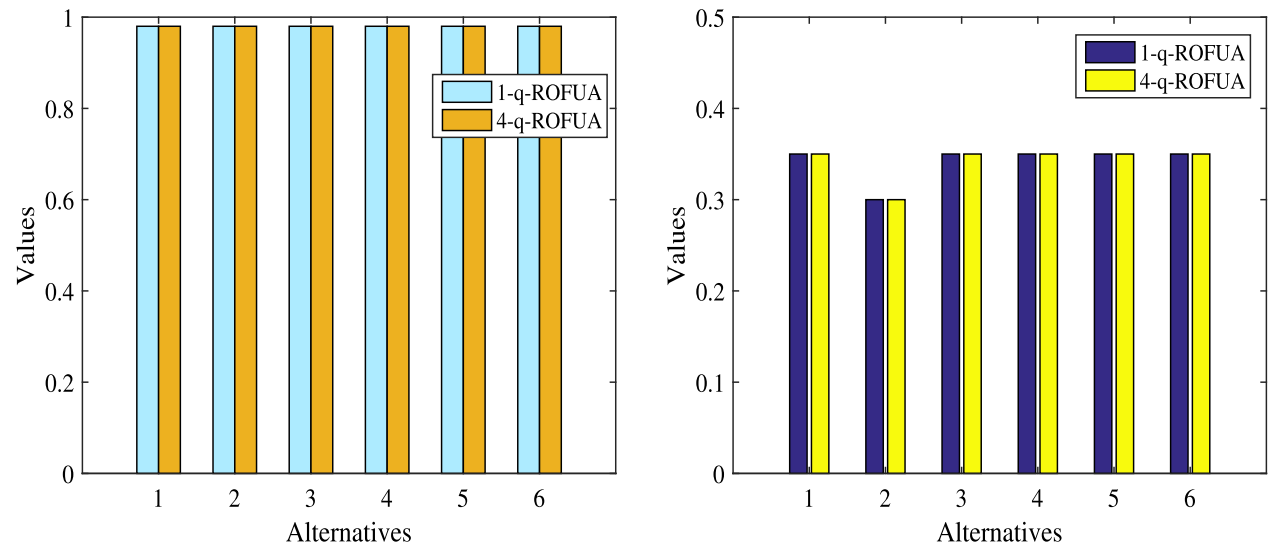

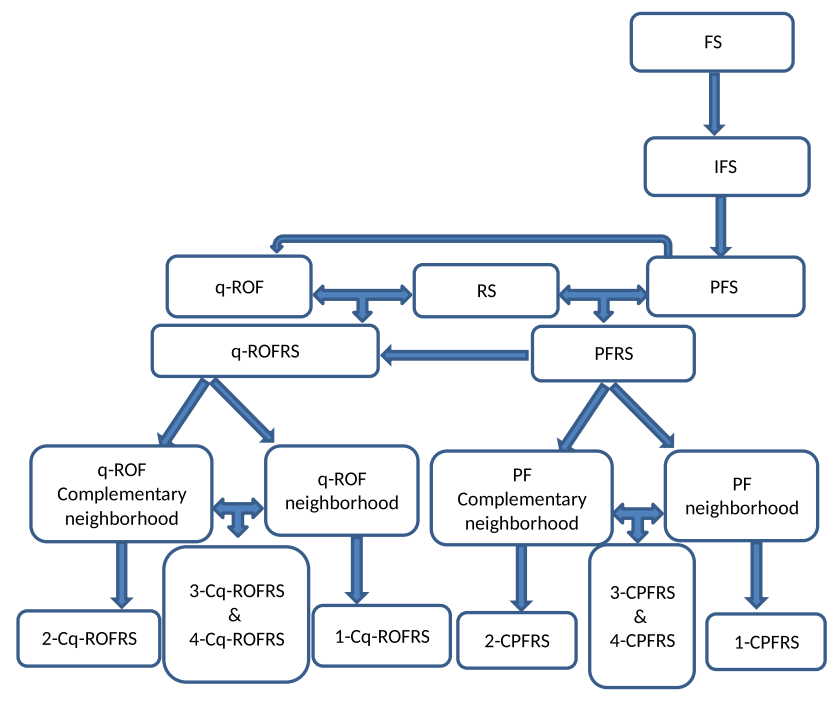

Fig. 6 The organization of the proposed methods

To simplify our studies and the relations between our presented models in CPFRS and Cq-ROFRS, we give the following Fig. 6 that explained in briefly our vision in this study. This Figure clarifies that the Cq-ROFRS is a generalization of CPFRS.

\section{Conclusion}

The main purpose of the proposed article is to improve Zhan et al's model in [52] and Hussain et al.'s model in [57]. The chief investigation of the study is summarized as follows.

(1) We extend the study of CPFRS through PF complementary $\beta$-neighborhood. By joining the concept of PF $\beta$ neighborhood and PF complementary $\beta$-neighborhood, we obtain three novel methods on a CPFRS. Also, we investigate the comparisons between the Zhan et al.'s process and our process. These differences illustrate that $3-\mathrm{PF} \beta \mathrm{CRS}$ is the best approximations among $1-\mathrm{PF} \beta \mathrm{CRS}$
(Zhan et al.'s model), 2-PF $\beta$ CRS and 4-PF $\beta$ CRS. It is easy to see Tables 19 and 20 and Figs. 1, 2 and 3, which shows these comparisons clearly.

(2) To generalize Hussain et al.'s model and our models via $\mathrm{PF}$ in "PF complementary $\beta$-neighborhood and three novel kinds of CPFRS", the meaning of q-ROF complementary $\beta$-neighborhood are distinguished. Also, we combine the q-ROF $\beta$-neighborhood and q-ROF complementary $\beta$-neighborhood to estimate new models of Cq-ROFRS. Moreover, we discuss the differences between Hussain et al.'s method and our's. These comparisons explain that 4-q-ROF $\beta$ CRS is the best approximations among 1-q-ROF $\beta$ CRS (Hussain et al.'s model), $2-\mathrm{q}-\mathrm{ROF} \beta \mathrm{CRS}$ and 3-q-ROF $\beta$ CRS. It is handy to see Tables 15 and 16 and Figs. 4 and 5, which shows these differences clearly.

(3) In short, the proposed models are extended on the first studies on CPFRS by Zhan et al's [52] and Cq-ROFRS by Hussain et al.'s [57] methods. Zhan et al.'s paper is a generalization to the notions on covering method by IFS and Hussain et al.'s article is a generalization to the last studies on CPFRS by Zhan et al.'s, so it is already generalized to IFS. This article is working in the same direction as these studies and is splits into two main parts. The first one talks about the CPFRS model and makes a generalization of Zhan et al.'s model. The second part presents the new generalization of Zhan et al.'s model, Hussain et al.'s model, and our's in the first part by the methodology of a Cq-ROFRS model.

(4) Cq-ROFRS investigates to solve the limitations in the CPFRS, CIFRS, and CFRS. CIFRS deals with the membership and non-membership degrees not only on the membership degree as CFRS. CPFRS is more accurate than CIFRS because it deals with membership and nonmembership degrees that their square sum is less than or equal to 1 . To treat the limitation on CPFRS, Cq-ROFRS is considered as a generalization of CPFRS, CIFRS, and CFRS because of the values of $q$. Further studies will 
focus on explaining the topological properties of the CPFRS and Cq-ROFRS as in [58,59] and extend to the graph theory as in [60].

\begin{abstract}
Author Contributions HG: Methodology; Validation; Formal analysis; Data curation; Writing—original draft; Writing_review \& editing; Visualization. MA: Methodology; Validation; Formal analysis; Data curation; Writing - original draft.
\end{abstract}

Funding There is no external funding.

Data availability All data generated or analyzed during this study appear in the submitted article.

\section{Declarations}

Conflict of interest The authors declare no conflict of interest.

Ethical statement This manuscript is the authors' original work and has not been published nor has it been submitted simultaneously elsewhere.

Open Access This article is licensed under a Creative Commons Attribution 4.0 International License, which permits use, sharing, adaptation, distribution and reproduction in any medium or format, as long as you give appropriate credit to the original author(s) and the source, provide a link to the Creative Commons licence, and indicate if changes were made. The images or other third party material in this article are included in the article's Creative Commons licence, unless indicated otherwise in a credit line to the material. If material is not included in the article's Creative Commons licence and your intended use is not permitted by statutory regulation or exceeds the permitted use, you will need to obtain permission directly from the copyright holder. To view a copy of this licence, visit http://creativecomm ons.org/licenses/by/4.0/.

\section{References}

1. Pawlak Z (1982) Rough sets. Int J Comput Inf Sci 11(5):341-356

2. Pawlak Z (1985) Rough concept analysis. Bull Pol Acad Sci Math 33:9-10

3. Atef M, Khalil AM, Li SG, Azzam A, El Atik AA (2020) Comparison of six types of rough approximations based on $\mathrm{j}$-neighborhood space and j-adhesion neighborhood space. J Intell Fuzzy Syst 39:4515-4531

4. El Atik AA, Nawar AS, Atef M (2021) Rough approximation models via graphs based on neighborhood systems. Granul Comput 6:1025-1035. https://doi.org/10.1007/s41066-020-00245-z

5. Hu Q, Zhang L, Chen D, Pedrycz W, Yu D (2010) Gaussian kernel based fuzzy rough sets: model, uncertainty measures and applications. Int J Approx Reason 51(4):453-471

6. Jensen R, Shen Q (2004) Semantics-preserving dimensionality reduction: rough and fuzzy-rough-based approaches. IEEE Trans Knowl Data Eng 16(12):1457-1471

7. Liu G, Zhu W (2008) The algebraic structures of generalized rough set theory. Inf Sci 178:4105-4113

8. Pal S, Mitra P (2004) Case generation using rough sets with fuzzy representation. IEEE Trans Knowl Data Eng 16:293-300
9. Qian Y, Liang J, Dang C (2009) Knowledge structure, knowledge granulation and knowledge distance in a knowledge base. Int $\mathrm{J}$ Approx Reason 50(1):174-188

10. Yang XP, Li TJ (2006) The minimization of axiom sets characterizing generalized approximation operators. Inf Sci 176:887-899

11. Yao YY (2010) Three-way decisions with probabilistic rough sets. Inf Sci 180(3):341-353

12. Zhang H, Liang H, Liu D (2004) Two new operators in rough set theory with applications to fuzzy sets. Inf Sci 166(1-4):147-165

13. Ziarko W (1993) Variable precision rough set model. J Comput Syst Sci 46(1):39-59

14. Pomykala JA (1987) Approximation operations in approximation space. Bull Pol Acad Sci 35:653-662

15. Pomykala JA (1988) On definability in the nondeterministic information system. Bull Pol Acad Sci 36:193-210

16. Yao YY (1998) Relational interpretations of neighborhood operators and rough set approximation operators. Inf Sci 111:239-259

17. Yao YY, Yao B (2012) Covering based rough set approximations. Inf Sci 200:91-107

18. Couso I, Dubois D (2011) Rough sets, coverings and incomplete information. Fund Inf 108(3-4):223-247

19. Bonikowski Z, Bryniarski E, Wybraniec-Skardowska U (1998) Extensions and intentions in rough set theory. Inf Sci 107:149-167

20. Zhu W (2007) Topological approaches to covering rough sets. Inf Sci 177:1499-1508

21. Zhu W, Wang F (2003) Reduction and axiomization of covering generalized rough sets. Inf Sci 152:217-230

22. Zhu W, Wang F (2007) On three types of covering rough sets. IEEE Trans Knowl Data Eng 19:1131-1144

23. Zhu W, Wang F (2012) The fourth types of covering-based rough sets. Inf Sci 201:80-92

24. Tsang ECC, Chen D, Yeung DS (2008) Approximations and reducts with covering generalized rough sets. Comput Math Appl $56: 279-289$

25. Xu WH, Zhang WX (2007) Measuring roughness of generalized rough sets induced a covering. Fuzzy Sets Syst 158:2443-2455

26. Liu G, Sai Y (2009) A comparison of two types of rough sets induced by coverings. Int J Approx Reason 50:521-528

27. Ma L (2012) On some types of neighborhood related covering rough sets. Int J Approx Reason 53:901-911

28. Dubois D, Prade H (1990) Rough fuzzy sets and fuzzy rough sets. Int J Gen Syst 17:191-201

29. Deng T, Chen Y, Xu W, Dai Q (2007) A novel approach to fuzzy rough sets based on a fuzzy covering. Inf Sci 177:2308-2326

30. Atef M, Nada S, Gumaei A, Nawar AS (2021) On three types of soft rough covering-based fuzzy sets. J Math. https://doi.org/10. 1155/2021/6677298 (Article ID 6677298)

31. Atef M, Nada SI (2021) On three types of soft fuzzy coverings based rough sets. Math Comput Simul 185:452-467

32. Li TJ, Leung Y, Zhang WX (2008) Generalized fuzzy rough approximation operators based on fuzzy covering. Int J Approx Reason 48:836-856

33. Ma J, Atef M, Nada S, Nawar A (2020) Certain types of coveringbased multigranulation $(\mathscr{I}, \mathscr{T})$-fuzzy rough sets with application to decision-making. Complexity 2020:1-20. https://doi.org/10. $1155 / 2020 / 6661782$

34. Ma J, Atef M, Khalil AM, Hassan N, Chen G-X (2020) Novel models of fuzzy rough coverings based on fuzzy $\alpha$-neighborhood and its application to decision-making. IEEE Access 8:224354224364. https://doi.org/10.1109/ACCESS.2020.3044213

35. Ma L (2016) Two fuzzy covering rough set models and their generalizations over fuzzy lattices. Fuzzy Sets Syst 294:1-17

36. Yang B, Hu BQ (2017) On some types of fuzzy covering based rough sets. Fuzzy Sets Syst 312:36-65

37. Yang B, Hu BQ (2019) Fuzzy neighborhood operators and derived fuzzy coverings. Fuzzy Sets Syst 370:1-33 
38. Zadeh LA (1965) Fuzzy sets. Inf Control 8:338-353

39. Atanassov KT (1986) Intuitionistic fuzzy sets. Fuzzy Sets Syst 20:87-96

40. Atanassov KT, Pasi G, Yager RR (2015) Intuitionistic fuzzy interpretations of multi-criteria multi-person and multi-measurement tool decision-making. Int J Syst Sci 36:859-868

41. Huang B, Guo C, Zhang Y, Li H, Zhou X (2014) Intuitionistic fuzzy multigranulation rough sets. Inf Sci 277:299-320

42. Huang B, Guo C, Zhang Y, Li H, Zhou X (2016) An intuitionistic fuzzy graded covering rough sets. Knowl-Based Syst 107:155-178

43. Alcantud JCR, Torra V (2018) Decomposition theorems and extension principles for hesitant fuzzy sets. Inf Fusion 41:48-56

44. Yager RR (2013) Pythagorean fuzzy subsets. In: Joint IFSA world congress and NAFIPS annual meeting (IFSA/NAFIPS), Edmonton, AB, pp 57-61. https://doi.org/10.1109/IFSA-NAFIPS.2013. 6608375

45. Yager RR (2014) Pythagorean membership grades in multi-criteria decision making. IEEE Trans Fuzzy Syst 22:958-965

46. Yager RR (2016) Properties and applications of Pythagorean fuzzy sets. Springer, Berlin

47. Garg H (2016) A new generalized Pythagorean fuzzy information aggregation using Einstein operations and its application to decision making. Int J Intell Syst 31:886-920

48. Garg H (2017) Generalized Pythagorean geometric aggregation operations using Einstein t-norm for multicriteria decision-making problem. Int J Intell Syst 32:597-630

49. Zhang XL, Xu ZS (2014) Extensions of TOPSIS for multiple criteria decision making with Pythagorean fuzzy sets. Int J Intell Syst 29:1061-1078

50. Hussain A, Ali MI, Mahmood T (2020) Pythagorean fuzzy soft rough sets and their applications in decision-making. J Taibah Univ Sci14(1):101-113
51. Wang L, Garg H (2021) Algorithm for multiple attribute decisionmaking with interactive archimedean norm operations under Pythagorean fuzzy uncertainty. Int J Comput Intell Syst 14(1):503527

52. Zhan J, Sun B, Zhang X (2020) PF-TOPSIS method based on CPFRS models: an application to unconventional emergency events. Comput Ind Eng 139:106192

53. Yager RR (2017) Generalized orthopair fuzzy sets. IEEE Trans Fuzzy Syst 25(5):1222-1230

54. Yager RR, Alajlan N (2017) Approximate reasoning with generalized orthopair fuzzy sets. Inf Fusion 38:65-73

55. Ali MI (2019) Another view on q-rung orthopair fuzzy sets. Int $\mathbf{J}$ Intell Syst 33:2139-2153

56. Garg H (2021) CN-q-ROFS: connection number based q-rung orthopair fuzzy set and their application to decision-making process. Int J Intell Syst 36(7):2106-2143

57. Hussain A, Ali AI, Mahmood T (2019) Covering based q-rung orthopair fuzzy rough set model hybrid with TOPSIS for multiattribute decision making. J Intell Fuzzy Syst 37:981-993

58. Olgun M, Unver M, Yardimci S (2021) Pythagorean fuzzy points and applications in pattern recognition and Pythagorean fuzzy topologies. Soft Comput 25(7):5225-5232

59. Turkarslan E, Unver M, Olgun M (2021) $\boldsymbol{q}$-Rung orthopair fuzzy topological spaces. Lobachevskii J Math 42(2):470-478

60. Atef M, El Atik AA, Nawar A (2021) Fuzzy topological structures via fuzzy graphs and their applications. Soft Comput 25(8):60136027

Publisher's Note Springer Nature remains neutral with regard to jurisdictional claims in published maps and institutional affiliations. 